\title{
A STUDY OF ASSOCIATION IN INSANITY.
}

By GRACE HELEN KENT, A. M., AND A. J. ROSANOFF, M. D., Kings Park State Hospital, N. Y.

\section{PART II. ASSOCIATION IN INSANE SUBJECTS.}

§ I. General Survey of Pathological Material.

The pathological material which forms the basis of the present part of our study consists mainly of two hundred and forty-seven test records obtained for the most part from patients at the Kings Park State Hospital.

The different groups from which the cases were selected, together with the number from each group, are shown in Table I.

TABLE I.

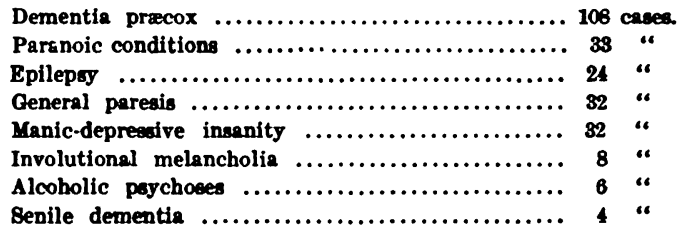

A comparison of our pathological with our normal material en masse reveals in the former evidence of a weakening of the normal tendency to respond by common reactions. This is shown in Table II.

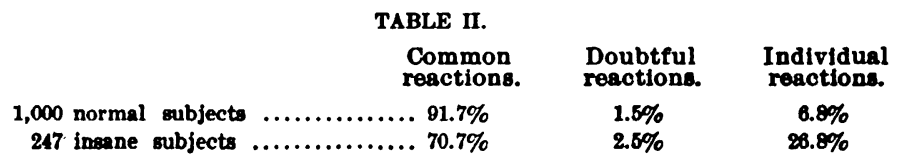

It seems evident from this that pathological significance attaches mainly to individual reactions, so that our study resolves itself largely into ( $\mathrm{I}$ ) an analysis and classification of individual reactions and (2) an attempt to determine what relationship, if any, exists between the different types of reactions and the different clinical forms of mental disease. 


\section{§ 2. Classification of Reactions.}

Those who have attempted to use the association test in the study of insanity have felt the need of a practical classification of reactions, and have at the same time encountered the difficulty of establishing definite criteria for distinguishing the different groups from one another. It is a comparatively simple matter to make these distinctions in a general way and even to formulate a more or less comprehensive theoretical classification, but there still remains much difficulty in practice. We have made repeated attempts to utilize various systems of classification which involve free play of personal equation in their application. Although for us the matter is greatly simplified by the elimination of all the common reactions with the aid of the frequency tables, we have nevertheless met with no success. The distinctions made by either of us have on no occasion fully satisfied, at the second reading, either the one who made them or the other, while a comparison of the distinctions made by each of us independently has shown a disagreement to the extent of 20-35 per cent.

We sought, therefore, to formulate a classification in which the various groups should be so defined as to obviate the interference of personal equation in the work of applying it, hoping thus to achieve greater accuracy. In this we can lay claim to only partial success; for, in the first place, having satisfactorily defined a number of groups, we found it necessary in the end to provide a special group for unclassified reactions, into which falls more than one-third of the total number of individual reactions; and, in the second place, in at least two of our groups the play of personal equation has not been entirely eliminated, so that there is still a possibility of error to the extent of five per cent of individual reactions, which means approximately one per cent of the total number of reactions. We have found, however, that in spite of these shortcomings the classification here proposed is more serviceable than others which, though more comprehensive, are at the same time lacking in definiteness.

Our classification consists of the following classes, groups and subdivisions : 
I. Common reactions.

I. Specific reactions.

2. Non-specific reactions.

II. Doubtful reactions.

III. Individual reactions.

I. Normal reactions.

2. Pathological reactions:

A. Derivatives of stimulus words.

B. Partial dissociation:

(a) Non-specific reactions.

(b) Sound reactions:

a. Words.

B. Neologisms.

(c) Word complements.

(d) Particles of speech.

C. Complete dissociation :

(a) Perseveration:

a. Association to preceding stimulus.

$\beta$. Association to preceding reaction.

$\boldsymbol{\gamma}$. Repetition of preceding stimulus.

o. Repetition of previous stimulus.

e. Repetition of preceding reaction.

५. Repetition of previous reaction.

$\eta$. Reaction repeated five times (stereotypy).

(b) Neologisms without sound relation.

3. Unclassified.

\section{§ 3. Non-Specific Reactions; Doubtful Reactions.}

Non-specific Reactions.-It has already been intimated that common reactions are in the vast majority of instances to be regarded as normal. From amongst them, however, a fairly definite group can be separated out which seems to possess some pathological significance, namely, the group which we have termed non-specific.

In this group are placed words which are so widely applicable as to serve as more or less appropriate reactions to almost any of our stimulus words. That such reactions are in value inferior to the remaining group of common reactions, which we have termed, in contradistinction, specific reactions, is perhaps sufficiently obvious; we shall speak later, however, of their occurrence in both normal and insane cases. 
It is not always easy to judge whether or not a given reaction should be classed as non-specific. A study of our material made with special reference to this type of reactions has enabled us to select the following list of words, any of which, occurring in response to any stimulus word, is classed as a non-specific reaction :

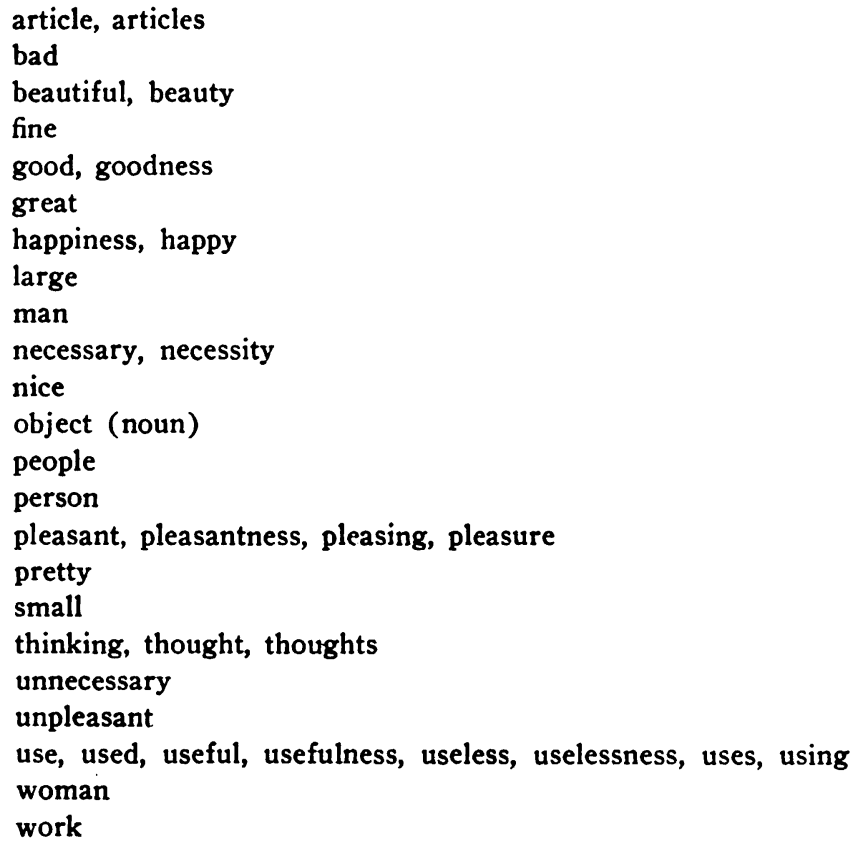

It should be mentioned that some of these words occur as reactions to one or several stimulus words with such frequency (citi$z e n-\operatorname{man}$, value 27.8 per cent; health-good, value 9.4 per cent) as to acquire in such instances a value as high as that of strictly specific reactions.

Doubtful Reactions have already been defined (p. 40) : any reaction word which is not found in the table in its identical form, but which is a grammatical variant or derivative of a word found there, is placed in this group. 


\section{§ 4. Individual Reactions; Explanation of Groups AND Methods of Application.}

Normal Reactions.-Inasmuch as the frequency tables do not exhaust all normal possibilities of reaction, a certain number of reactions which are essentially normal are to be found among the individual reactions. In order to separate these from the pathological reactions, we have compiled an appendix to the frequency tables, consisting mainly of specific definitions of groups of words to be included under each stimulus word in our list. This appendix will be found at the end of this paper.

A word of explanation is perhaps due as to the manner in which the appendix has been compiled. It was developed in a purely empirical way, the basis being such individual reactions, given by both normal and insane subjects, as seemed in our judgment to be obviously normal.

It must be acknowledged that the appendix falls short of all that might be desired. In the first place, its use involves to some slight extent the play of personal equation, and it therefore constitutes a source of error; in the second place, it is in some respects too inclusive while in other respects it is not sufficiently so. However, the error due to personal equation is slight; the inclusion of certain "far-fetched" or even frankly pathological reactions may be discounted by bearing in mind that the general value of this group is not equal to that of the group of common reactions; and the number of strictly normal reactions which are not included is after all small. Our experience has shown us that the appendix constitutes an important aid in the analysis of individual reactions.

Pathological Reactions. Derivatives of Stimulus Words.-We place here any reaction which is a grammatical variant or derivative of a stimulus word. The tendency to give such reactions seems to be dependent upon a suspension or inhibition of the normal process by which the stimulus word excites the production of a new concept, for we have here not a production of a new concept but a mere change in the form of the stimulus word. As examples of such reactions may be mentioned: eating-eatables, short-shortness, sweet-sweetened, quiet-quietness.

Partial Dissociation.-We have employed the term dissociation to indicate a rupture of that bond-whatever be its nature- 
which may be supposed to exist normally between stimulus and reaction and which causes normal persons to respond in the majority of instances by common reactions. And we speak of partial dissociation where there is still an obvious, though weak and superficial, connection. Under this heading we can differentiate four types:

Non-specific Beactions have already been defined; we distinguish those in this class from those in the class of common reactions by means of the frequency tables.

Sound Reactions.-This type requires no explanation; the main difficulty is to decide what degree of sound similarity between stimulus and reaction should be deemed sufficient for placing a reaction under this heading. The total number of different sounds used in language articulation is, of course, small, so that any two words are liable to present considerable chance similarity. Some time ago we estimated the average degree of sound similarity between stimulus words and reaction words in a series of one hundred test records obtained from normal persons; we found that on the average 14.53 per cent of the sounds of the stimulus words were reproduced, in the same order, in the reaction word. Our experience finally led us to adopt the following general rule: A reaction is to be placed under this heading when fifty per cent of the sounds of the shorter word of the pair are identical with sounds of the longer word and are ranged in the same order.

Among sound reactions we occasionally find neologisms; for these a separate heading is provided. Possibly their occurrence may be taken as an indication of an exaggerated tendency to respond by sound reactions.

Word Complements.-Here we include any reaction which, added to the stimulus word, forms a word, a proper name, or a compound word in common use.

Particles of Speech.-Under this heading we include articles, numerals, pronouns, auxiliary verbs, adverbs of time, place and degree, conjunctions, prepositions, and interjections.

Complete Dissociation.-Here are included reactions which appear to be entirely unrelated to the corresponding stimulus words; in the case of such reactions the stimulus words seem to act, as Aschaffenburg has pointed out, merely as signals for discharge. This subdivision contains several types of reactions which seem to be dependent upon the phenomenon of perseveration; it contains also the rather important type of neologisms. 
The phenomenon of perseveration occurs in cases in which one may observe an abnormal immobility of attention. To react normally to a series of stimulus words requires on the part of the subject, in the first place, a certain alertness in order that he may grasp quickly and clearly the meaning of each word, and, in the second place, a prompt shifting of the mind from one reaction to the next. When such mental mobility is lacking the subject is liable to react not by a response adjusted to the stimulus word, but either by repeating a previous stimulus or reaction, or by giving a word associated to the preceding stimulus or reaction.

The names of the different types of reactions included under the heading of perseveration are sufficiently descriptive; we shall here refer only to those which require further definition.

Association to Preceding Stimulus.-Here is placed any reaction that is shown by the frequency tables to be related to the stimulus preceding the one in question. Seeming or even obvious relationship, if not established by reference to the frequency tables, is desregarded. In the tables, however, the combination may not exist in direct order but only in reverse order, in which case the reaction is included here. The following examples may serve as illustrations:

$$
\begin{aligned}
& \text { thief-night } \\
& \text { lion-pocket-book }
\end{aligned}
$$

Lion-pocket-book is not found in the frequency tables, and is, therefore, an individual reaction; thief-pocket-book, however, is found there; pocket-book is, therefore, classed in this case as an association to preceding stimulus.

$$
\begin{aligned}
& \text { table-fork } \\
& \text { dark-mutton }
\end{aligned}
$$

Dark-mutton is not found in the frequency tables; table-mutton is also not found there in the direct order, but is found in the reverse order, viz.: mutton-table; mutton is, therefore, classed in this case as an association to preceding stimulus.

Association to Preceding Reaction.-If either the reaction in question or the preceding reaction happens to be one of the stimulus words in our list, and a relationship between the two be found to exist by reference to the frequency tables-whether in direct or in reverse order-the reaction in question is classed as 
an association to preceding reaction. This is illustrated by the following examples:

$$
\begin{aligned}
& \text { eating-table } \\
& \text { mountain-floor }
\end{aligned}
$$

Mountain-floor is an individual reaction; table-floor is found in the frequency tables; floor is, therefore, classed as an association to preceding reaction.

$$
\begin{aligned}
& \text { beautiful-flowers } \\
& \text { window-red }
\end{aligned}
$$

Window-red is an individual reaction; red-flowers is found in the frequency tables; therefore, red is classed as an association to preceding reaction.

In cases in which neither the reaction in question nor the preceding reaction happens to be one of our stimulus words, but a relationship between them may be judged to exist without considerable doubt, the reaction in question is also classed here. Example:

$$
\begin{aligned}
& \text { pricst-father } \\
& \text { ocean-mother }
\end{aligned}
$$

Ocean-mother is an individual reaction; neither the word father nor the word mother is among our stimulus words; but the association between the words father and mother may be judged to exist without considerable doubt; therefore, in this case mother is classed as an association to preceding reaction.

In such cases as this personal equation must necessarily come into play; comparative uniformity of judgment may, however, be attained by systematically excluding any reaction the relationship of which to the preceding reaction is subject to any considerable doubt and by placing any such reaction in the unclassified group.

Repetition of Previous Stimulus.-Here we place any reaction which is a repetition of any previous stimulus from amongst the ten next preceding, at the same time placing repetition of preceding stimulus under a separate heading.

Neologisms.-Here we place the newly coined words, so commonly given by the insane, excepting such as possess a sound relationship to the stimulus word, for which, as already stated, a special place in the classification has been provided. 
Neologisms might be divided into three types, as follows: (I) those which arise from ignorance of language (comfort-uncomfort, short-diminiature); (2) distortions of actual words, apparently of pathological origin and not due to ignorance (hungry-foodation, thief-dissteal); and (3) those which seem to be without any meaning whatever (scack, gehimper, hanrow, dicut). It is, however, impossible to draw clear-cut distinctions between these types, and for this reason we have made no provision in our classification for such division.

Unclassified Reactions.-This group is important, in the first place, because it is numerically a large one, and in the second place, because it contains certain fairly definite types of reactions which are placed here for the sole reason that we have not been able to find strictly objective criteria for their differentiation from other types.

It has already been stated that the frequency tables, even together with the appendix, fail to exhaust all normal possibilities of association, so that a certain small number of perfectly normal reactions must fall into the unclassified group. We submit the following examples:

$$
\begin{aligned}
& \text { music_listen } \\
& \text { smooth-suave } \\
& \text { sour-curdled } \\
& \text { earth-mound }
\end{aligned}
$$

Another type of reactions found in the unclassified group, though also normal, yet not obviously so until explained by the subject, is represented by those which originate from purely personal experiences, such as the following, given by normal subjects:

$$
\text { blossom-T . . . . }
$$

The first of these reactions is explained by the subject's acquaintance with a young lady, Miss T . . . ., who has been nicknamed "Blossom," and the second is explained by the subject's having among her pupils at school a boy by the name of $\mathrm{J}$... . Hammer.

It would be difficult to estimate the proportion of such reactions in the unclassified group, but we have gained the general 
impression that it is small. An attempt to place them in a separate group could be made only with the aid of explanations from the subjects; such aid in the case of insane subjects is generally unreliable. Moreover, to class these reactions as strictly normal would perhaps be going too far, since their general value is obviously inferior to that of the common reactions; and in any case in which they are given in unusually large numbers they must be regarded as manifestation of a tendency to depart from the normal to the extent to which they displace common reactions.

The next type of reactions met with in the unclassified group is characterized by a peculiarly superficial, or non-essential, or purely circumstantial relationship to the stimulus. Such reactions, though occasionally given by normal subjects, are more often given by insane ones, and seem to be somewhat characteristic of states of mental deterioration which are clinically rather loosely described as puerilism. We offer the following examples, given by normal subjects:

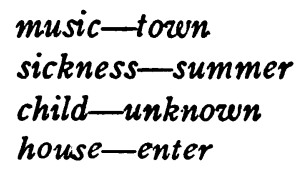

Still another type of reactions to be considered in this connection consists of words which are in no way related to the corresponding stimulus words, but which arise from distraction of the subject by surrounding objects, sounds, and the like. In some cases the experimenter may be able to judge from the direction of the subject's gaze, from a listening attitude, and so on, that certain reactions are due to distraction. In other cases, particularly in cases of normal subjects, the fact that certain reactions are due to distraction may be determined by questioning the subject on this point immediately after making the test. In work with insane subjects, as we have several times had occasion to point out, such aid is generally not available.

The group of unclassified reactions includes also one more type of reactions which are of great importance both numerically and otherwise. These are the incoherent reactions, that is to say, reactions which are determined neither by the stimulus words, nor by the agency of perseveration, nor by distraction. 
Although the occurrence of incoherent reactions is hardly subject to doubt, yet in no instance is it possible to establish with certainty that a given reaction is of this type, for in no instance can a remote, or an imagined, or a merely symbolic relationship between stimulus and reaction be positively excluded. Some, indeed, would assert that some such relationship must necessarily exist in every instance, at least in the domain of the subconscious. This circumstance necessitates the placing of this type of reactions in the unclassified group.

In practice it may be found advisable in some cases to analyze the unclassified reactions with a view to ascertaining to what exent each of the various types is represented among them. But one here treads on slippery ground, and one must be continually warned against the danger of erroneous conclusions.

\section{§ 5. Order of Preference.}

After having developed the classification here proposed we found that there was still considerable room for difference of opinion in the placing of many reactions, owing to the circumstance that in many cases a reaction presents features which render it assignable under any one of two or more headings. To leave the matter of preference in grouping to be decided in each case according to the best judgment of the experimenter would mean introducing again the play of personal equation, and would thus court failure of all our efforts to accomplish a standardization of the association test. Therefore, the necessity of establishing a proper order of preference for guidance in the application of the claissification became to us quite apparent.

In the arrangement of the order of preference we were guided mainly by two principles, namely: (I) as between two groups of unequal definition, the one which is more clearly defined and which, therefore, leaves less play for personal equation is to be preferred; (2) as between two groups of equal definition, the one which possesses the greater pathological significance is to be

preferred. In accordance with these principles we have adopted the order of preference shown in Table III., placing every reaction under the highest heading on the list under which it may be properly classed. 
TABLE III.

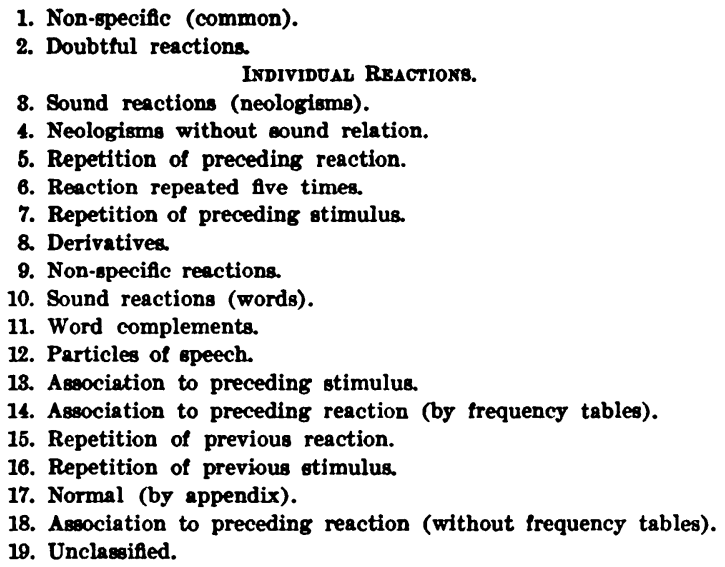

\section{§6. Errors Involved in the Use of Arbitrary Objective STANDARDS.}

It may readily be seen that such definiteness and uniformity as this classification possesses results from the introduction of more or less arbitrary criteria for the differentiation of the various types of reactions. The question might arise, To what extent do the distinctions thus made correspond to reality? To consider, for instance, our rule for the placing of sound reactions (50 per cent of the sounds of the shorter word to be present, in the same order, in the other word) : when a given reaction (man-minstrel) is in accordance with the rule assigned under the heading of sound reactions, can it be assumed that sound similarity and not some other relationship is the determining factor of the association in question? Or when in a given instance (cabbage-cobweb) the sound similarity falls somewhat short of the standard required by the rule, can it be assumed that sound similarity is not, after all, the determining factor?

Similar questions may, of course, arise in connection with other subdivisions.

It must, indeed, be conceded that objective methods can reveal but indirectly and with uncertainty the inner mechanism which produces any association and that in any given instance it would be impossible to establish the correctness of grouping in accordance with such methods. However, to decide that question for any given reaction is really not necessary in practice, since an error 
made through wrongly placing one, two, or three reactions under any heading is of no significance; the types acquire importance only when represented by large numbers in a record under consideration; and when many reactions fall under a single heading the likelihood of error, as affecting the record as a whole, is by that fact alone greatly reduced.

The whole question might more profitably be approached from another point of view: To what extent are the distinctions of this classification useful? An answer to this question can be found only in the results.

\section{§ 7. Analysis of Pathological Material.}

We present in Table IV. the results of a statistical examination of the records obtained from certain groups of normal subjects and from some groups of insane subjects.

The normal groups have been studied for the purpose of determining the frequency and manner of occurrence among normal subjects of the various types of abnormal reactions. It seemed best for this purpose to consider separately the records of those subjects who gave an unusually large number of individual reactions. Fifty-three records containing fifteen or more individual reactions were found after a fairly diligent search among our normal test records. In the other groups of subjects-persons of common school education, persons of collegiate education, and children-we included no records containing more than ten individual reactions.

The more striking departures from average normal figures are indicated in the table by the use of heavy type.

This table reveals some special associational tendencies as occurring in connection with the psychoses studied. A better insight into the nature of these tendencies can be gained by a special analysis of the test records of each clinical group.

\section{DEMENTIA PRACOX.}

In this psychosis we find the average number of individual reactions far exceeding not only that of the normal but also that of any other psychosis which we have studied. To a corresponding extent we find the average number of the highest type of normal reactions-the common specific reactions-reduced.

While almost every type of individual reactions shows here an 


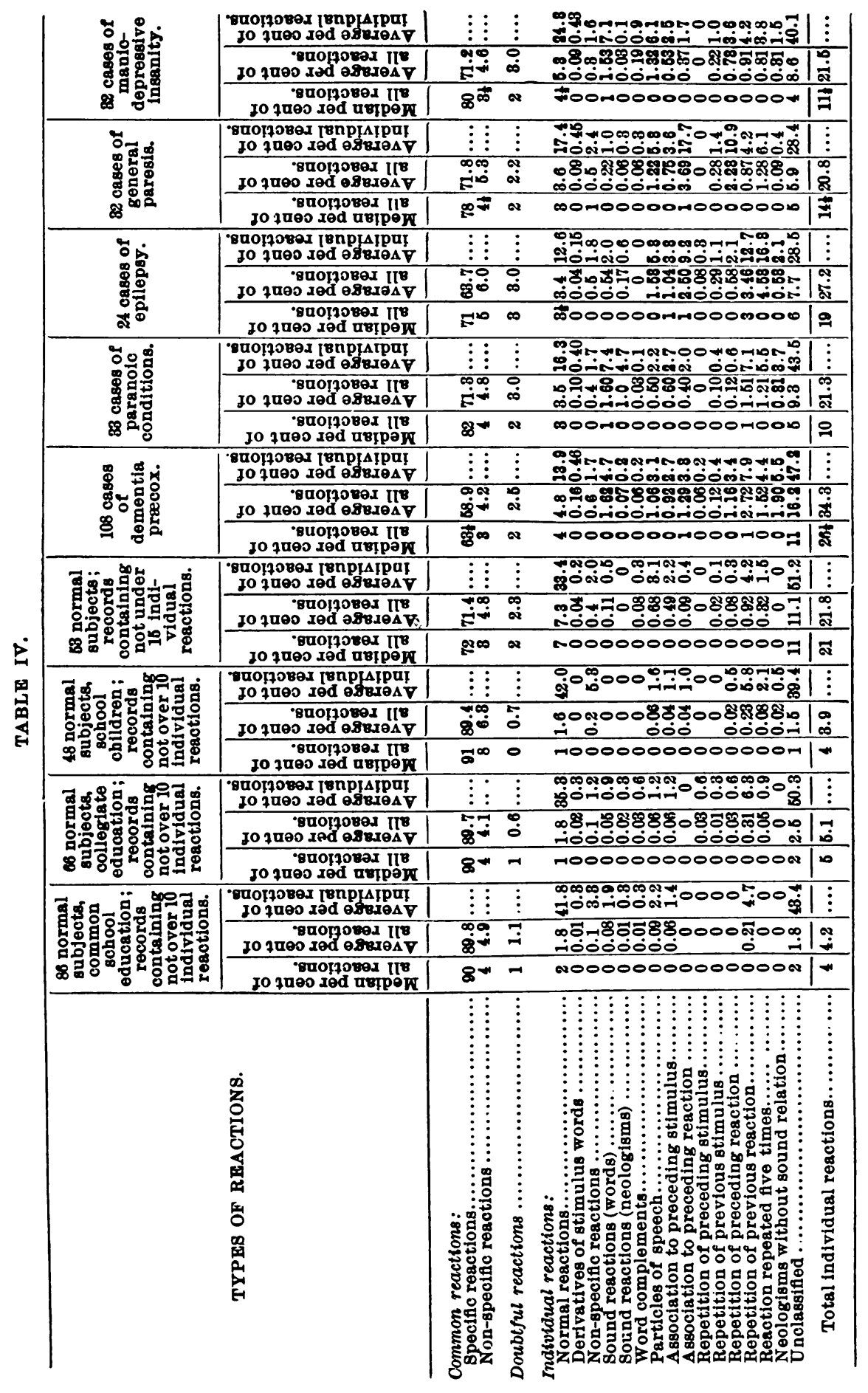


increase over the normal averages, the most striking increases are shown by the table to be in the groups of unclassified reactions, neologisms, sound reactions, and some types of perseveration. A further examination of the individual test records shows that there is no uniformity of associational tendencies in this clinical group, but that several tendencies are more or less frequently met with either alone or in various combinations. Yet some of these tendencies, when appeáring at all prominently, are so highly characteristic of dementia præcox as to be almost pathognomonic. Among these may be mentioned: ( $\mathrm{r}$ ) the tendency to give neologisms, particularly those of the senseless type; (2) the tendency to give unclassified reactions largely of the incoherent type; and (3) the tendency toward stereotypy manifested chiefly by abnormally frequent repetitions of the same reaction. Fairly characteristic also is the occasional tendency to give sound reactions. Again, occasionally one encounters pronounced perseveration, and at least two of our subjects gave a good many unclassified reactions obviously due to distraction.

It must be noted that not infrequently cases of dementia præcox give test records that cannot be distinguished from normal. It seems that the pathological associational tendencies constitute merely a special group of symptoms, some of which may be expected to be manifest in cases which have reached a state of advanced mental deterioration, but may not necessarily be present in the early stages of the disease. On the other hand there is evidence to show that these tendencies may in some cases appear among the earliest manifestations. This matter will be referred to again.

Thus the test records of dementia præcox depart from the normal not sharply but by a gradual shading off. We find similar gradual transitions between dementia præcox and other psychoses. For this work we selected cases in which the diagnoses were established with reasonable certainty. Whether or not in cases of doubtful clinical classification this association test may be of aid in determining the diagnosis, is a question that must for the present remain open.

We submit herewith copies of test records. The numbers which appear after the reactions indicate in each case the reaction type, in accordance with Table III. (p. 328); common specific reactions are not numbered. 
CAse No. 4752.-H. J. Neologisms; some unclassified reactions, mostly incoherent.
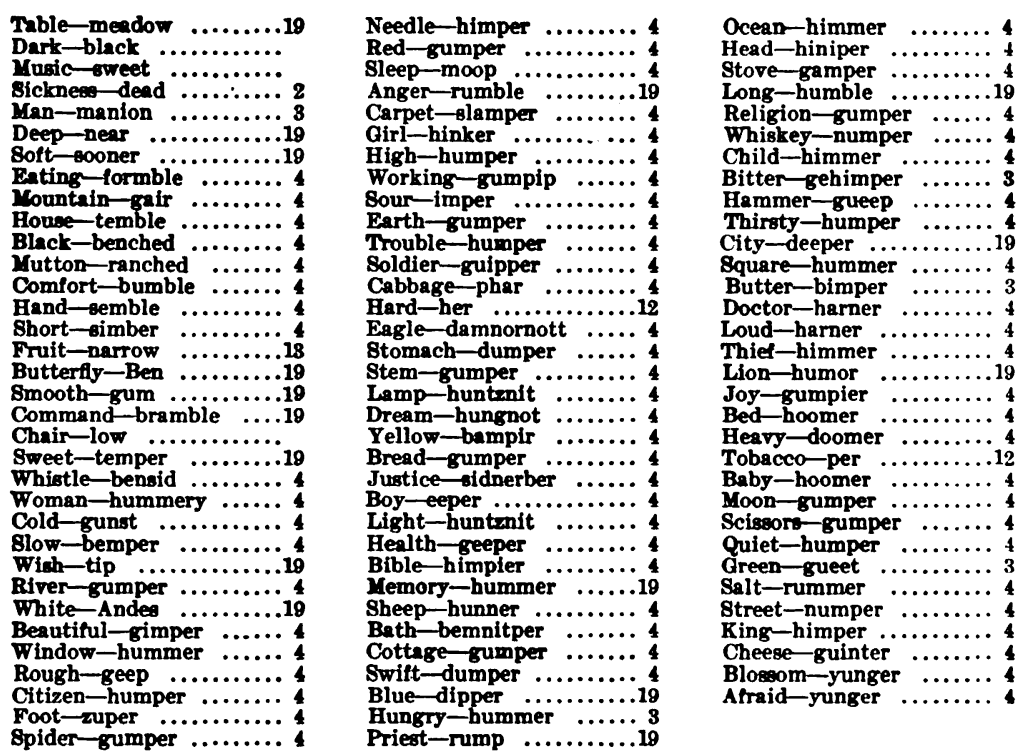

Case No. 5183-G. D. Neologisms; numerous unclassified reactions, mostly incoherent; some sound neologisms.
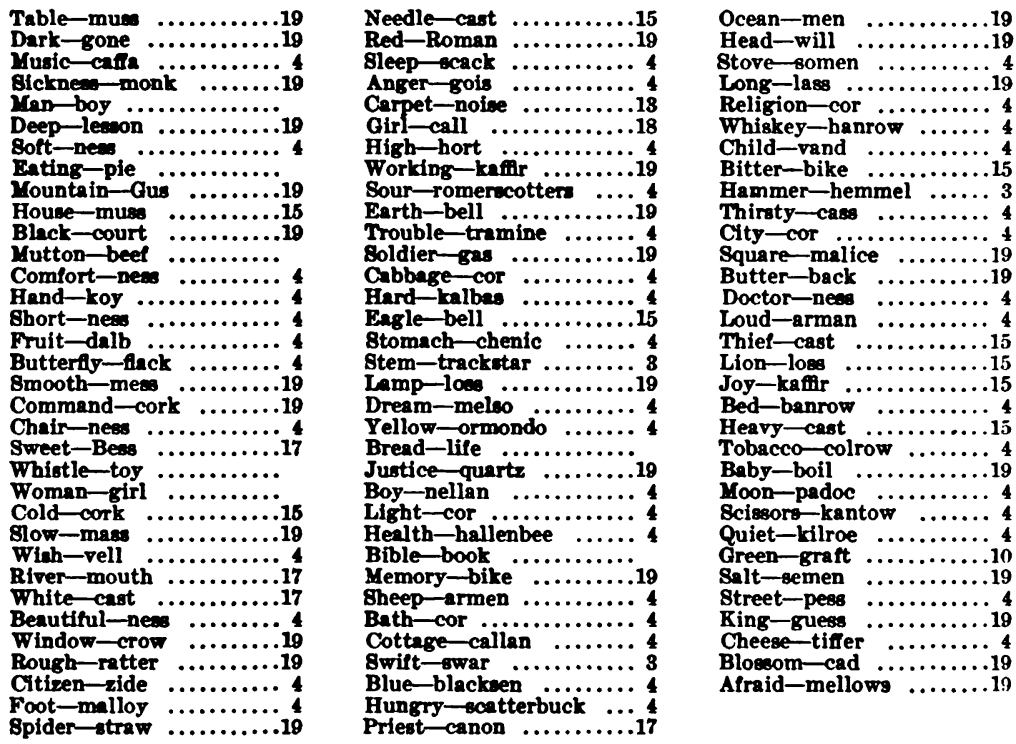
CASE No. I500.-D. V. Considerable number of neologisms; stereotypy manifested partly in a tendency toward frequent repetition of certain reactions but mainly in a persistent tendency to make use of the grammatical form of present participle, giving rise to numerous doubtful reactions.
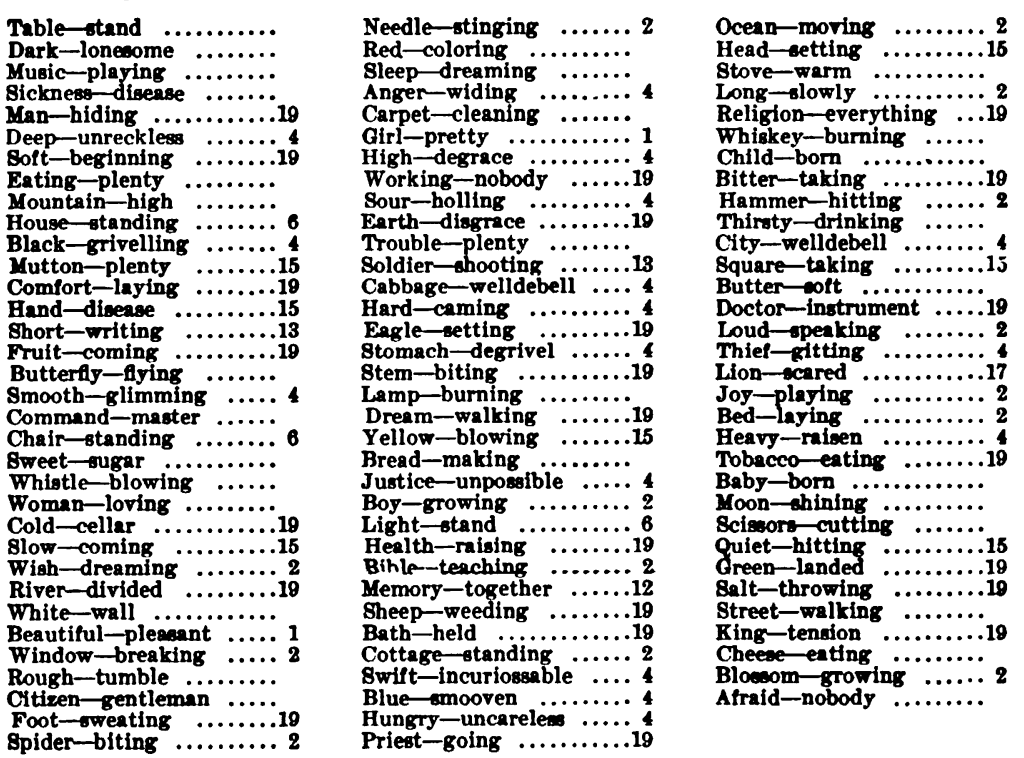

CASE No. 5138.-C. J. Unclassified reactions, mostly incoherent.
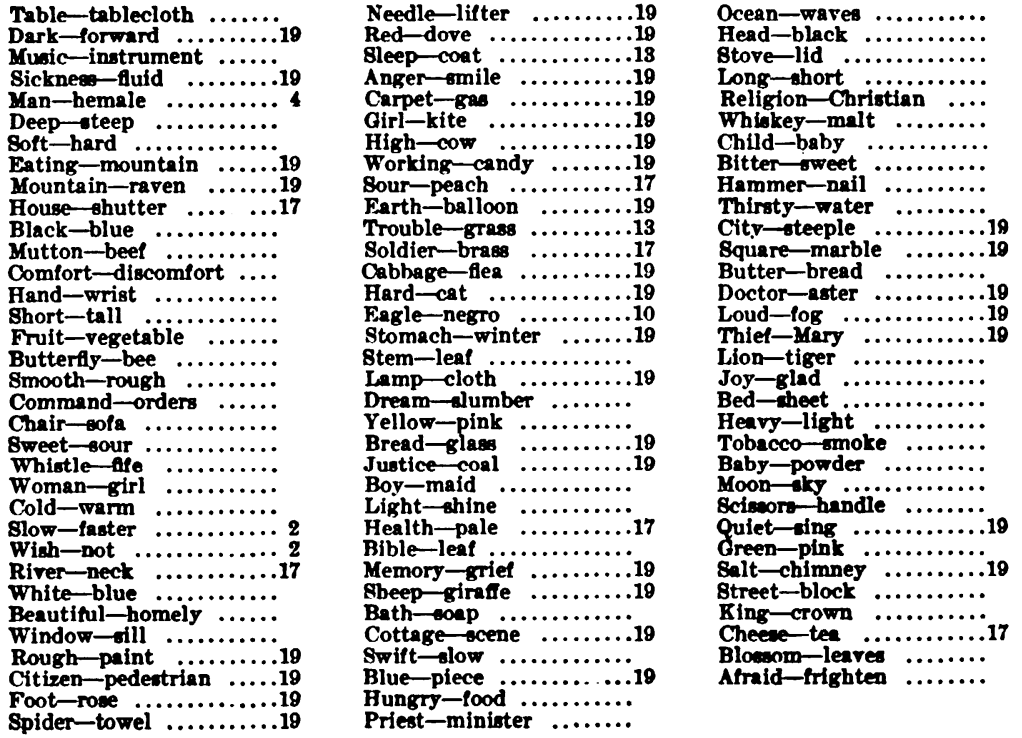

Priest-minister .......... 
CASE No. I7979.-R. T. Unclassified reactions, mostly incoherent.
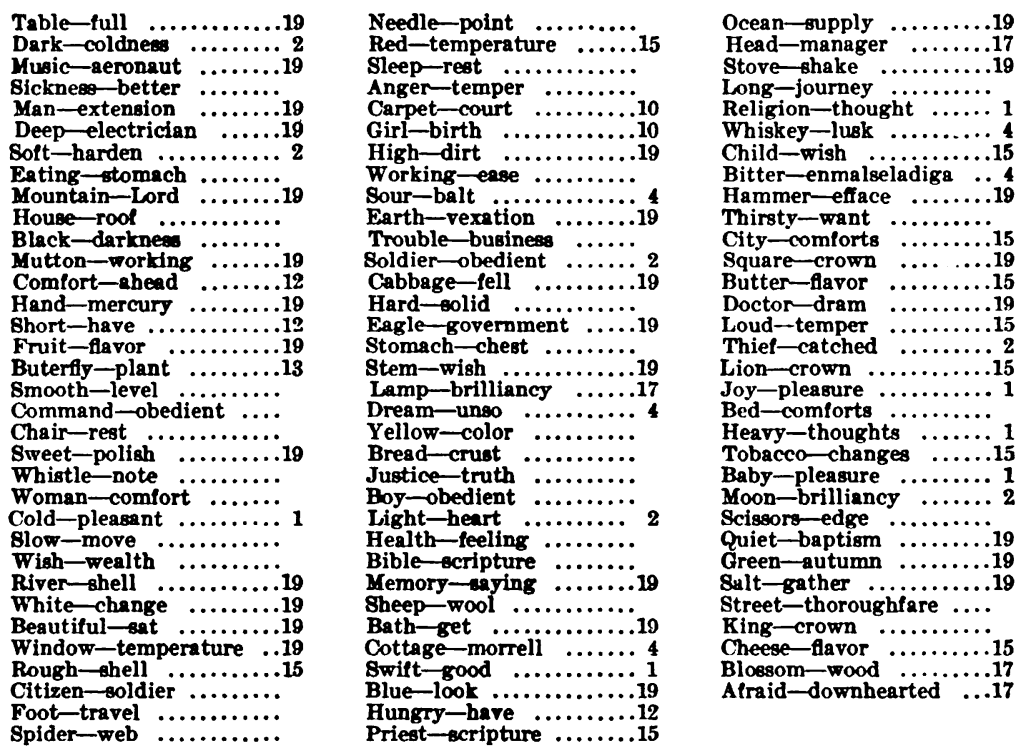

CASE No. 3307.-G. F. Unclassified reactions, mostly incoherent; slight tendency to respond by sound reactions.
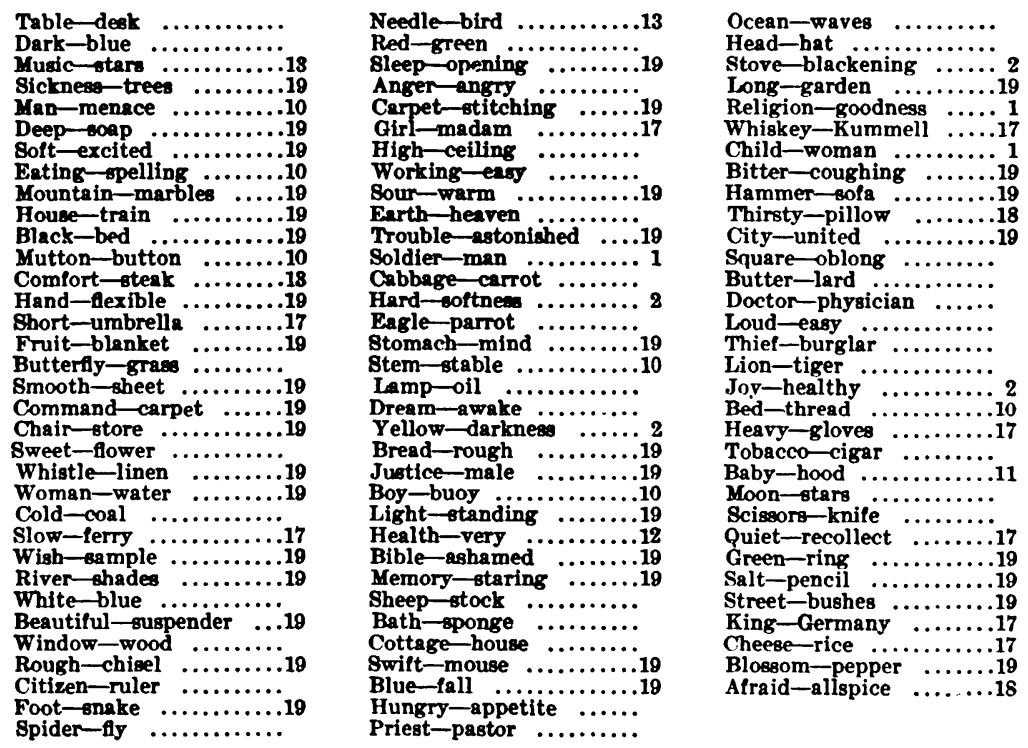
CASE No. 971.-O. M. Unclassified reactions, mostly incoherent.
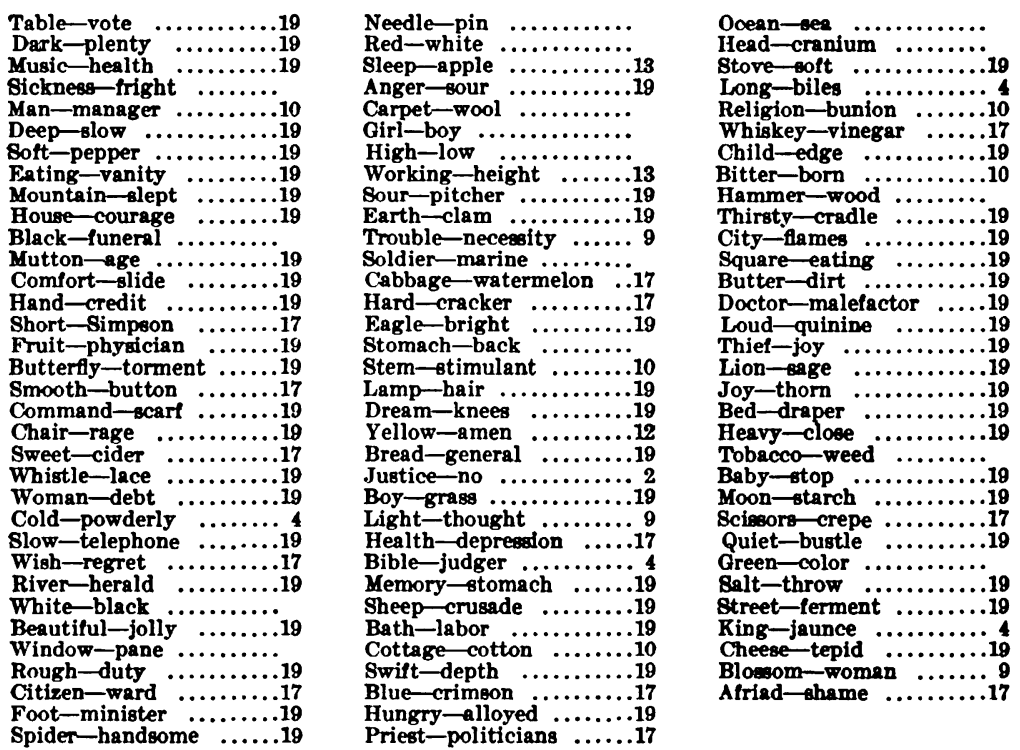

CASE No. ol655-E. H. Unclassified reactions, mostly incoherent.
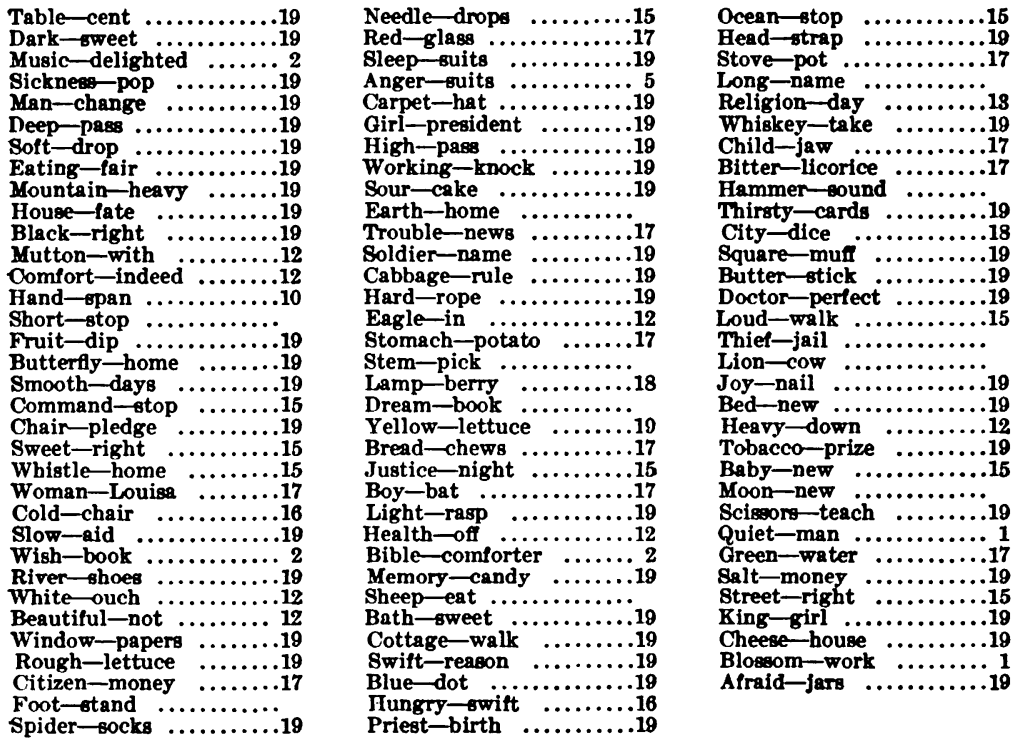
Case M. F. (from Hudson River State Hospital).-Unclassified reactions, mostly incoherent.
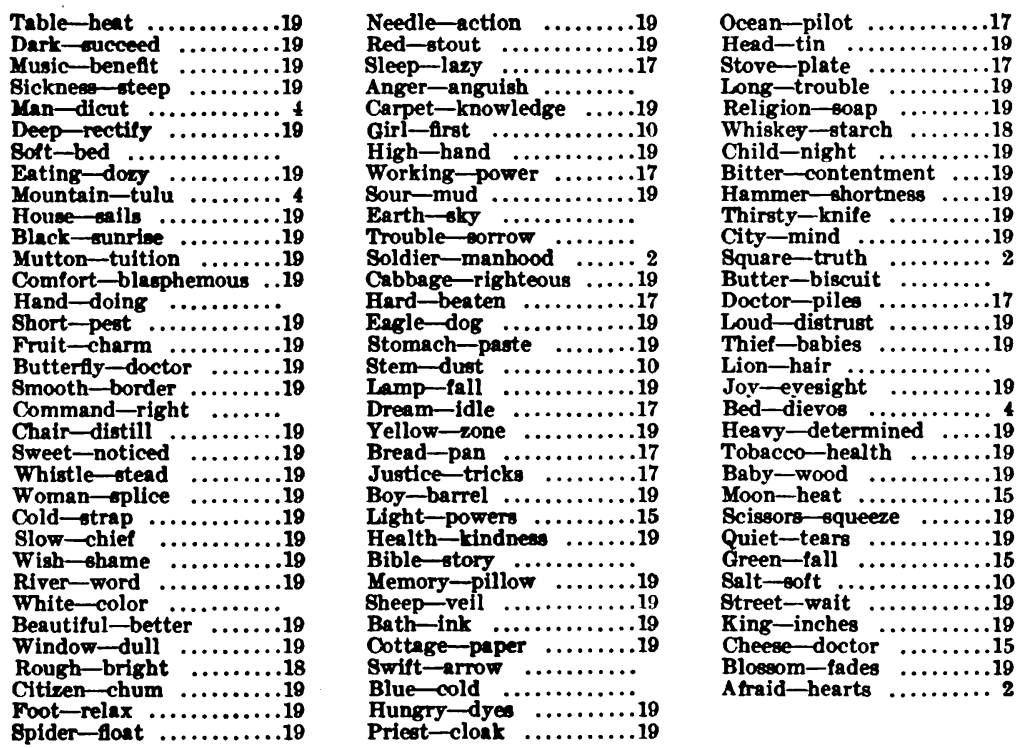

Case No or552.-E. J. D. Unclassified reactions, mostly incoherent.
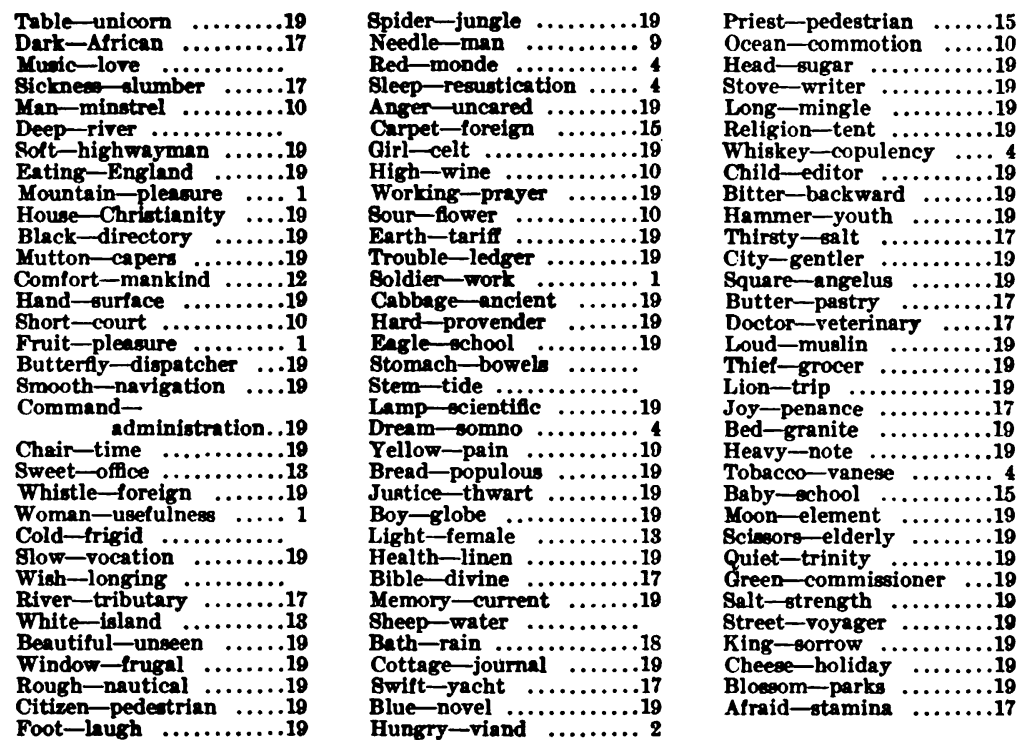
Case No. 667.-C. L. Pronounced stereotypy. Following note on test record: "Many attempts were made to secure a reaction other than ' cat,' but usually without success; the reaction cold-warm was given spontaneously and with apparent interest; most reactions were given only in response to much urging, or else mechanically, without attention."
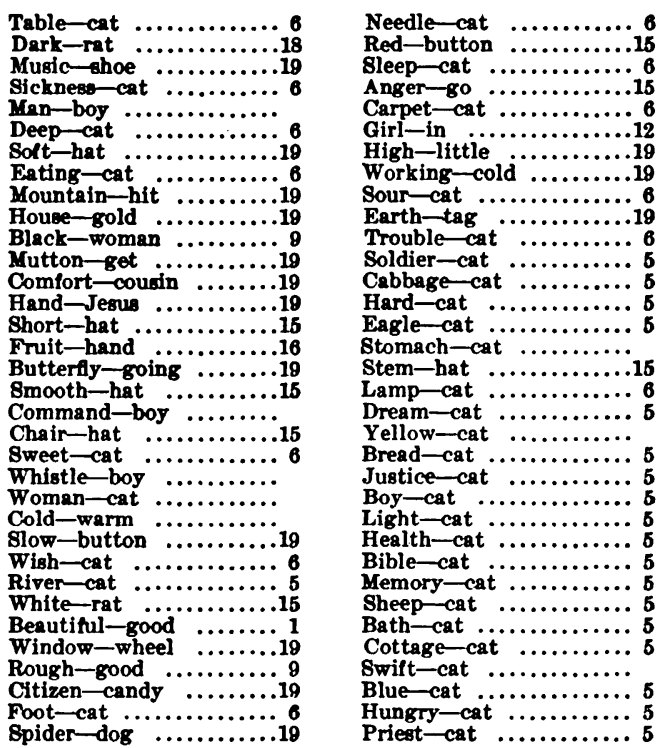

Case No. 6006.-E. T. S. Stereotypy.

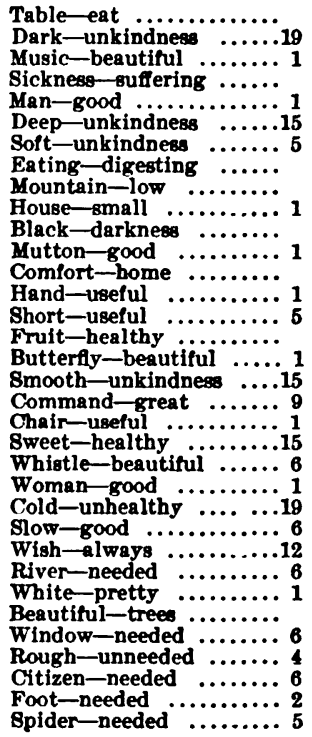

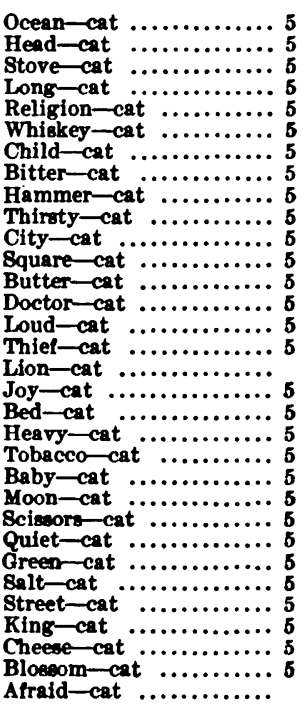

Ocean-Presh $\ldots \ldots \ldots \ldots 19$ Head-unhealthy $\ldots . \ldots .15$ Stove-warmth $\ldots \ldots \ldots \ldots$. Religion-needed $\ldots . . . . .$. Whiskey-needed $\ldots \ldots . . .25$ Child-needed Bitter-needed ..........5 Hammer-needed .......5 5 Thirsty-water City-pretty .............. Squere-honest Butter - good....$\cdots \cdots \cdots \cdots$ Doctor-needed ............. Loud-needed ........... 5 Thief-trust Lion-love . . ...............19 Joy-laughter Bed-comfortable .......... Heavy $\rightarrow$ leepiness $\cdots \cdots \cdots$. 2 Tobacco-needed ........ Baby-needed...$\ldots \ldots \ldots$. Moon-needed $. \cdots \ldots \ldots . .5$ Scissor - needed ........ Quiet-pleasure ......... 1 Green-me $\ldots \ldots \ldots \ldots \ldots$ Salt-needed ............ Street-needed...$\ldots \ldots \ldots$. Cheese-needed ........... Blossom-needed .......... Afraid-nervous ...........

\begin{tabular}{l}
5 \\
5 \\
5 \\
5 \\
5 \\
5 \\
5 \\
5 \\
5 \\
5 \\
5 \\
5 \\
5 \\
5 \\
5 \\
5 \\
\hline 5 \\
5 \\
5 \\
5 \\
5 \\
5 \\
5 \\
5 \\
5 \\
5 \\
5 \\
5 \\
5 \\
5 \\
\hline
\end{tabular}


CASE No. 2292.-C. M. Perseveration: numerous instances of association to preceding reaction; unclassified reactions, mostly incoherent.
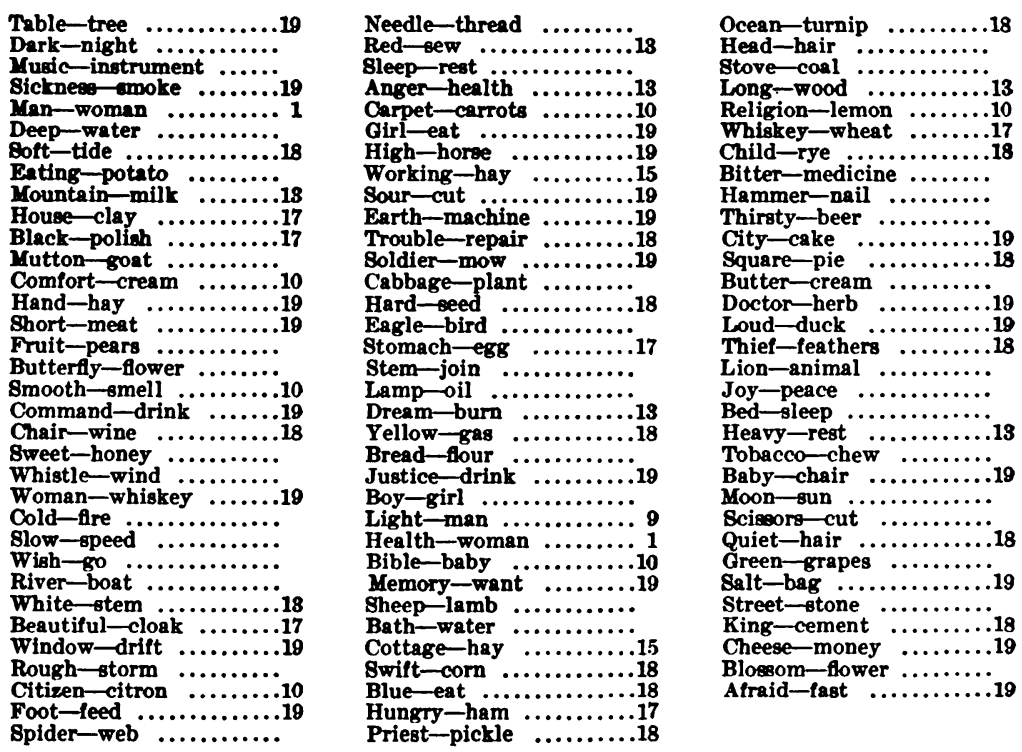

CASE No. 17880.-E. D. Numerous repetitions of reactions previously given; unclassified reactions, mostly incoherent; neologisms.
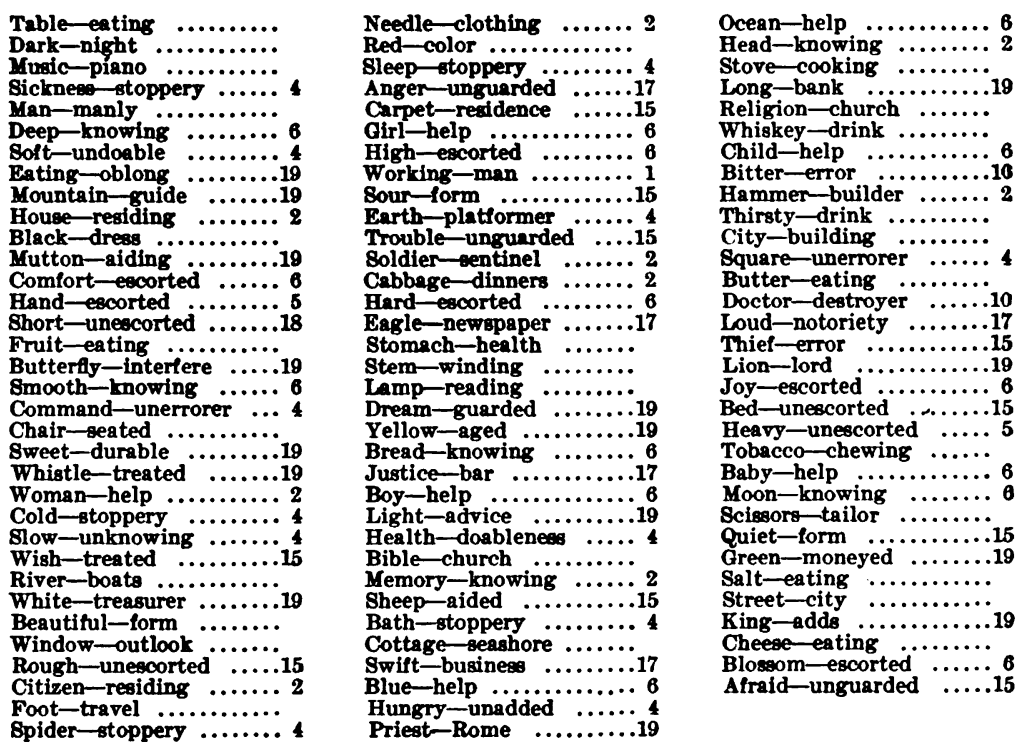

stoppery .............

Priest-Bome 
Case No. 6065-A. F. Unclassified reactions, mostly incoherent; perseveration: instances of association to preceding reaction and to preceding stimulus.
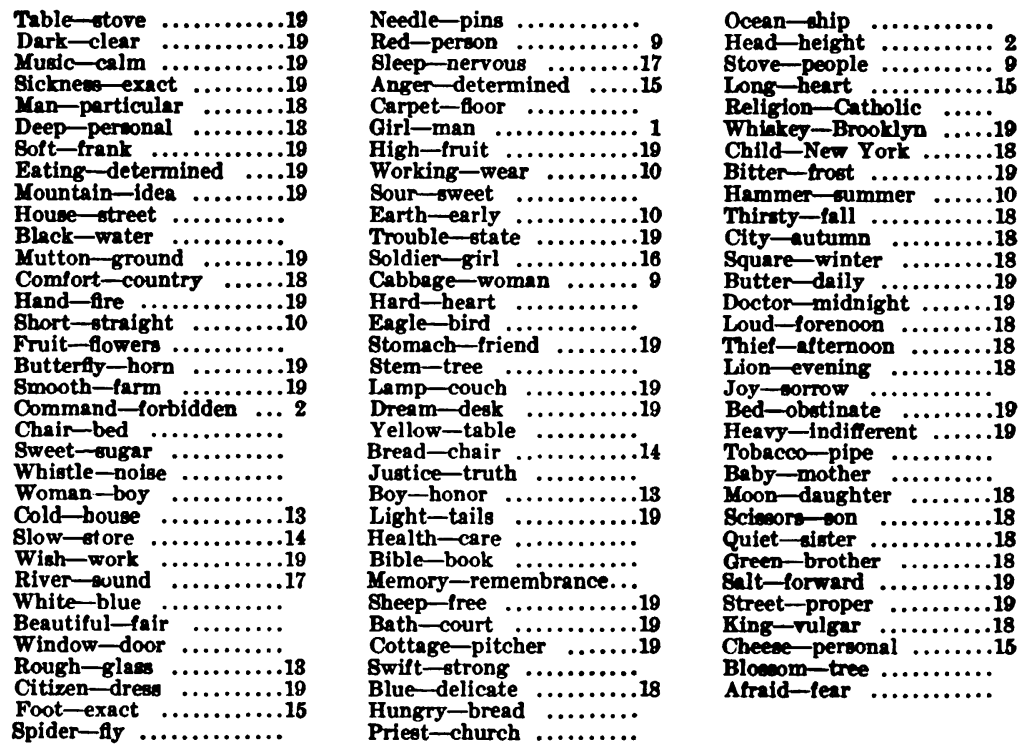

CASE No. 17188.-G. B. Sound reactions; unclassified reactions, mostly incoherent.
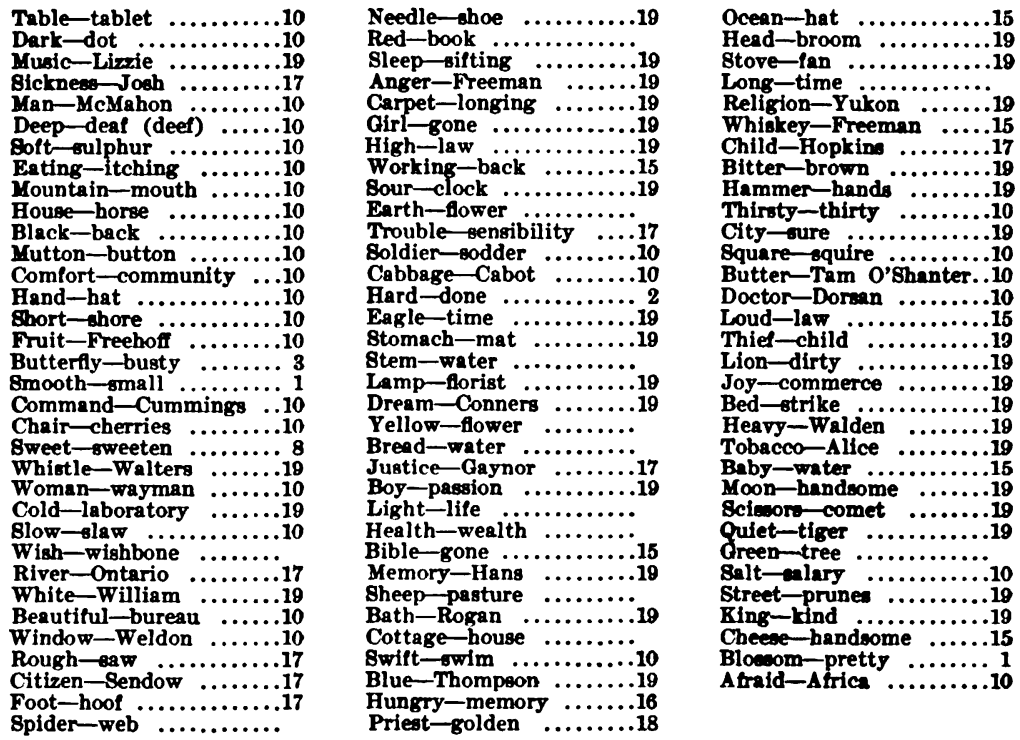
CaSe No. 6238.-M. H. Sound reactions; unclassified reactions, mostly incoherent.
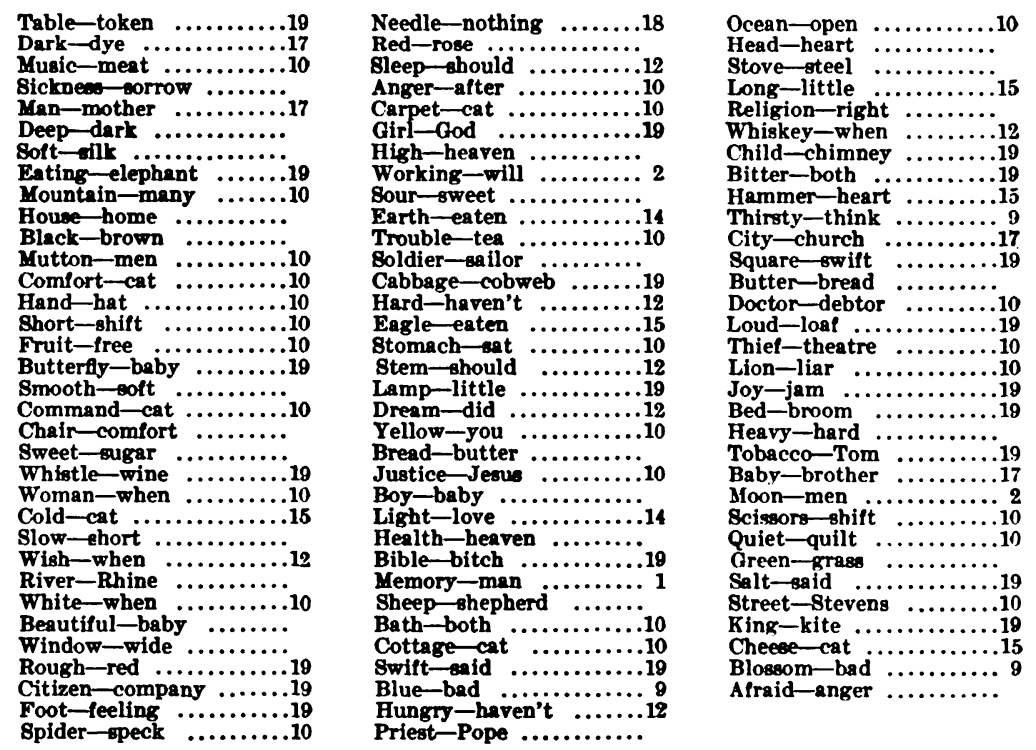

CASE No. 12720.-J. B. Unclassified reactions, many of which are probably due to distraction; some stereotypy. Note on test record states: "Influenced by sensory impressions, but gave good attention to each stimulus word. Had some difficulty in limiting his response to one word, but made all possible effort to comply with every request. On one occasion he was asked to react with his eyes closed, but was unable, under the unnatural conditions, to respond with one word."
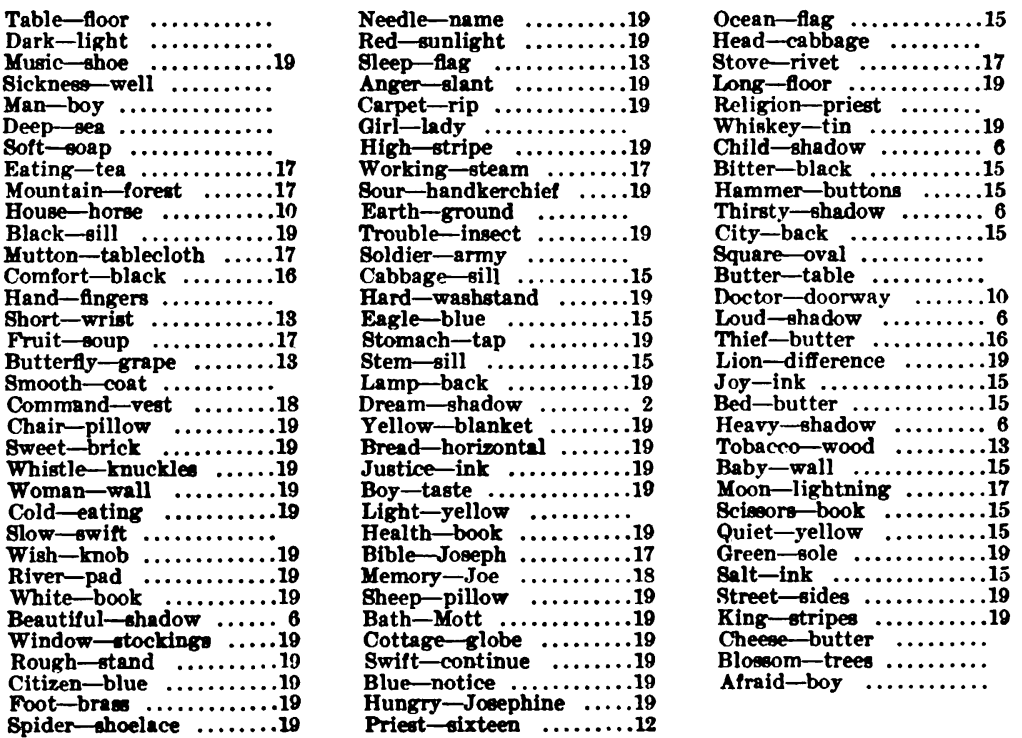
CAse No. 5374-J. F. Perseveration; some stereotypy; sound reactions; unclassified reactions many of which are probably due to distraction. Note on test record states: "Understood what was expected, but could not be induced to give much attention to the stimulus words; sat facing a window, and showed a strong tendency to merely name objects in sight. Reaction time very short, in some cases so short that it is doubtful if he recognized the stimulus word at all."
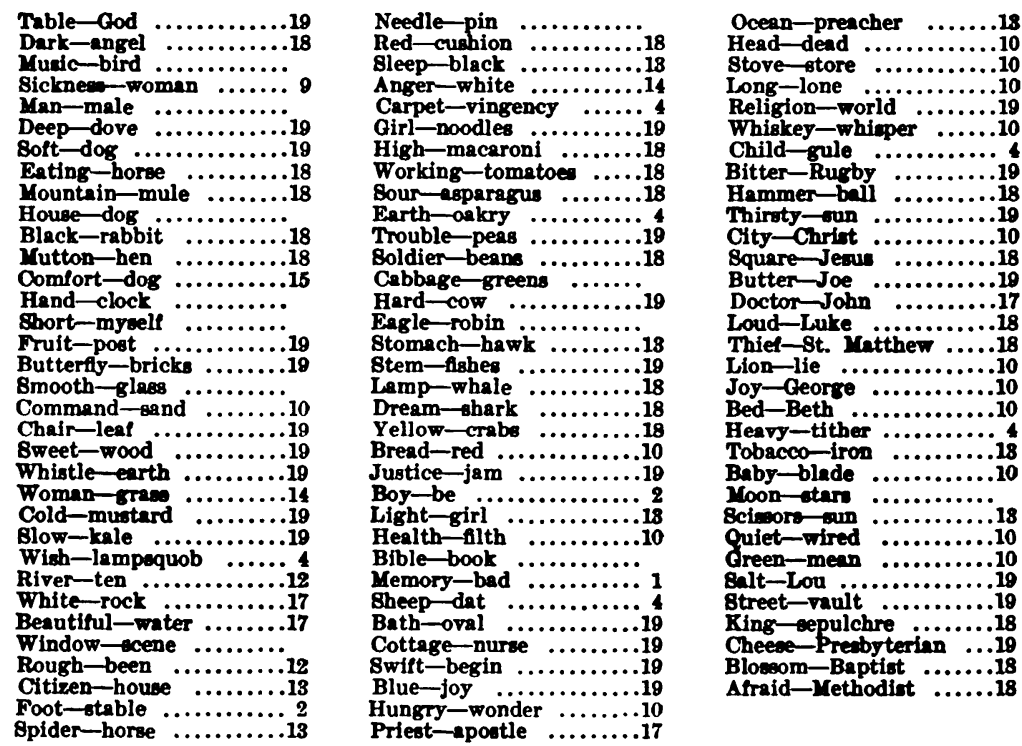

Case No. I431.-A. L. Sound reactions; particles; unclassified reactions, mostly incoherent.
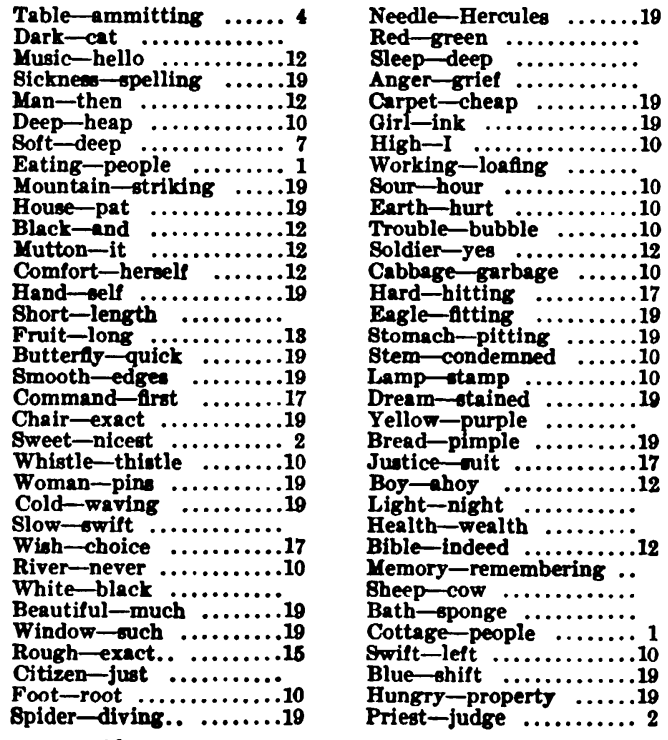

Ocean-river

Head-ises

Stove-Venus ............18

Long - hog $\ldots \ldots \ldots \ldots \ldots$. .........

Whiskey-gin $\ldots \ldots \ldots \ldots$.

Bitter-better $\cdots \ldots \ldots \ldots .10$

Hammer-happy

Thirsty-whiskey $\ldots \ldots \ldots .17$

Bquare-round

Butter -hut $\cdots \cdots \cdots \cdots$

Doctor-exercise ........19

Loud $\rightarrow$ ccounts ...........10

Thief-endlew ..........19

Lion-tiger .

Joy-Inst ................

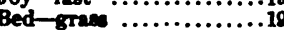

Heavy-heaving ..........10

Tobacco-queen $\ldots \ldots \ldots \ldots$.

Baby-water ............18

Moon-room ............19

Scismor-pants .........17

guiet-rasor ............18

Green-ateel ............18

Balt-sharp

Street-Pence .............19

King - bring .............10

Cheeso-eggs ...........

$\underset{\text { Afraid } \longrightarrow \text { waiting }}{\text { Bloss......19 }}$ 
CASE No. 625I.-C. D. Some stereotypy; particles; unclassified reactions, mostly incoherent.
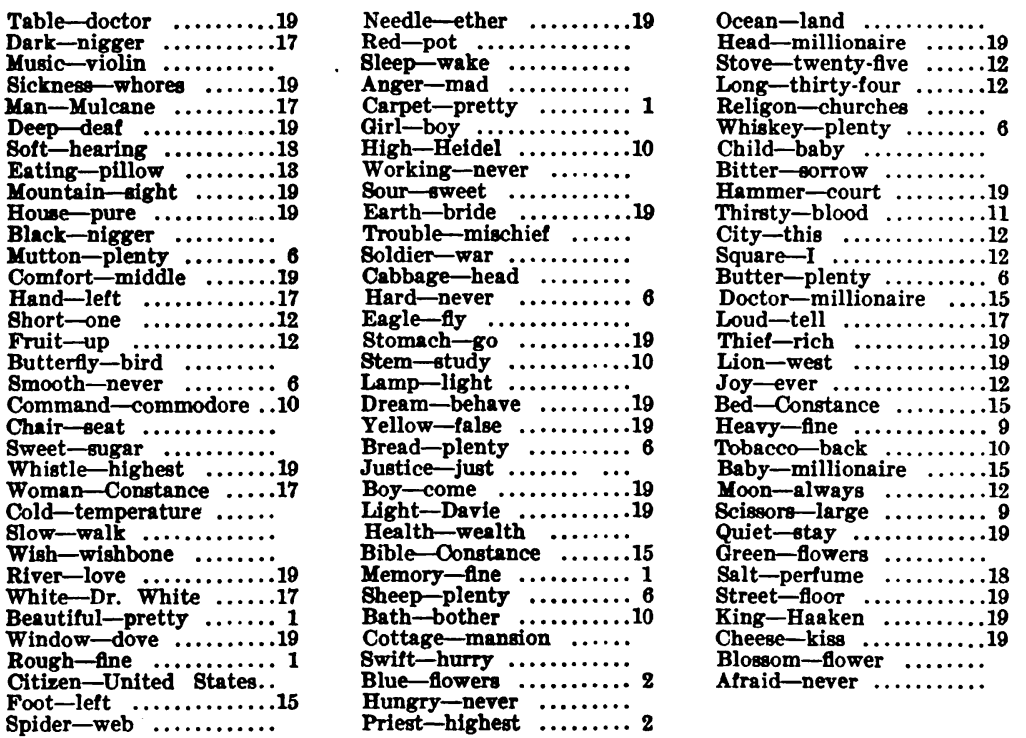

CASE No. 17607.-P. D. Test record somewhat approaching the normal: 24 individual reactions, of which 16 are unclassified, mostly "far fetched" and not strictly incoherent. Patient is a well-marked case of dementia præcox but only moderately deteriorated; works well at the hospital.

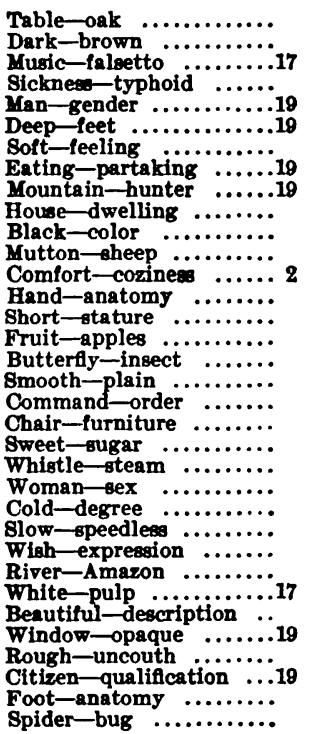

Needle - tteel

Red-color $1 . . . \cdots \cdots \cdots$

Aleep-rlumber $\ldots \ldots \ldots \ldots$.

Anger-aroused $\ldots . . . \cdots$.

Carpet-texture $\ldots \ldots \ldots$

Girl-female $\ldots \ldots \ldots \ldots$.

Wigh-up Working-doing $\ldots \ldots \ldots \ldots$

Working-doing $\quad . . \ldots \ldots .$.

Earth-dirt

Trouble-distres .........

Soldier-uniform .........

Cabbage - crop ..........

Hard-metal

Eagle-bird . ...............

Stomach-anatomy .....

Stem-pipe $\ldots \ldots \ldots \ldots \ldots$.

Lamp-glass .............19

Yream-atmosphere ......

Yellow-color

Bread-flour ...........

Justice - equality .......

Boy-male

hight-sun $\ldots . . . . . . . .$.

.

Bble-nonsense ........19

Ghop-quadruped

Sheep-quadruped

Bath-water $\ldots . . \cdots \cdots \cdots$

Swift-apeed

Blue-need

Blue-navy $\ldots . . . . . . . . .$.

Hungry-appetite $\ldots \ldots \ldots$
Priest-unform $\ldots \ldots \ldots 15$
Ocean-Atlantic $\ldots \ldots \ldots \ldots$.

Stove-iron . ..............

Long-inches . .........1

Religion-creed .........

Whiskey-hope $\ldots . . \cdots \cdots$

Child-neuter ..........19

Bitter-horehound ......

Hammer-steel $\ldots . . \ldots \ldots$.

Thirsty-degree ..........

City-population..$\ldots \ldots$

Square- ides

Butter-cream ..........

Doctor-physician ......

Thief-characterization...19

Lion-menagerie ........

Joy-openness ............

Bed-lurniture.........

Heavy - weight.......

Baby-egg $\ldots . . . \ldots \ldots \ldots \ldots$. 19

Ycisern

Quiet-noiseles

Quiet-noiseless ...........

Green-Paris $\ldots \ldots \ldots \ldots \ldots 11$

Street-lane $\ldots \ldots \ldots \ldots \ldots$

King-usurper ….........19

Closem - ............

Afraid-ocared 
Case No. 5537.-J. H. Test record approaching the normal: 2I individual reactions, 8 classed as normal, I non-specific, 12 unclassified, mostly "far fetched" but not strictly incoherent. Well-marked dementia præcox, but of recent origin and but slight deterioration.
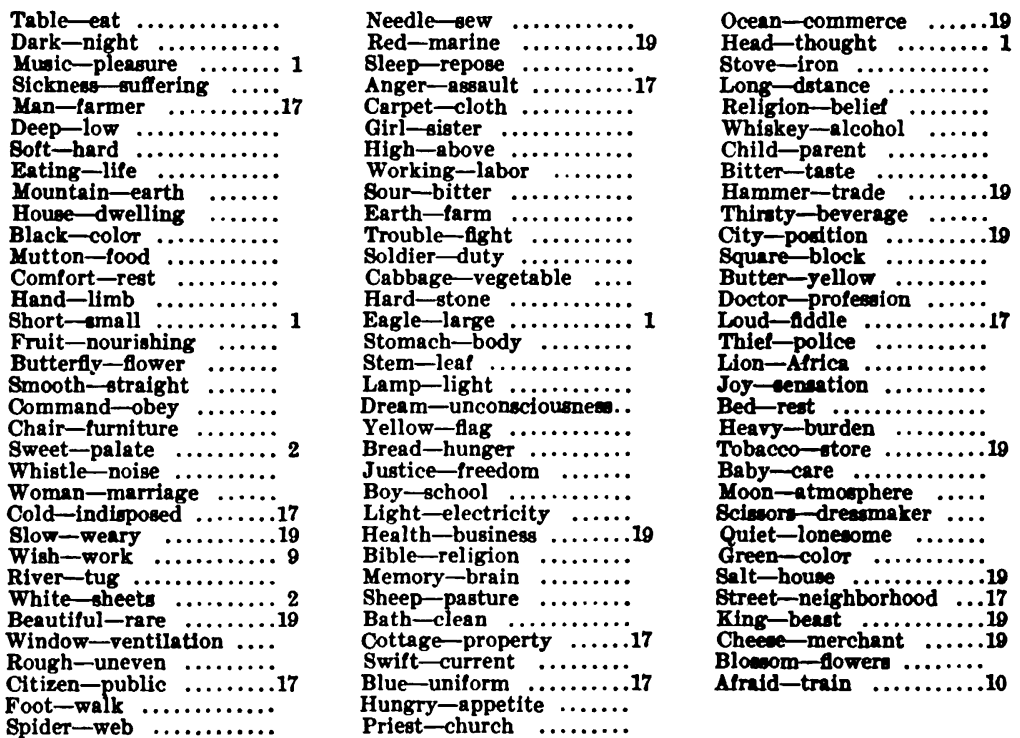

Case No. 6rgo.-L. L. Test record not distinguishable from normal. Case of recent onset, with little, if any deterioration.

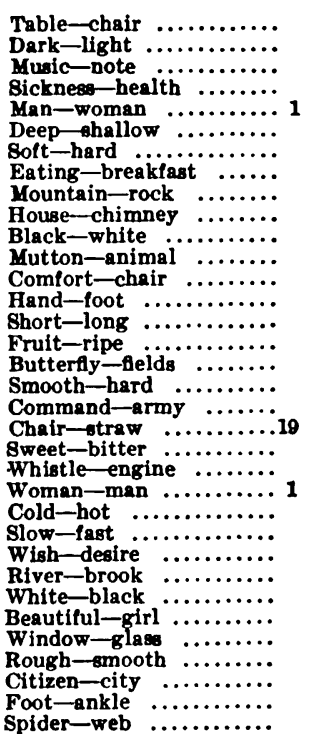

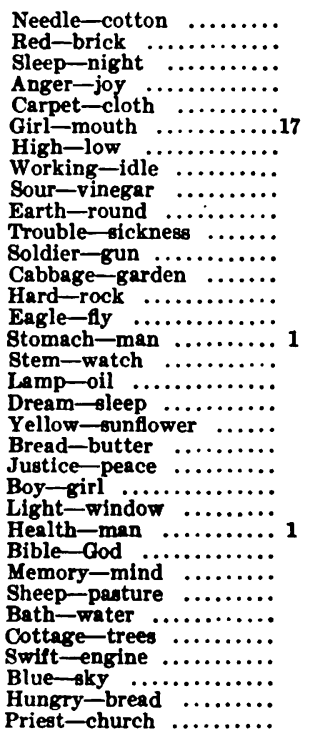

Ocean-shipe Stove-chimney $\ldots \ldots \ldots$......... Long-wind Religion-God . ............. Whiskey-alcohol ........ Child-mother ........... Bitter-fruit Hammer - nails $\ldots . . . . . .$. Thirsty-water City-cars .............. Qquare-angles $\ldots . . . .$. . Butter-cow Doctor $\rightarrow$ ckness....... . Loud-noise ... Thief-ginner..$\ldots \ldots \ldots$. 2 Lion-jungle oy-gladness ............. Bed-pillow Heavy-iron .............. Tobrcco-leap ............. Baby-mothe (a) ........... scisoro-thread ......... gulet-room

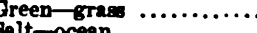

Greet-men $\cdots \cdots \cdots \cdots \cdots$

Sing - mueen...$\ldots \ldots \ldots$ Oneese-putter $\ldots \ldots \ldots$....... Blossom-bud Afraid-cowerd $\ldots . . . . .$. 
CASE No. 1278.-B. B. Test record not distinguishable from normal. Case of several years standing, but showing almost complete remission of all symptoms.
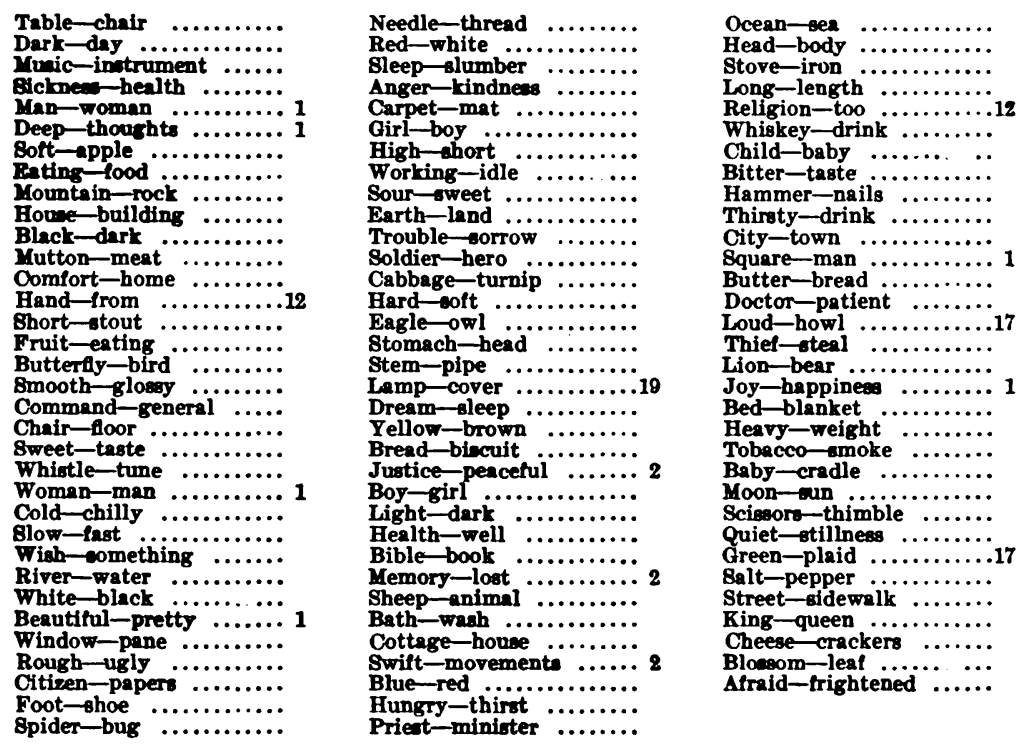

PARANOIC CONDITIONS.

The clinical group of psychoses included under the designation paranoic conditions is far from being homogeneous. We have here cases that are more or less closely allied to the paranoid form of dementia præcox, other cases that are apparently dependent upon involutional changes (Kraepelin's praeseniler Beeinträchtigungswahn), still other cases that are characterized by absence or at least delay of mental deterioration, etc.

In some of these cases disturbance of the flow of utterance is not observed, and the test records obtained from them present no striking abnormalities. Distinctly pathological records are obtained mainly from those cases which clinically resemble dementia præcox; in these records the nature of the pathological reactions would seem to indicate that the diagnosis of dementia præcox would be more justifiable than that of paranoic condition.

The following test records will serve to illustrate the types of reactions met with in this group of psychoses: 
Case No. 3039.-F. A. Normal record.
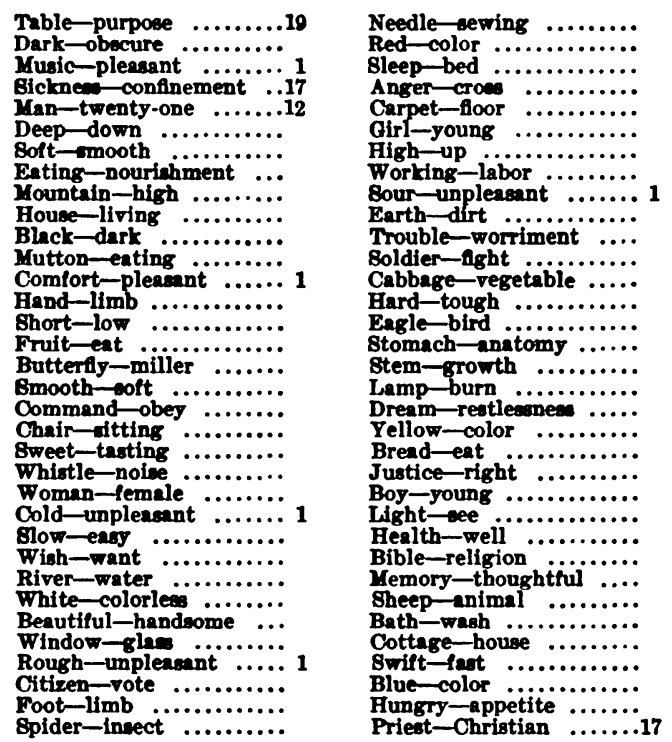

Ocenn-large $\ldots . . . \ldots . .1$

Head-trunk ............

Stove-fire ..............

Long-distance .........

Whinkey-drinkable ......

Ohild-roung.$\ldots \ldots \ldots \ldots \ldots$

Bitter -bad $\ldots \ldots \ldots \ldots \ldots$

Thirsty-dry ................

Olty - Government .......

gquare-block ............

Butter-eat

Doctor-cure $\ldots \ldots \ldots \ldots$.

Loud-noing

Thief-ateal

Lion-animal $\cdots . . . . .$.

Joy-pleasant $\ldots \ldots \ldots \ldots, \ldots$

Bed-laying $\ldots . . . \ldots \ldots . .22$

Heavy-weighty $\ldots . . . .$.

Tobacco-moking $\ldots \ldots \ldots$.

Baby-new-bor

Moon-planet ...........

Bcinorv-cutting .........

Qulet-easy

Green - color ............

Salt preservative .......

Btreet-Inne

King-monarch ..........

Blowom-budding .........

Atraid-fear .............

Priest-Christian $\ldots . . . .11$

CaSe No. 5803.-D. E. D. Slight tendency to give sound reactions.
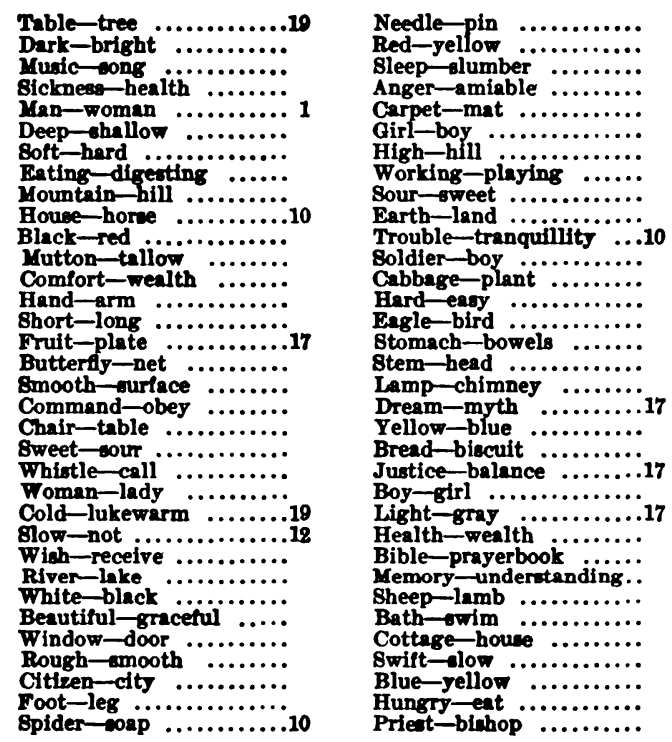

Oceen-river .............

Btove-neck . ..............

Long - short ..............

Religion-optional ........10

Whinkey-Fine .........

Child-baby

Bitter $\rightarrow$ weet ...........

Hammer-gimlet .......17

Thiruty-drink

City-town $. . . . \cdots \ldots \ldots . .$.

Butter-butterfy $\ldots \ldots \ldots$......

Butter-butterfy...$\ldots \ldots 10$

Doctor-linwyer $\ldots \ldots \ldots \ldots$.

Loctor-

..........

. .............

Joy- $\rightarrow$ rrow

Bed-couch...........

Tobrco-cht........

Tobacco-cigarette .....

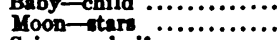

Scissor-knife ..............

Quiet-quilt $\ldots \ldots \ldots \ldots \ldots . .10$

Green—envy $\cdots \ldots \ldots \ldots \ldots$

Street-lane

King-queen

Cheese-cracker ............

Blowom-flower .......

Afraid-courageov ..... 
Case No. 2r33.-M. F. Unclassified reactions, mostly "far fetched" or incoherent; perseveration.
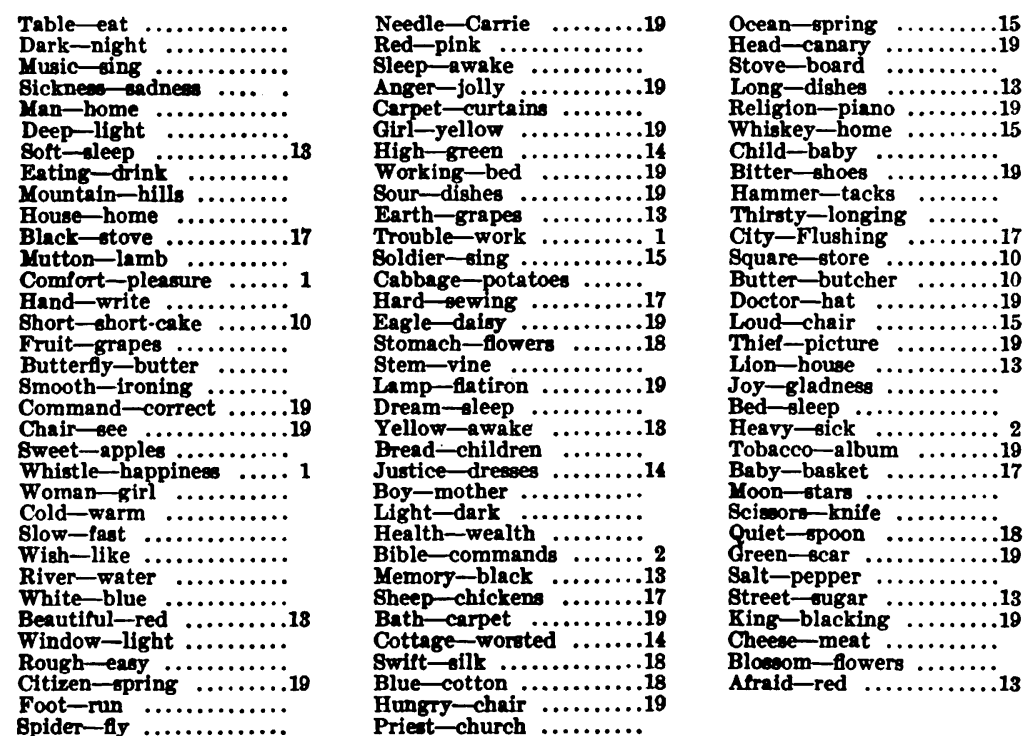

Case No. 4569.-L. K. Marked stereotypy; unclassified reactions, mostly incoherent.
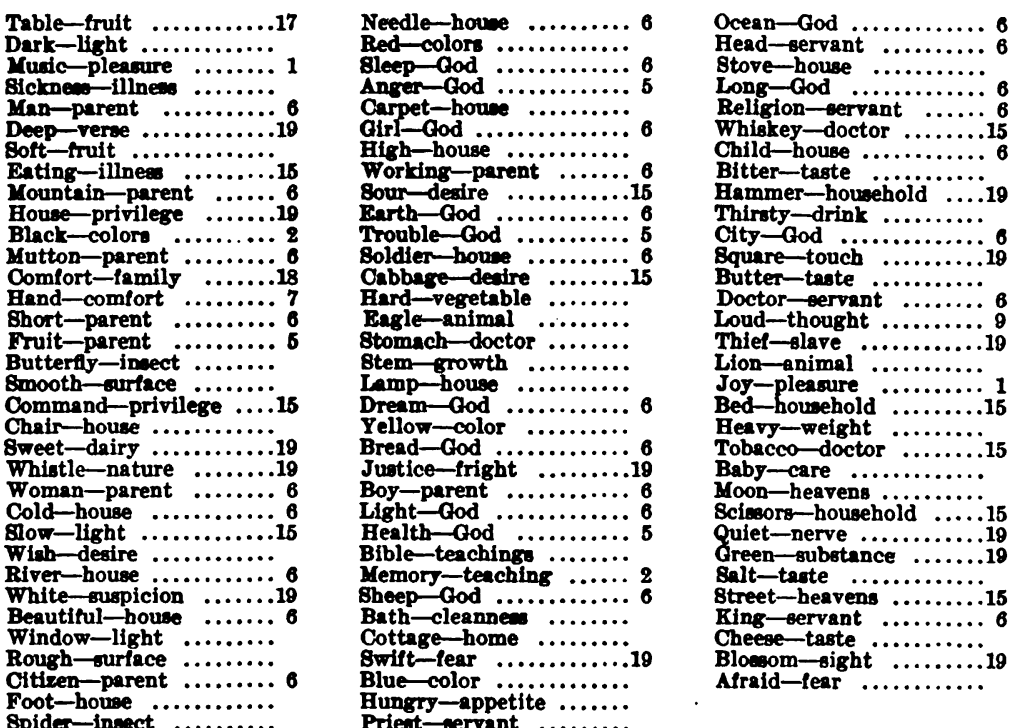

Spider-insect

Prieat-servant .......... 
CASE No. 6164-L. E. Remarkably persistent tendency to give sound reactions; numerous sound neologisms; no reactions given in response to some of the stimulus words on the ground that she had "no word to match."
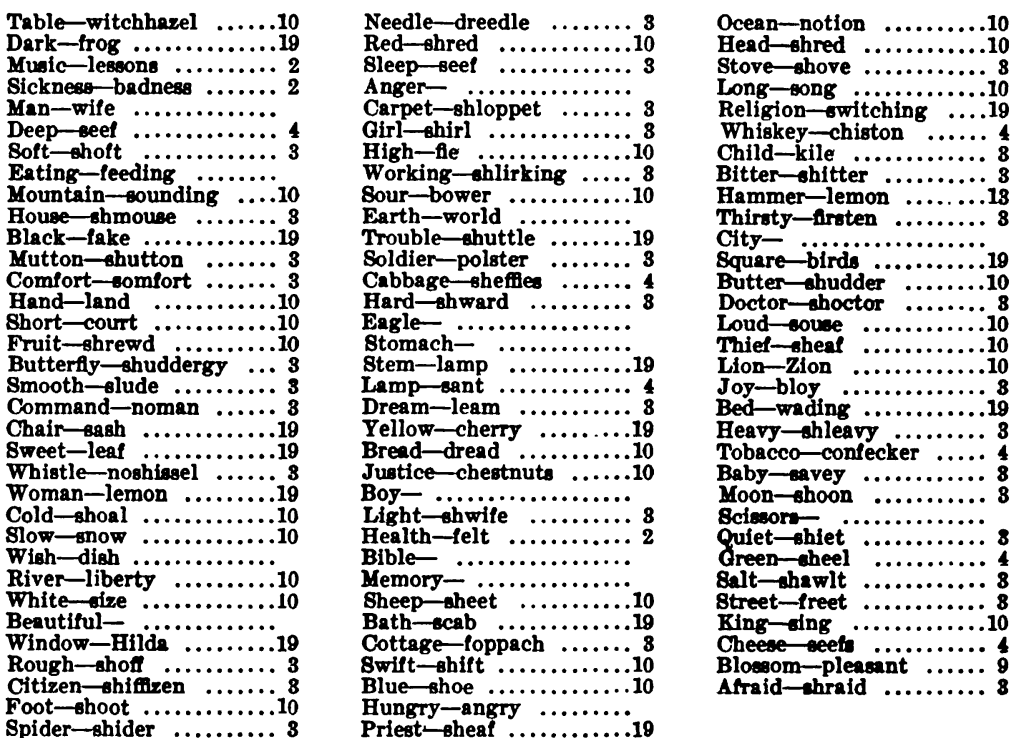

Case No. 3606.-F. W. Neologisms; some particles; many unclassified reactions, mostly incoherent.
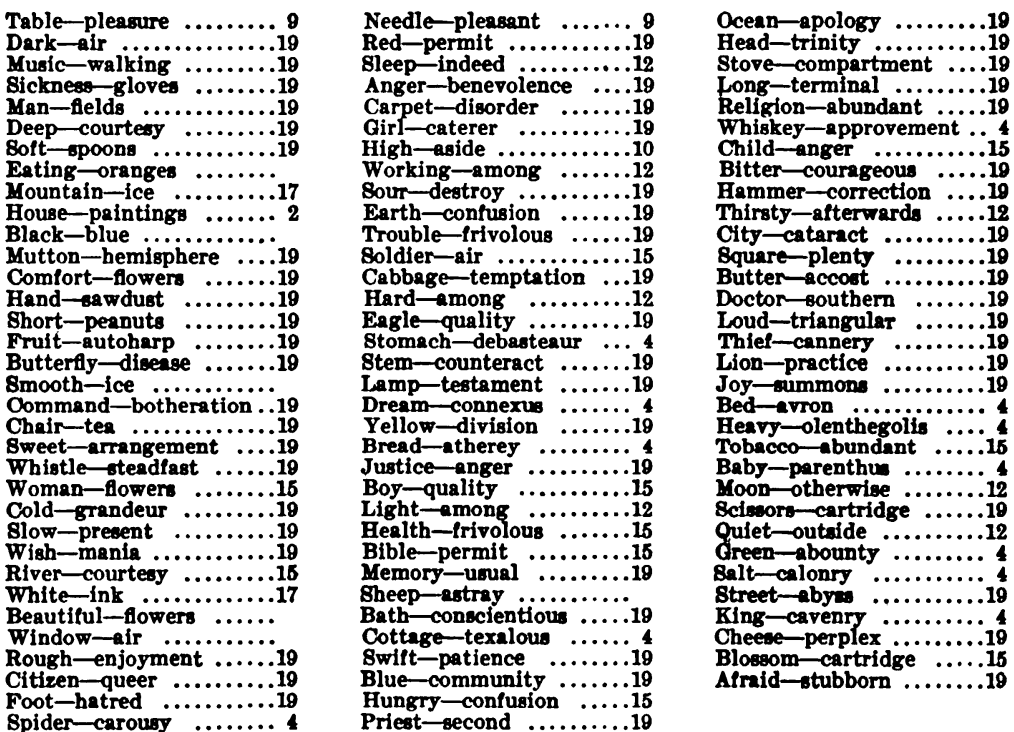
EPILEPSY.

Most of the cases of epilepsy in our collection show advanced dementia and in some the clinical history would indicate also original mental inferiority, that is to say, imbecility or feeblemindedness.

In these cases the dominant characteristic, so far as shown in the test records, seems to be a narrowing of the mental horizon manifested firstly by a tendency to repeat many times one or another word, and secondly by an abnormally pronounced tendency to make use of non-specific reactions or particles of speech. Occasionally other abnormalities are noted, such as perseveration or distraction.

We submit here copies of some test records.

Case No. 5410.-W. T. K. Repetition of words previously given; nonspecific reactions.
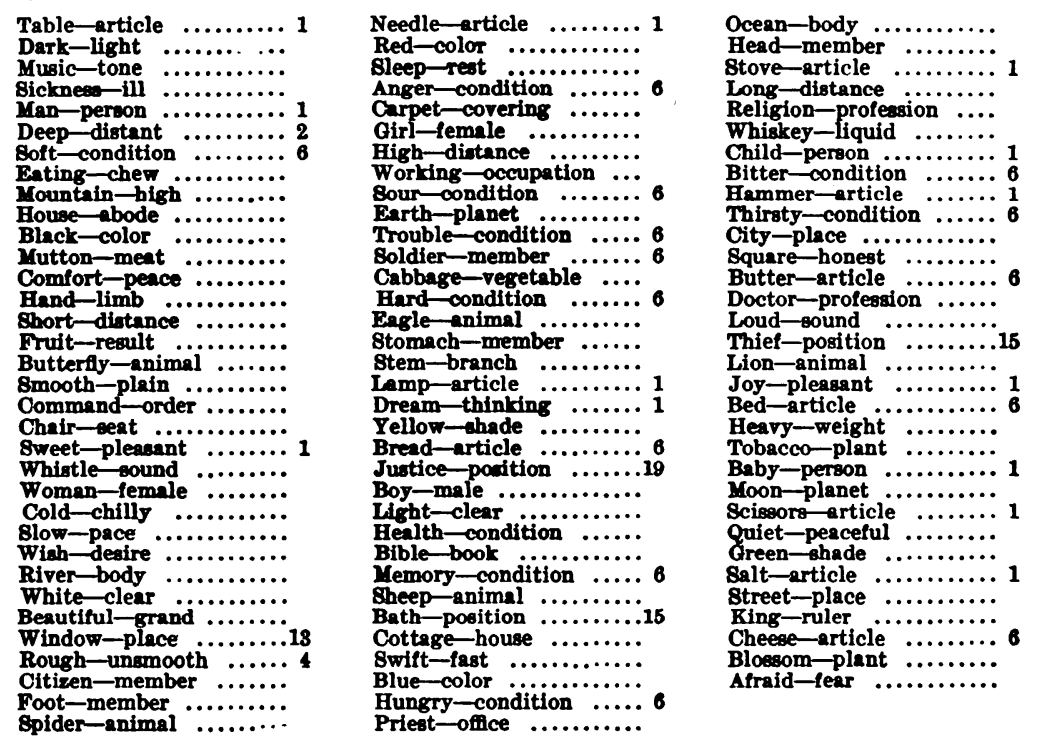
Case No. 2208.-J. A. Repetition of words previously given; nonspecific reactions.
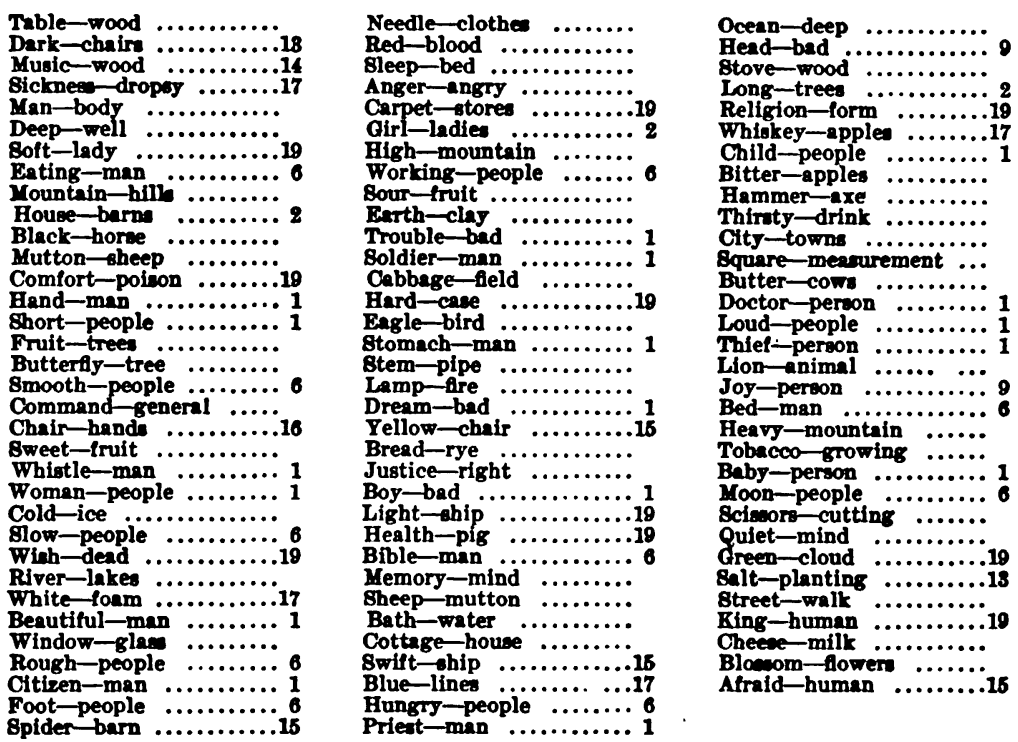

CASE No. or218.-E. M. Repitition of words previously given; nonspecific reactions.
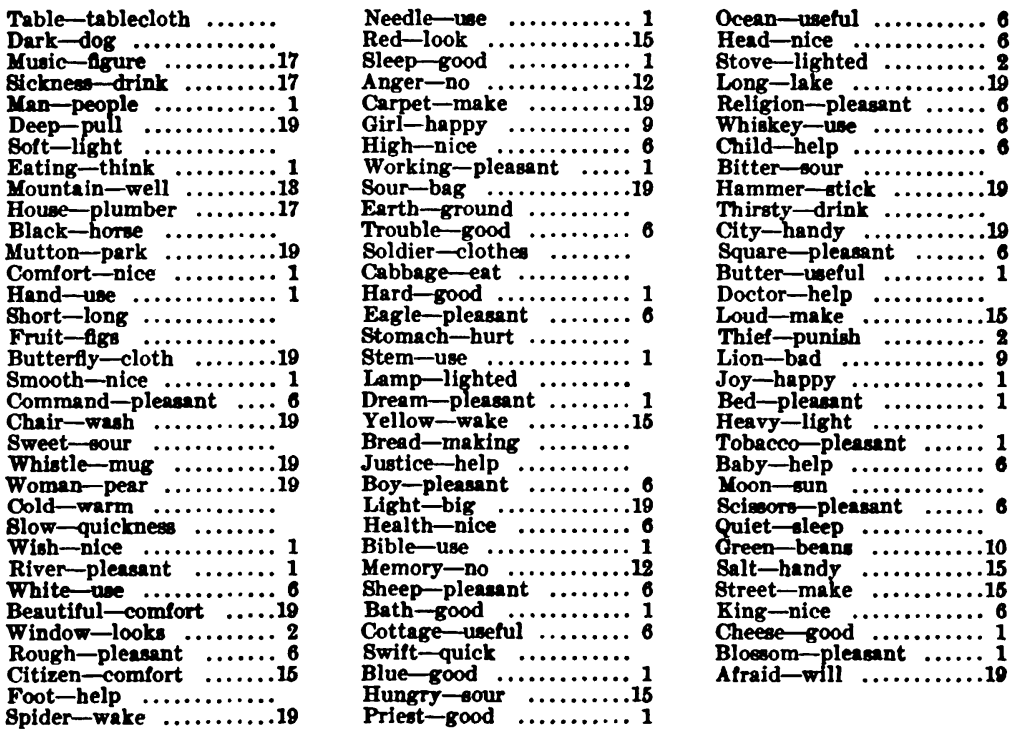
Case No. 4989.-C. H. Repetition of words previously given; nonspecific reactions; particles.
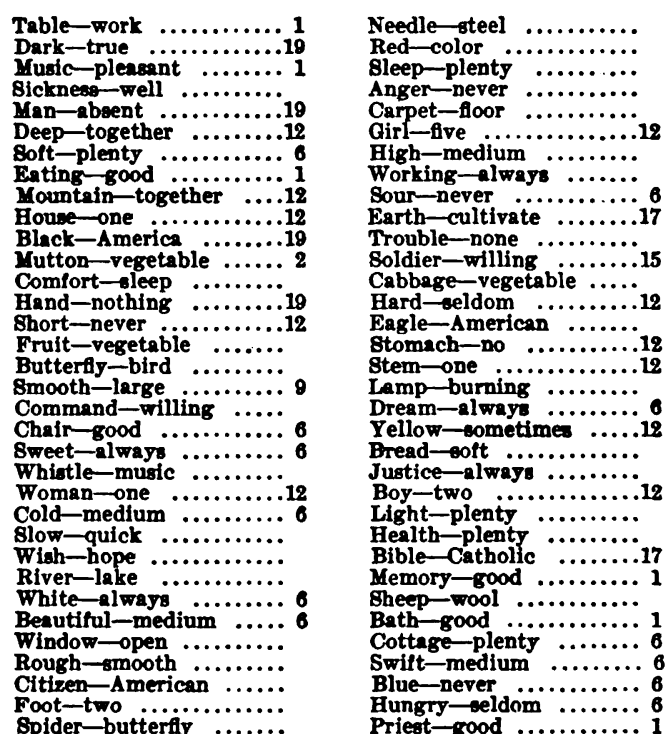

Ocean-three $\quad$..........12

Head-good ............ I

Stove-burning $\quad \ldots \ldots \ldots \ldots$

Religion-willing $\ldots . . . . .15$

Religion-willing $\ldots \ldots \ldots$.

Whiskey $\rightarrow$ bome...$\ldots \ldots \ldots .19$

Child-good $\ldots \ldots \ldots \ldots \ldots$.

Bitter-never

Tammer -tool $\ldots \ldots \ldots \ldots$

Thirsty $\rightarrow$ eldom

City-New York ..........

Square-always ..........

Butter-good $\ldots \ldots \ldots \ldots \ldots)$ 1

Loud-medium $\ldots \ldots \ldots \ldots$.

Loud-medium

Lion-animal $\ldots . . . \ldots \ldots . .$.

Joy-plenty...$\cdots \cdots \cdots \cdots$

Bed-plenty $\ldots \ldots \ldots \ldots \ldots, 6$,

Heavy-medium $\ldots \ldots \ldots .6$.

Tobacco-yes $\ldots \ldots \ldots \ldots .12$

Baby-more

gcienor b ............

Ouiet-plenty.........

green - good $. . . \ldots \ldots \ldots \ldots, 6$

Salt-little $\ldots \ldots \ldots \ldots \ldots \ldots$.

Btreet-lots .............18

King-none $\ldots \ldots \ldots \ldots \ldots \ldots$

Cheese- reldom ..........

Afraid-oometime

Case No. 4847-C. C. Distraction.
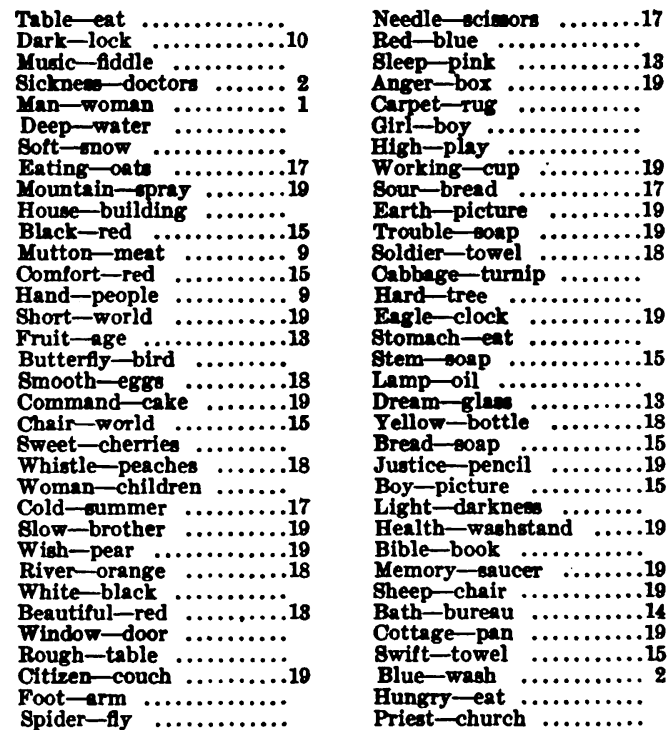

Ocean-beans $\quad . . \ldots \ldots \ldots 19$

.........18

Long-fg . . ................

Religion-church $\ldots \ldots \ldots$.

Whiskey-tea..$\ldots \ldots \ldots \ldots .17$

Child-people $\ldots \ldots \ldots \ldots \ldots$.

Bitter-stomach ........19

Hammer-tack...$\cdots \cdots$

Thirsty-peach...$\cdots \cdots \cdots$

gquare $\rightarrow$ soap $\ldots \ldots \ldots \ldots \ldots 15$

Butter-lard .............

Doctor-sick . ............

Loud-head ...........19

Thief-cup $\ldots \ldots \ldots \ldots \ldots \ldots$

Lion-bottle $\ldots \ldots \ldots \ldots \ldots$.

Joy-pitcher .............19

Bed-sheet $\ldots \ldots \ldots \ldots \ldots$.

Tobacco-mustard .......19

Baby-pepper ............18

Moon-heater ..........1

8cissory-atring $\ldots \ldots \ldots \ldots$.

Quiet-lace...$\ldots \ldots \ldots \ldots$.

Green-red

Salt $\rightarrow$ soda $\ldots . . . \ldots \ldots \ldots \ldots 17$

Street $\rightarrow$ soldier . . . . . . . . .

Cheese-cake $\ldots \ldots \ldots \ldots \ldots$.

Blowom-ahell …............

Afraid-blotter ..........19

Spider-fiy $\cdots \cdots \cdots \cdots \cdots$

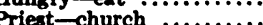


Case No. 3597.-L. T. Some neologisms, all possessing obvious meaning.
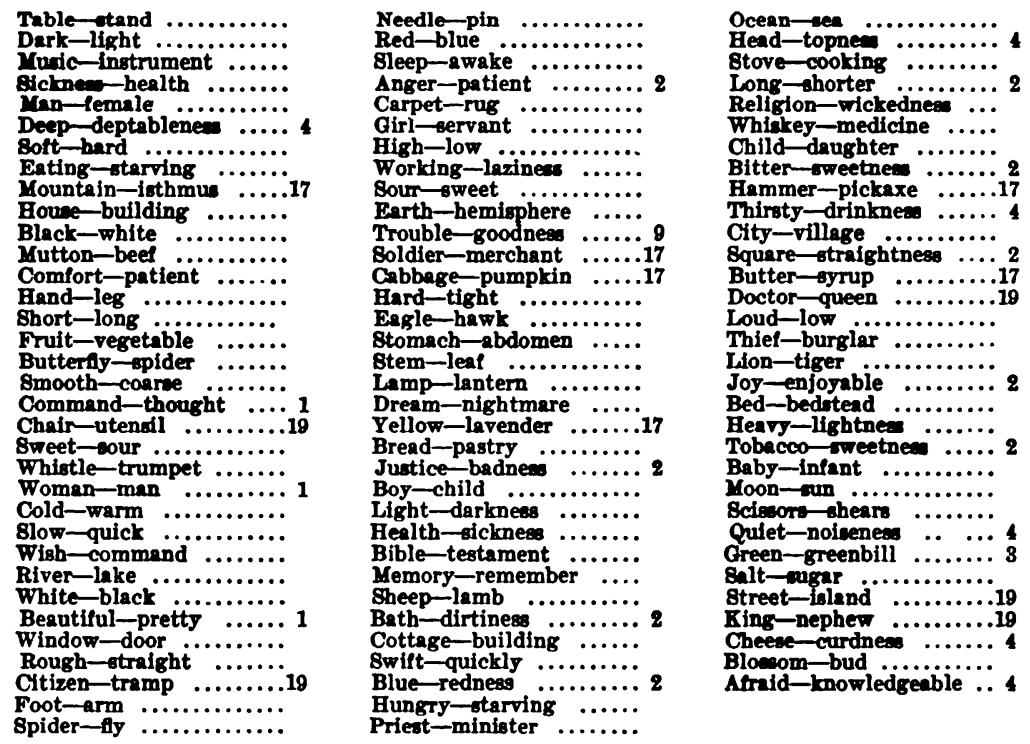

GENERAL PARESIS.

Cases presenting no considerable dementia or confusion and cases in a state of remission are apt to give normal test records. As we proceed from the records of such cases to those of cases showing mental deterioration we observe a gradual reduction in the values of reactions, contraction of the mental horizon, ${ }^{1}$ and the appearance of the phenomenon of perseveration. We submit the following test records for illustration:

\footnotetext{
${ }^{1}$ What we mean by contraction of the mental horizon has already been
} described in connection with epilepsy, page 182 . 
Case No. 4047.-C. A. F. Almost complete remission of all mental symptoms. Normal record.
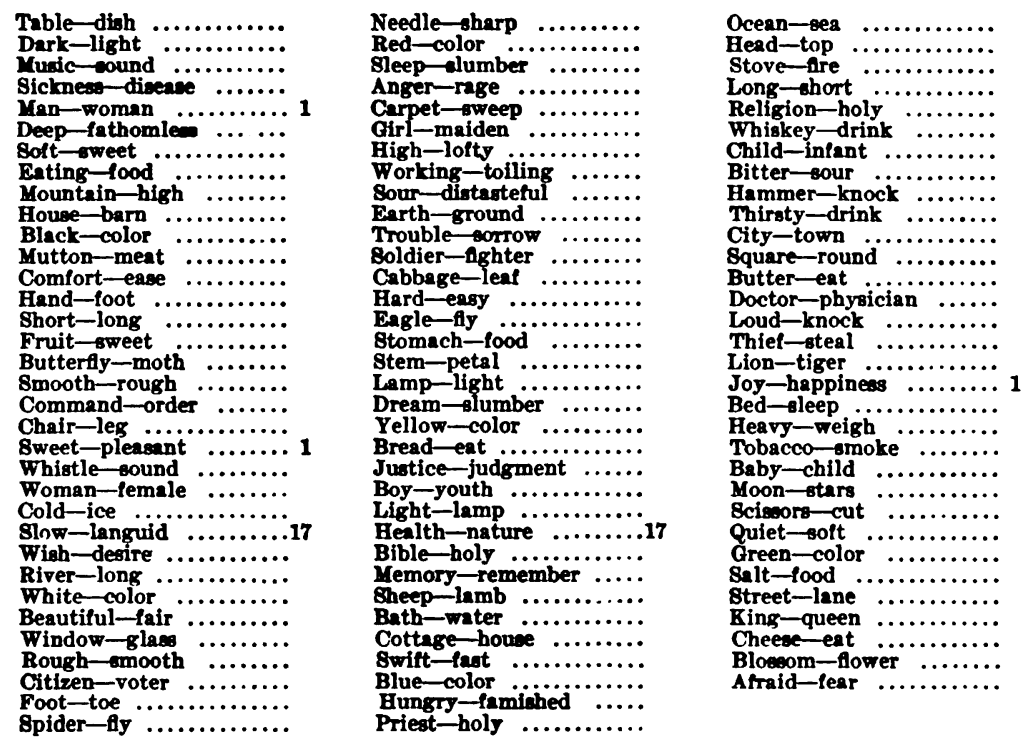

CASE No. 6660.-F. F. Repetition of words previously given; nonspecific reactions; unclassified reactions some of which are "circumstantial" (see page 16).

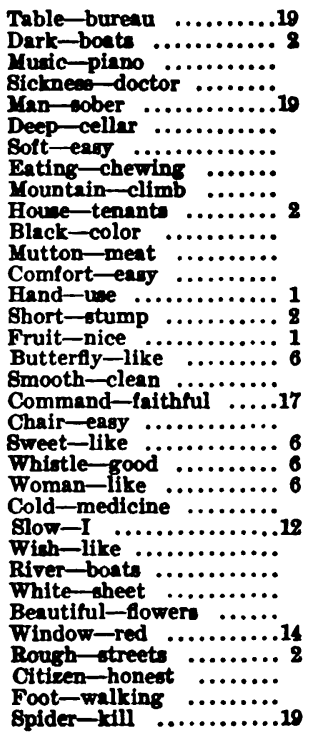

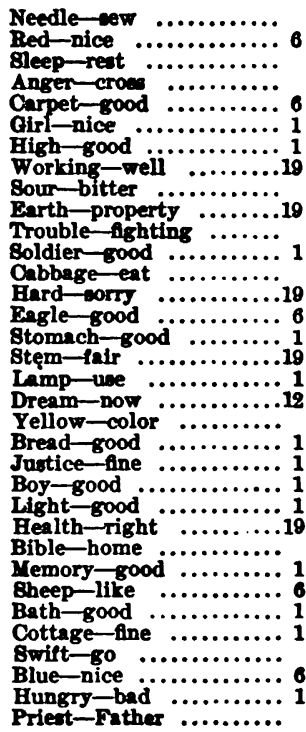

Ocean-boats

Head-brain

Stove-heat

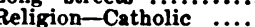

Whiskey-bad ............

Child-good

Bitter-orrow ...........

Hammer-use......... .1

Thirsty-drink

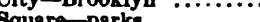

Butter-ice ...............2

Doctor-

Loud-holler $\cdots \cdots \cdots \cdot \ldots$

Thief-no $\ldots \ldots \ldots \ldots \ldots \ldots, 2$

Joy-hope

Bed-rest..........

Heavy-losd

Tobecco-good . ........... 1

Baby-good

Moon-light

Scissors - use .............

Quiet-good ............

Green-nice ..............

Salt-une

Btreet-nice $\ldots \ldots \ldots \ldots \ldots$

King-right $\ldots \ldots \ldots \ldots \ldots$ 19

Bloseom-BTOF ............

Afraid-no ............... 
CASE No. 6185.-R. N. Numerous particles of speech; some unclassified reactions, chiefly "circumstantial" (see page I6).
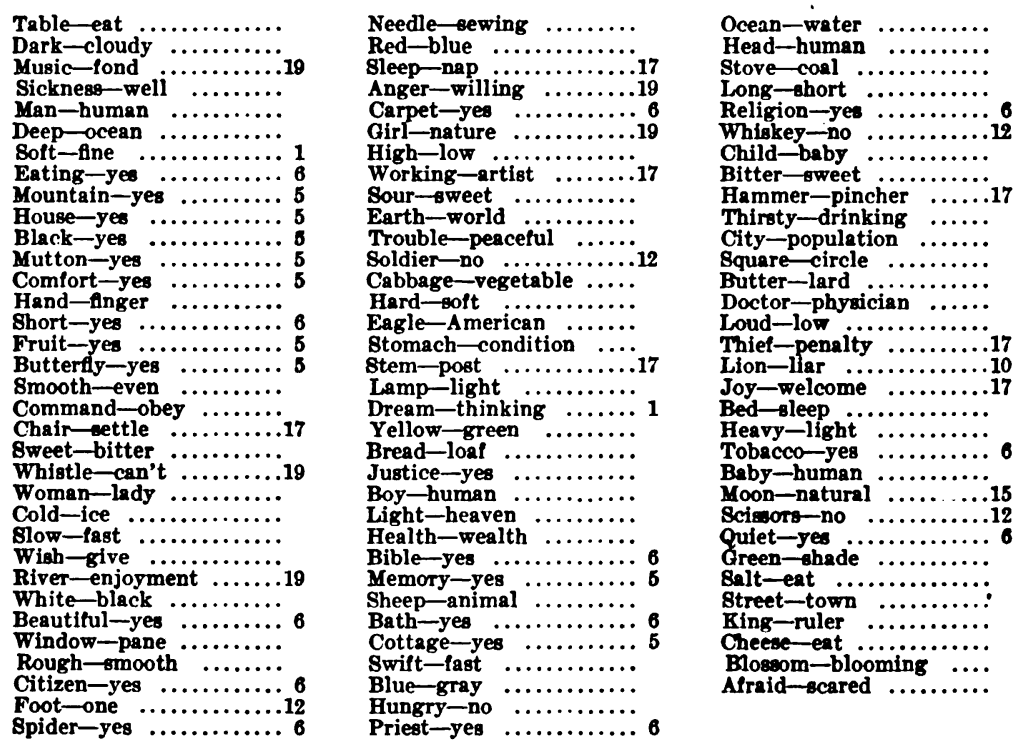

Case No. 6518.-C. Z. Perseveration shown by numerous instances of association to preceding reaction.
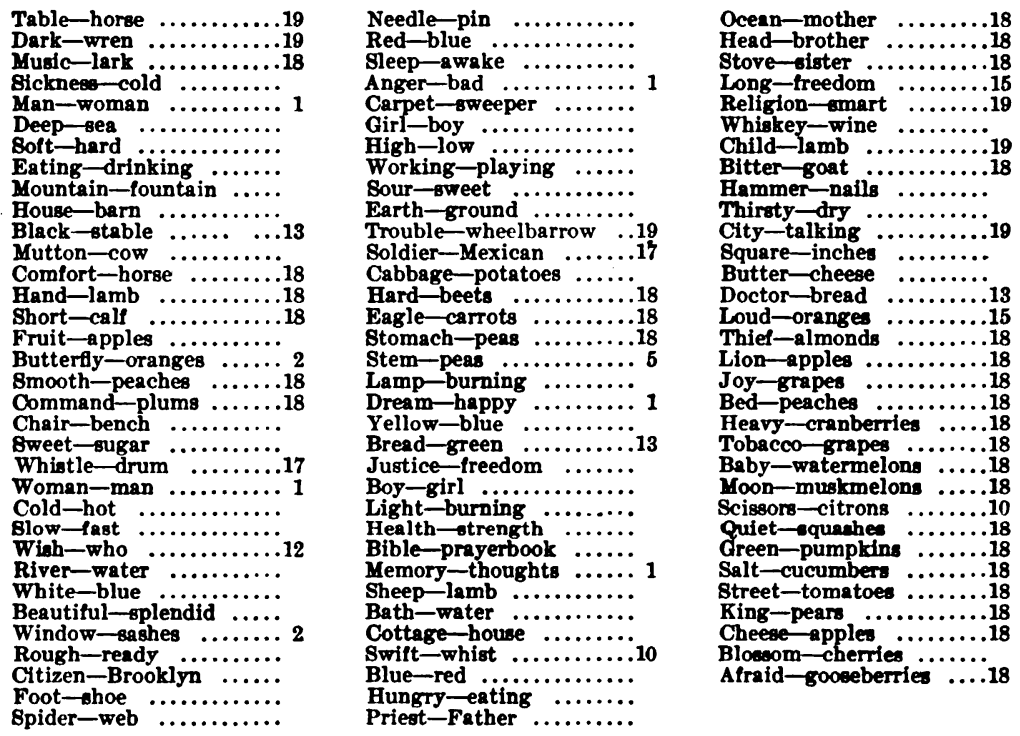
CAse No. 5329.-B. W. Perseveration; record almost entirely made up of instances of association to preceding reaction.
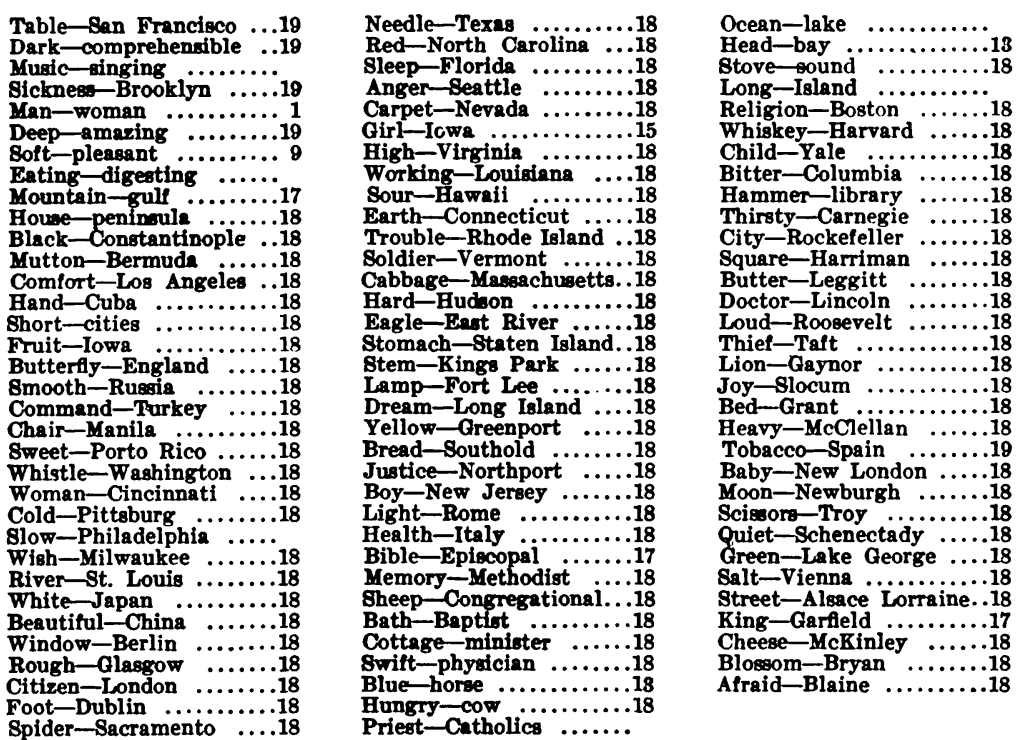

MANIC-DEPRESSIVE INSANITY.

In this disorder the departures from the normal seem to be less pronounced than in the psychoses considered above. The number of individual reactions is in most cases not greatly above the normal average; and, so far as their character is concerned, we find that many of them are classed as normal, in accordance with the appendix to the frequency tables; among the unclassified reactions, which are quite frequent here, we find mostly either obviously normal ones, or some of the type to which we have already referred as "far-fetched," while others among them are "circumstantial" (see p. I6) ; further we find that most of the remaining individual reactions fall into the general group of partial dissociation: non-specific reactions, sound reactions, word complements, and particles.

In some cases the only abnormality that is found is that of an undue tendency to respond by non-specific reactions, most of them being common and there being no excessive number of individual reactions. It would seem legitimate to assume that this tendency 
is here to be regarded as a manifestation of the phenomenon which is clinically described as dearth of ideas. It is significant that this tendency is observed not only in depressive phases of the psychosis, but also in manic phases and even in the normal intervals of recurrent cases or after apparent recovery in acute cases; this will be seen from some of the test records which are here reproduced.

Occasionally cases are met with which give a large number of unclassified reactions, seemingly incoherent. There can be no doubt that at least some of these cases are clinically perfectly typical ones of manic-depressive insanity, yet the test records strongly resemble, in some respects, those of dementia præcox. Since clinically the distinction between typical cases of these psychoses can be so clearly made on the basis of the disorders of the flow of thought respectively characterizing them, it could hardly be assumed that the associational disturbances in these two groups of cases are truly related, although there may be an apparent resemblance; it must be acknowledged that we are here confronted with one of the most serious shortcomings of the association test, or at least of the present method of applying it.

Case No. 5236.-M. B. Depressive attack. Normal record.
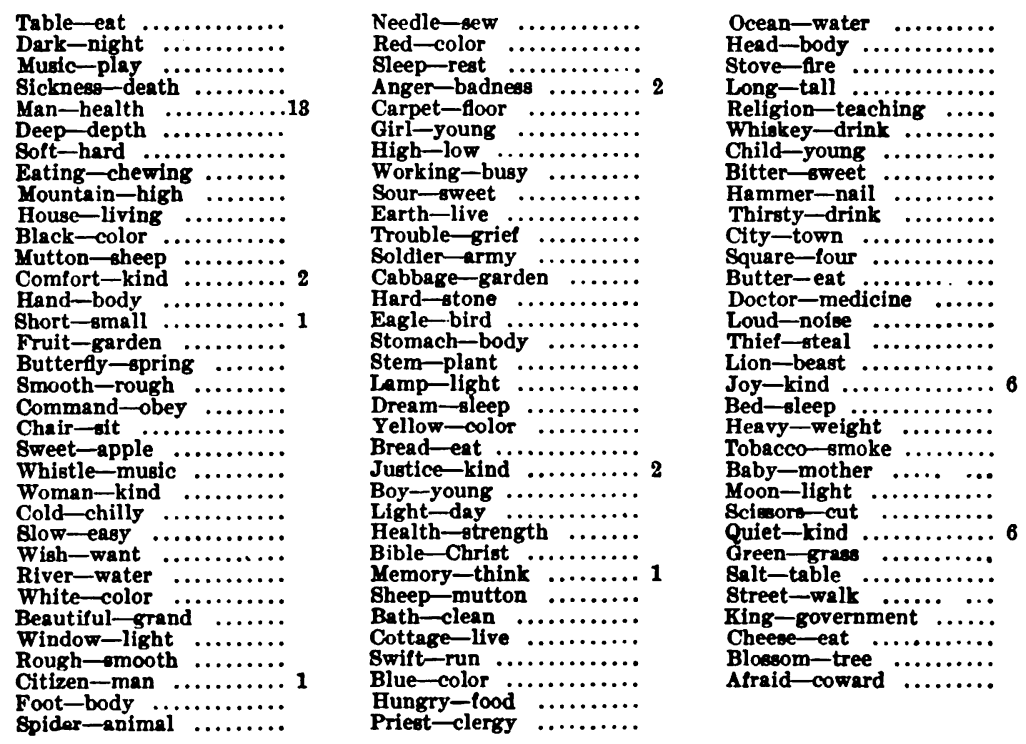
[Oct.

CAse No. 6r20.-M. L. Maniacal attack. Fifteen individual reactions, of which II are classed as normal in accordance with the appendix to the frequency tables.
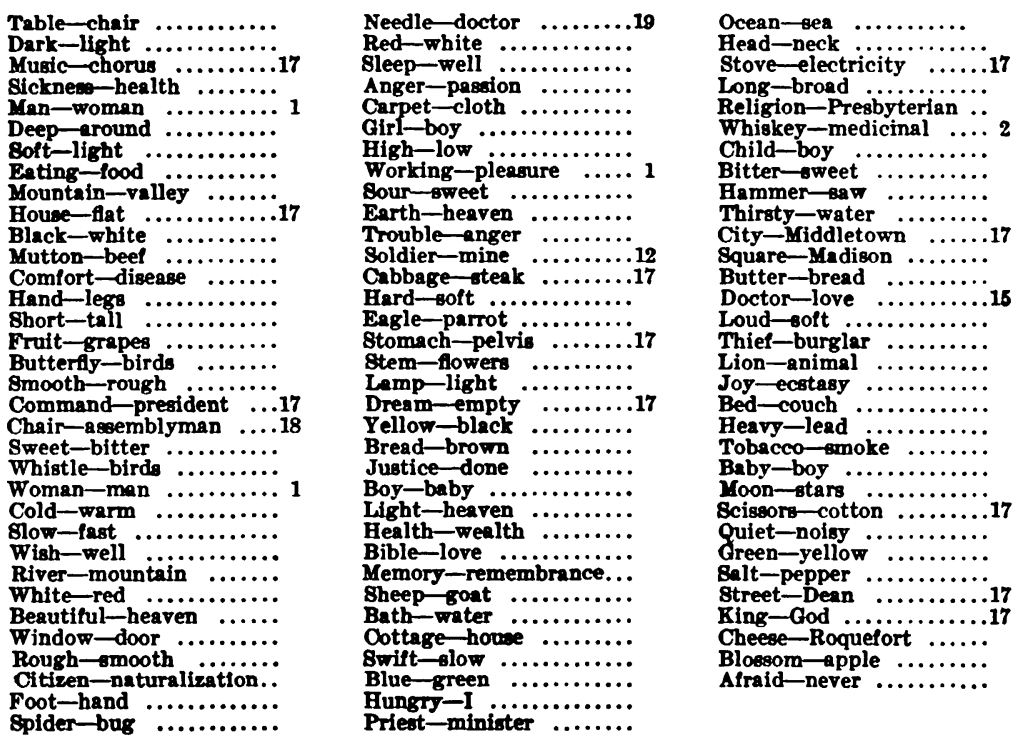

Case No. 6367.-J. N. Depressive attack. Only two individual reactions, both classed as normal; undue tendency to give non-specific reactions.
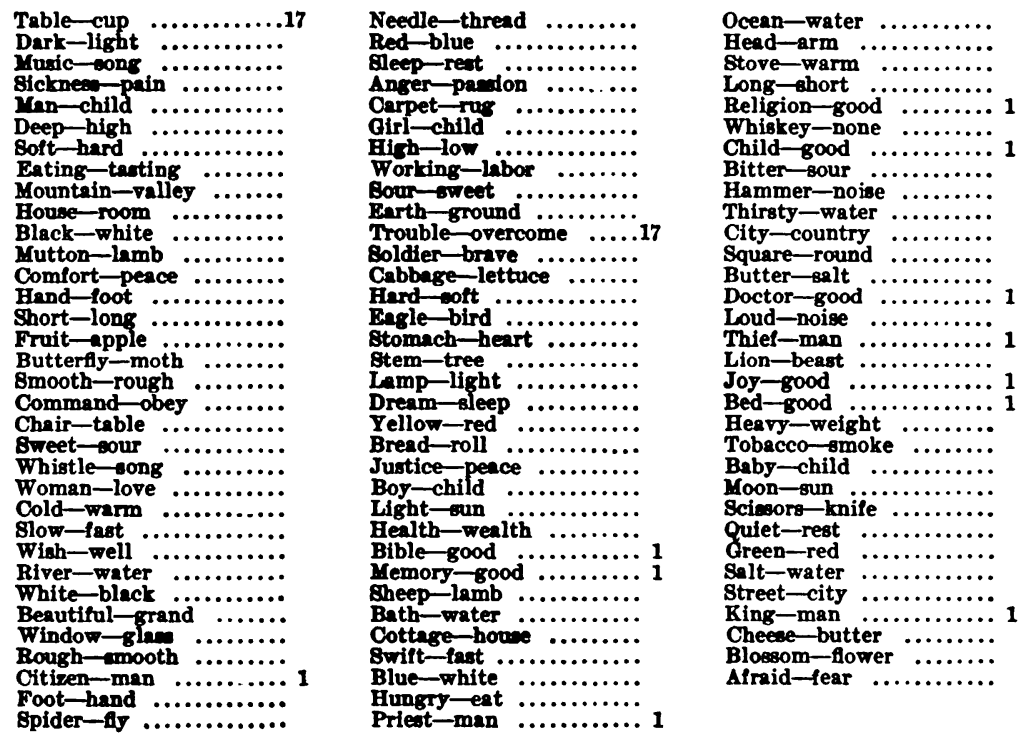
CASE No. 5162.-W. H. Recurrent attacks, mixed in character; at time of test patient was in a normal interval. 5 individual reactions, of which $I$ is classed as normal, $I$ as a derivative, 2 as non-specific, and $I$ as a sound reaction; undue tendency to give non-specific (common) reactions.
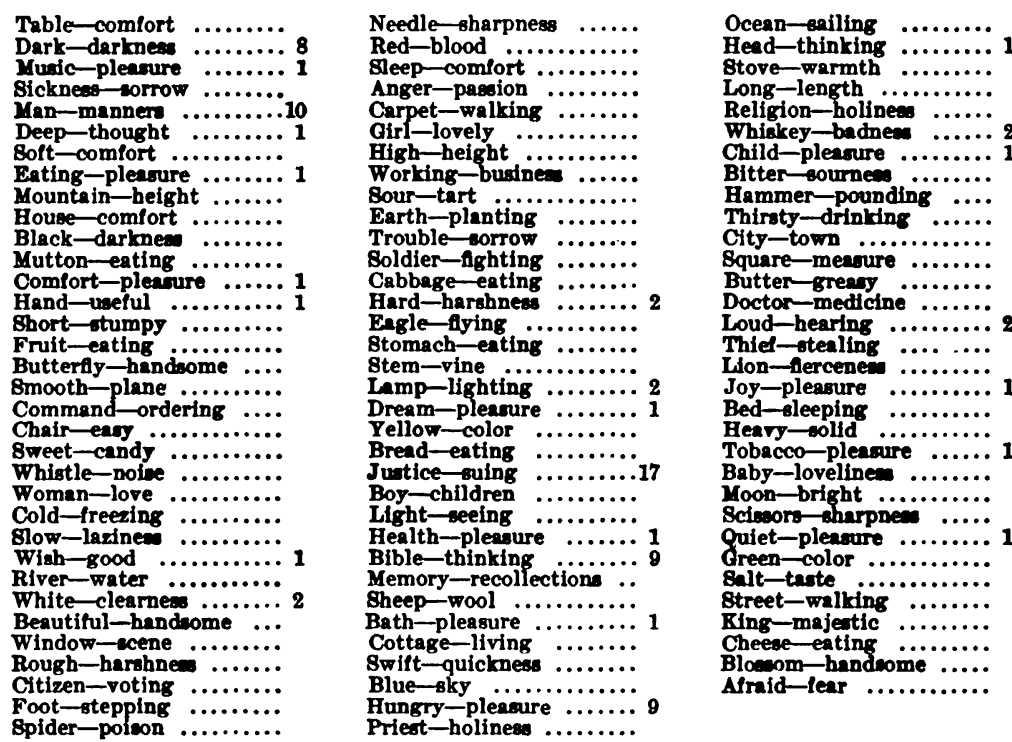

CASE No. 6279.-A. F. Maniacal attack; at time of test patient had improved, though not recovered. Non-specific reactions; particles. (Patient does not speak English with perfect fluency.)

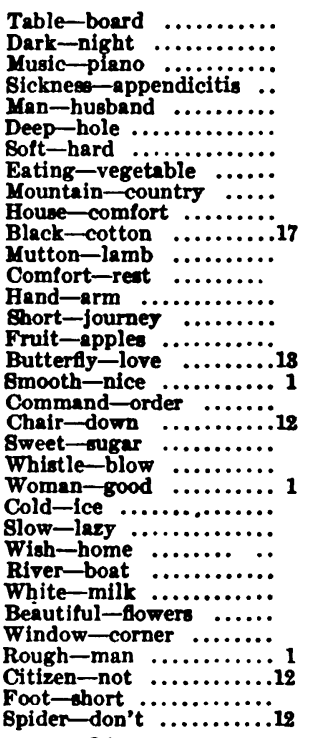

24
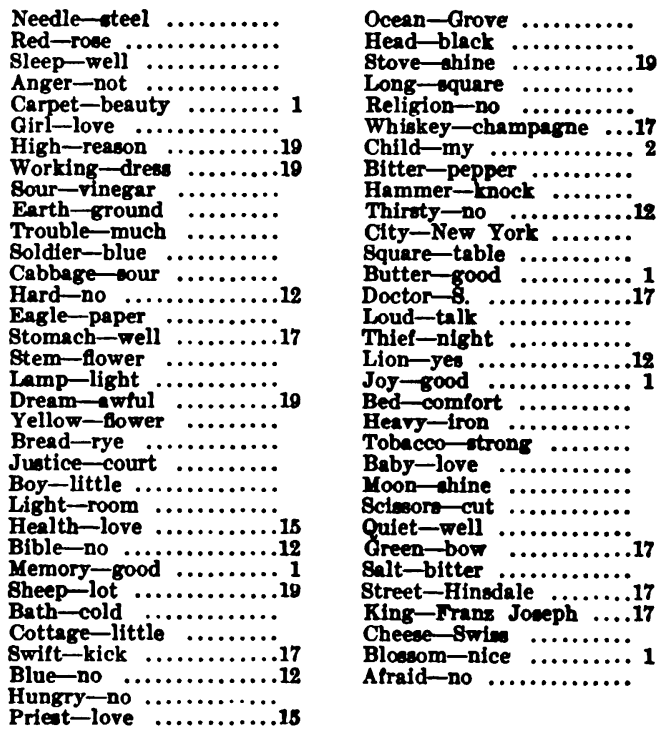
Case No. 4578.-E. M. Circular insanity of over twenty years' standing; at time of test patient was in a manic phase. Non-specific reactions; doubtful reactions; neologisms, all possessing obvious meaning.
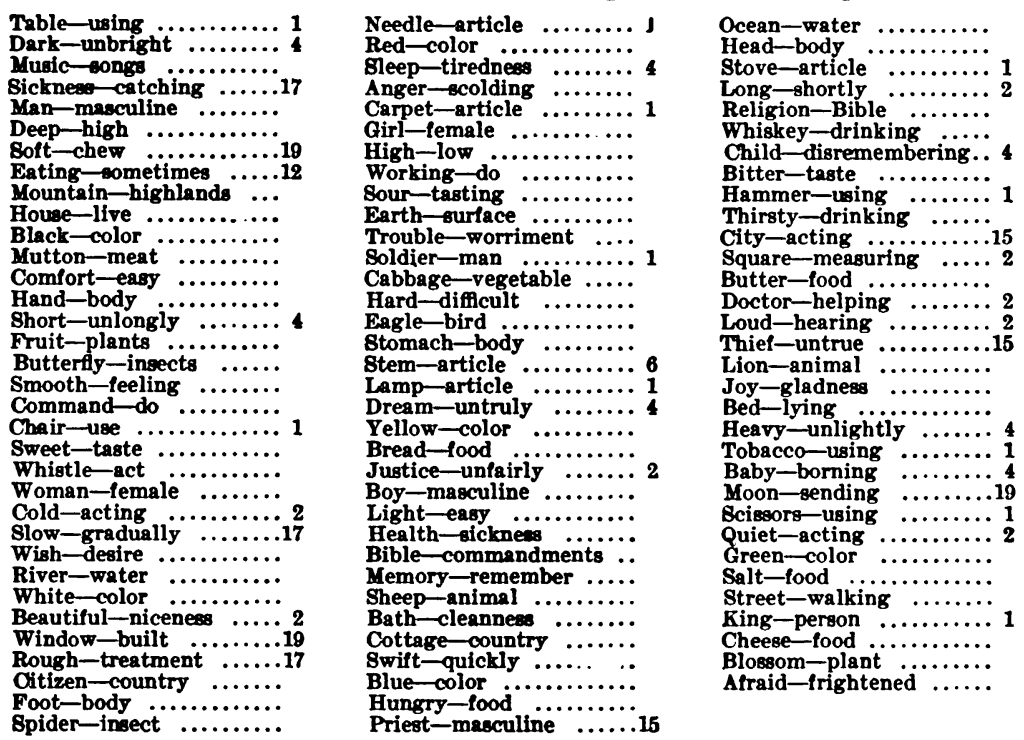

CASE No. 5878.-A. B. Maniacal attack. 48 individual reactions, of which 18 are classed as normal, 1o are sound reactions (2 sound neologisms), I word complement, 8 particles, and II unclassified reactions most of which are either obviously normal or "far fetched" but not strictly incoherent.
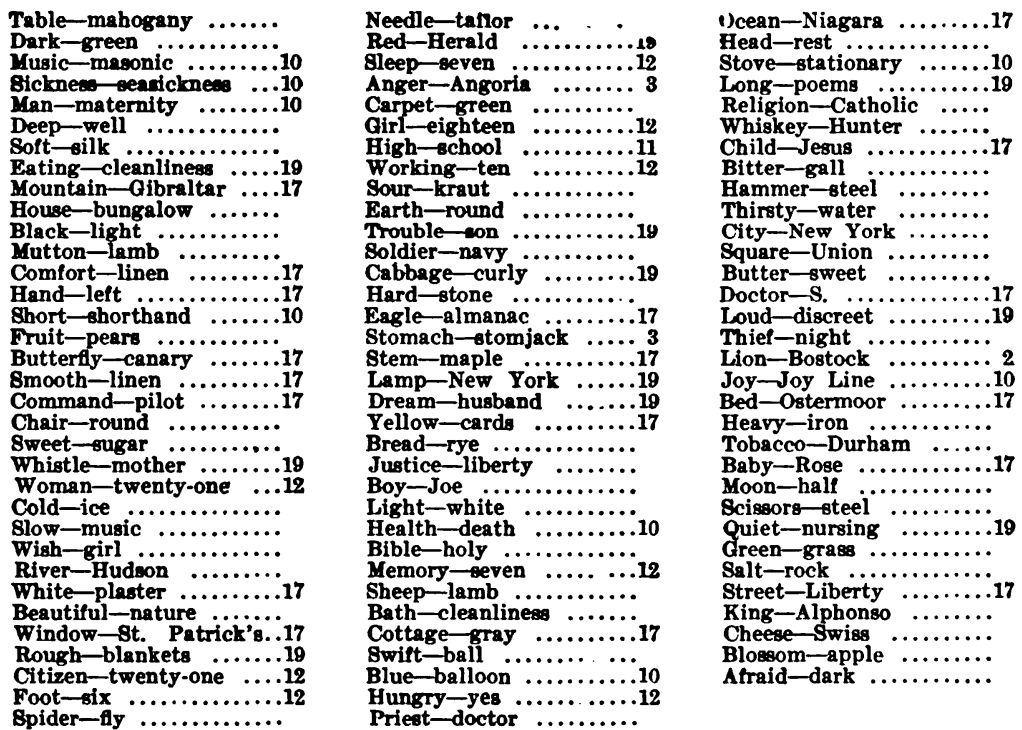
Case No. 6511.-U. B. Maniacal attack. Persistent use of particles oh, me, $I$, none, etc.
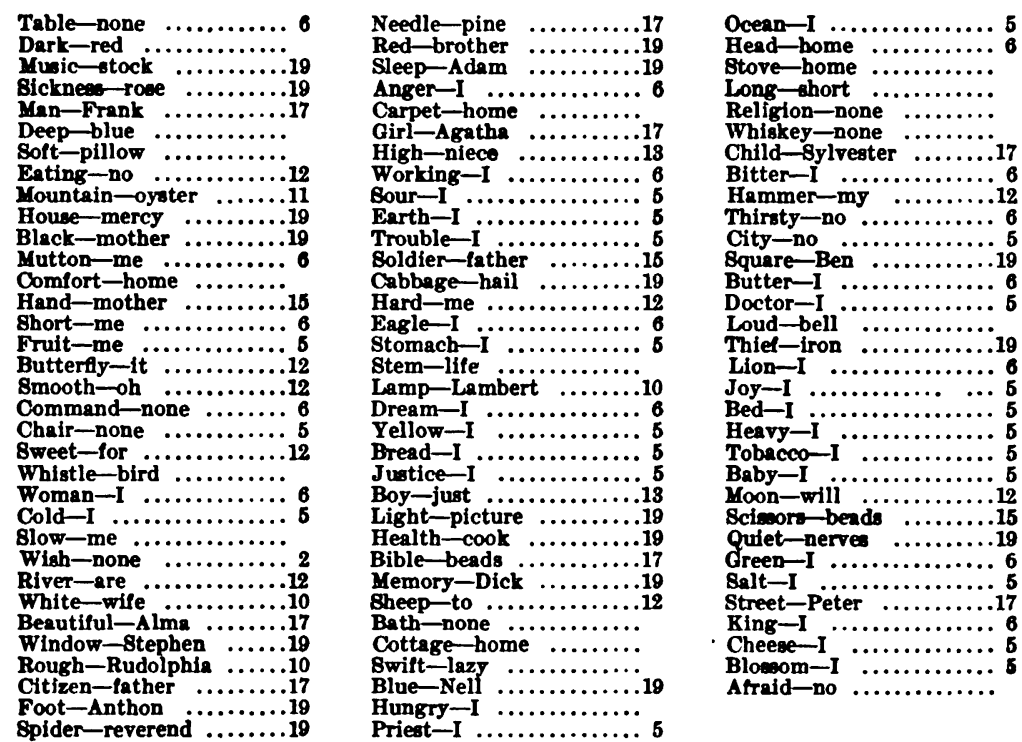

CASE No. 4427.-A. R. Maniacal attack. Unusual number of doubtful reactions; 46 individual reactions of which 9 are classed as normal; 29 are unclassified, some seemingly incoherent.
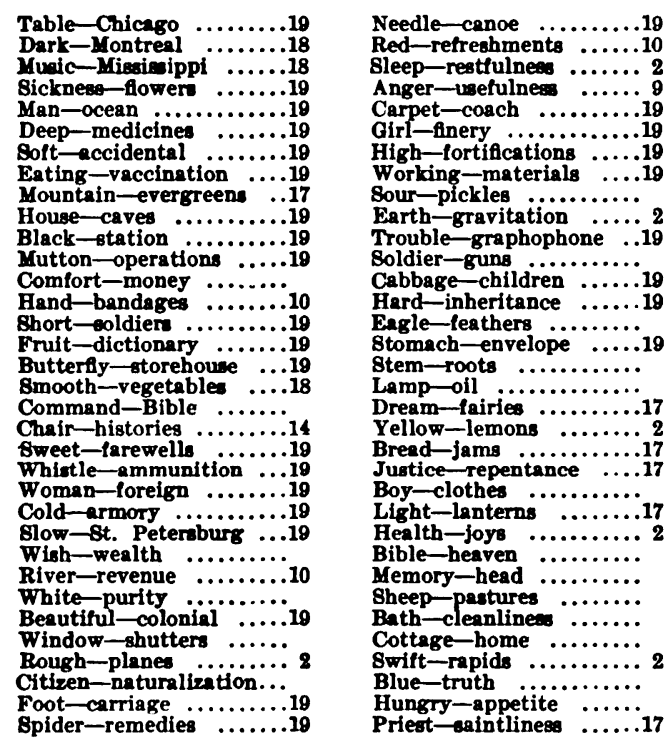

Ocean-ahipe $\ldots \ldots \ldots \ldots$.

Head-intelligence $\ldots . .$.

Stove-woods $\ldots \ldots \ldots \ldots \ldots 2$

Long-trains $\ldots \ldots \ldots . . .22$

Whiskey-drunkennes ...

Child-joyfulnes $\ldots . . .28$

Bitter-olives

Hammer-nuts...$\ldots \ldots$.

Thirsty-water

Square-monument $\ldots . . .2$.

Square-monuments $\ldots \ldots 2$
Butter-crackers $\ldots . . . .17$

Doctor-medicines .......

Thief-detectives..$\ldots \ldots \ldots$. 2

Tion-cetectives..$\ldots \ldots \ldots$ 2

Joy-home $\ldots . . . \ldots \ldots \ldots . .$.

Bed-restrulnew $\ldots . . . . . .2$

Tobacco-cicam .......

Baby-carriege …......

Moon-light

...........

Quiet-pencefulnes ..........

Green-vegetables .......

Salt-wnter ...............

Street-stone

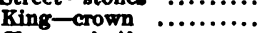

Cheese-knife ….........

Blossom-plants ........2

Spider - remedies...... .19

Priest-aintliness $\ldots . . .17$ 
Case No. 6457.-C. G. Depressive attack. 36 individual reactions of which 9 are classed as normal and 20 as unclassified; among the latter several seem to be incoherent.
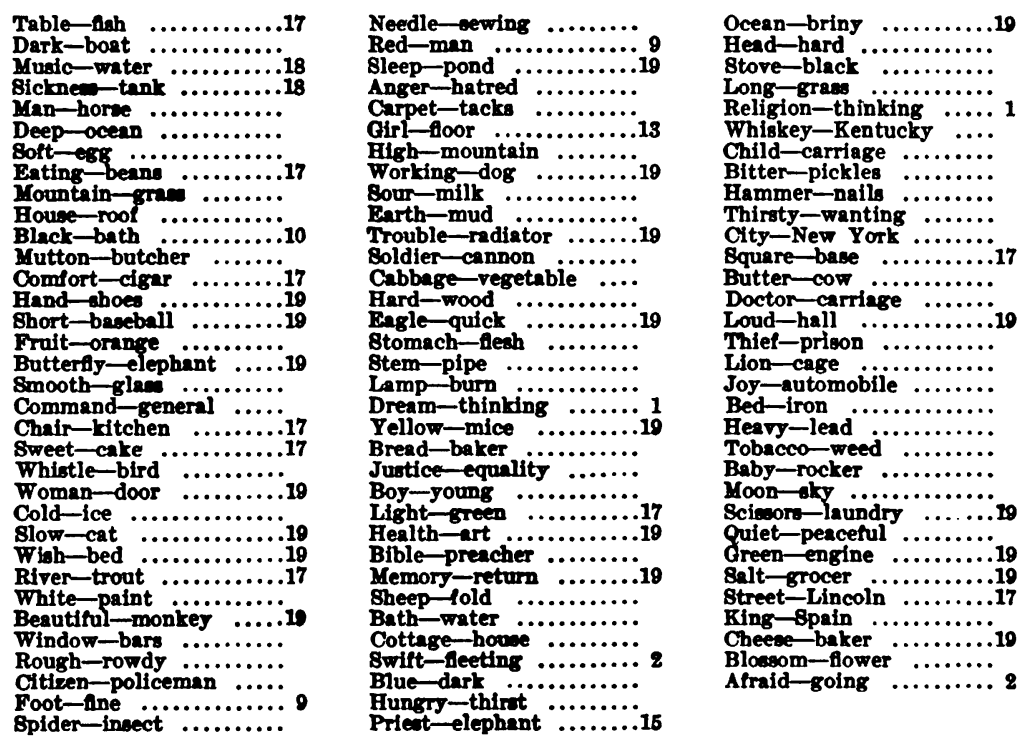

inVolutional MELANCHOLIA; ALCOHOLIC DEMENTIA; SENILE DEMENTIA.

There are so few cases of these psychoses in our series that we can say but little concerning their associational disorders.

In Table $\mathrm{V}$. we show all the types of reactions given by each subject.

We have not observed in our cases of involutional melancholia any undue tendency to give individual reactions. The records are either perfectly normal or slightly abnormal in that they show an increase of the non-specific (common) reactions. In this respect they resemble strongly the records obtained from some cases of manic-depressive insanity. This similarity is of interest in connection with other evidence, recently brought to light, ${ }^{1}$ showing that involutional melancholia is closely related to manic-depressive insanity, if not identical with it.

${ }^{1}$ G. L. Dreyfus. Die Melancholie ein Zustandsbild des manisch-depressiven Irreseins. 1907. 
I9IO] GRACE HELEN KENT AND A. J. ROSANOFF 36I

\begin{tabular}{|c|c|c|c|c|c|}
\hline \multirow{4}{*}{ 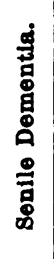 } & B8L9 ON COSD & 5 & : & 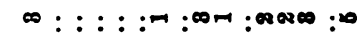 & 8 \\
\hline & 'geso ON ON80 & $88:$ & $:$ & $\infty \sim:::::::::::^{\infty}::^{\infty}$ & $\infty$ \\
\hline & 2E89 ON 0080 & $E^{\infty}$ & $\bullet$ & 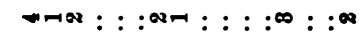 & 20 \\
\hline & . Us? ON & 종 & $:$ & $\infty:-\infty:::^{-1}:::::^{\infty}::^{\infty}$ & ga \\
\hline \multirow{6}{*}{$\begin{array}{l}\bar{\Xi} \\
\text { : } \\
\stackrel{0}{0} \\
\circ \\
\frac{0}{\circ} \\
\frac{0}{8}\end{array}$} & $8[\%$ ON OB8D & $\$ \infty$ & - & $\Xi::::: \infty::::$ : $:$ : & 8 \\
\hline & $600100^{\circ} \mathrm{N}$ O8EO & $\infty$ & $\infty$ & $\infty:::::::::::^{-\infty}: \infty$ & $\approx$ \\
\hline & .0010 ON 0080 & $E^{\infty}$ & $\infty$ & $*::::::::::::^{*}::^{*}$ & 9 \\
\hline & $2009 \cdot 0 \mathrm{~N} 0880$ & 응. & $\infty$ & $\infty:::::::^{\infty}::::::^{\infty}: \infty$ & 은 \\
\hline & 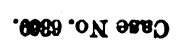 & $8=$ & $\infty$ & $*::::::::::::::^{\infty}::^{\infty}$ & $\infty$ \\
\hline & $800 \%$ ON & $\infty^{\infty} \cdot$ & $\leadsto$ & $\bullet::::::::::::^{\prime}:::$ : & $\bullet$ \\
\hline \multirow{8}{*}{ 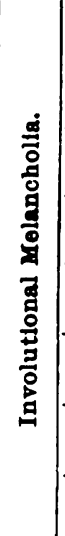 } & $9899 \cdot$ ON 9800 & 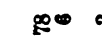 & $\infty$ & $\bullet::::: \mapsto::::::::$ : : & $\infty$ \\
\hline & "6t8t ON อ880 & $\infty^{\circ}$ & $\infty$ & 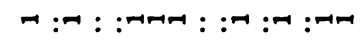 & - \\
\hline & $W 00 \cdot 0 \mathrm{~N}$ O880 & $8 * 0$ & $\infty$ & $\infty::$ : : : : : : : : : : : : & $\infty$ \\
\hline & ON ONOD & $\$ \infty$ & $\neg$ & $::::::: \sqcap:::::^{\infty}: \infty$ & - \\
\hline & $\cdot 0860^{\circ}$ ON & \&્1 & - & 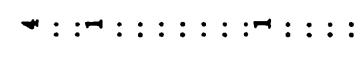 & 0 \\
\hline & 6LQ - ON OBBD & 8 웍 & $*$ & $\neg::::::::::::::$ & $\infty$ \\
\hline & $8181 \cdot 0 \mathrm{~N}$ e810 & $\mathbb{g}^{\infty}$ & $\infty$ & $\propto:::::::::::::::$ : & - \\
\hline & $2030 \cdot 0 \mathrm{~N} 0880$ & $\$ \infty$ & 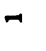 & $::::::::::::::::::$ & $Q$ \\
\hline & 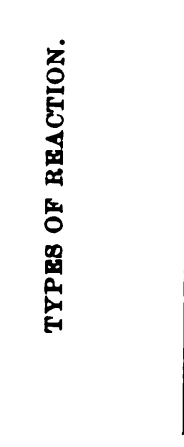 & 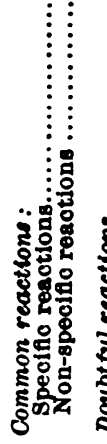 & 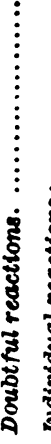 & 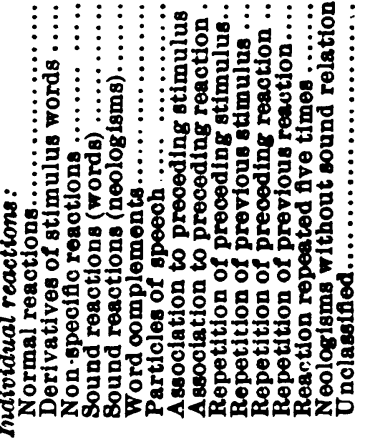 & 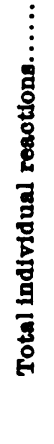 \\
\hline
\end{tabular}


CASE No. 4818.-S. M. Normal record.
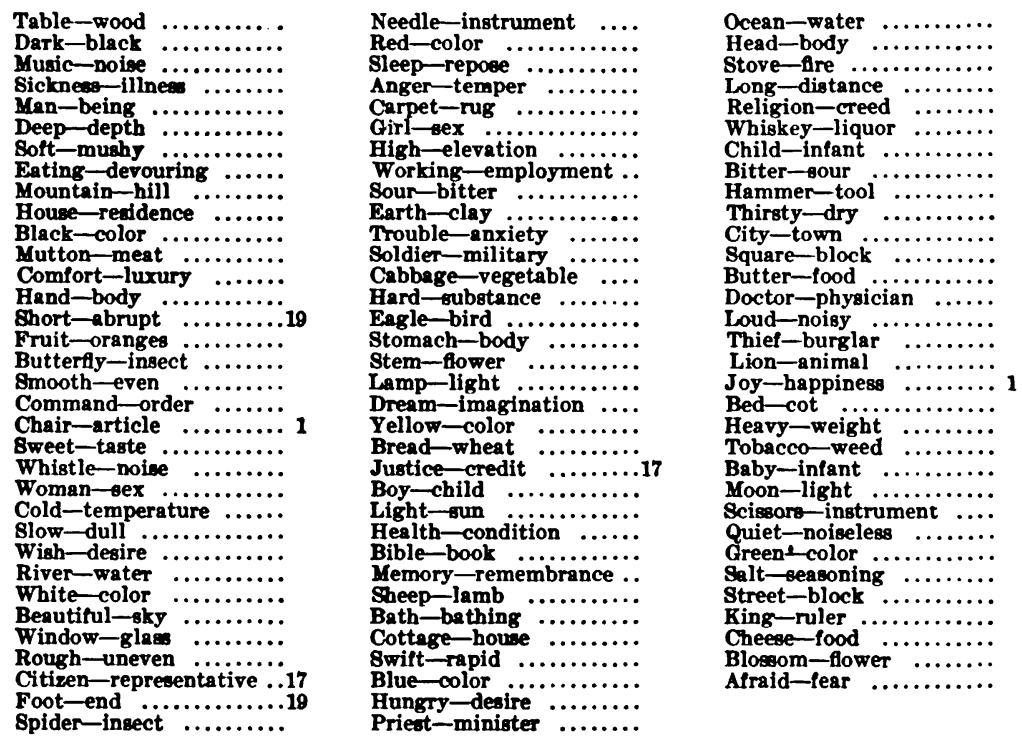

CASE No. 6207.-T. S. No individual reactions; I3 non-specific reactions.
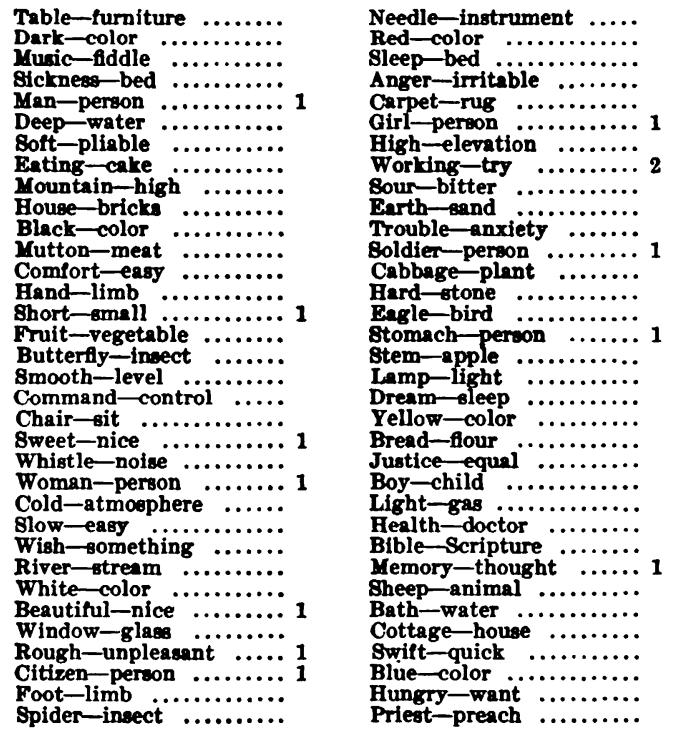

Ocean-water $\ldots \ldots \ldots \ldots$.

Stong-length ..........

Religion-beliet $\ldots . . . \cdots$

Whiskey-drink

Child-person

Bitter pour

Hammer-tool

Thirsty-dry ...................

Thirsty-dry

Square-shape $\ldots \ldots \ldots \ldots$.

Butter-eat

Doctor-physician $\ldots . . . . .$.

Doctor-physic

Thief $\rightarrow$ teal

Joy-glad

Bed-sleep

Teaveco 0 plant........

Baby-child

Moon-light

Scissors-tool

Quiet-rest

..............

Salt-opice

King-ruler $\ldots \ldots \ldots \ldots .$.

Cheese-eat..........

Blossom-flower ........

Spider-insect $\ldots . . . . . .$.

Blue-color .

Priest-preach

Afraid-hide 
CASE No. 5719.-A. W. S. 5 individual reactions; 12 non-specific reactions.
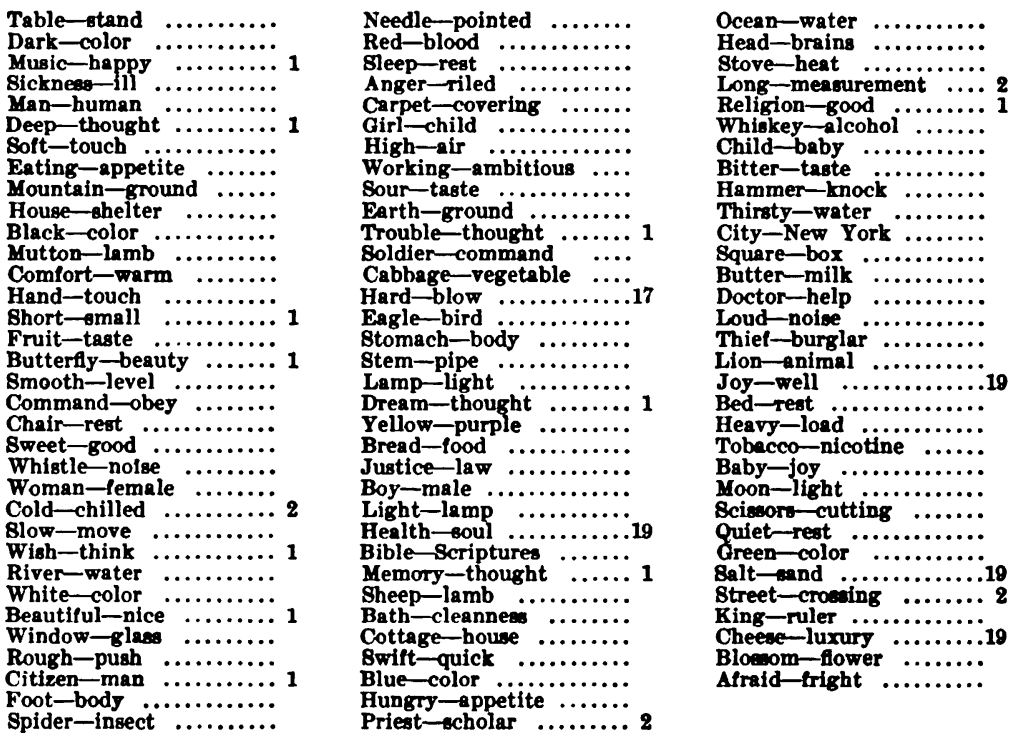

CASE No. 5635. J. D. 13 individual reactions, of which 6 are classed as normal, I particle, 6 unclassified, mostly obviously normal.
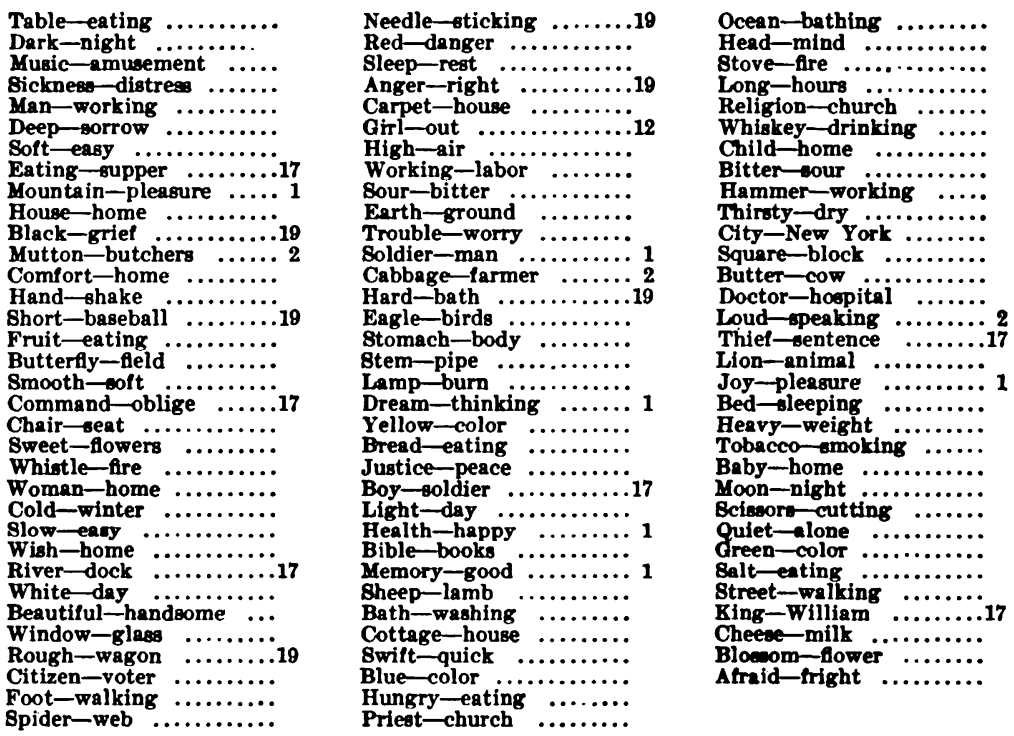

Our cases of alcoholic dementia are clinically without evidences of disturbance of flow of thought. The dementia consists mainly 
in impairment or loss of the power of retention, with resulting amnesia for recent occurrences, and temporal disorientation. The records are either normal or show but slight departures from normal.

Case No. 6369.-J. S. Slight deterioration.
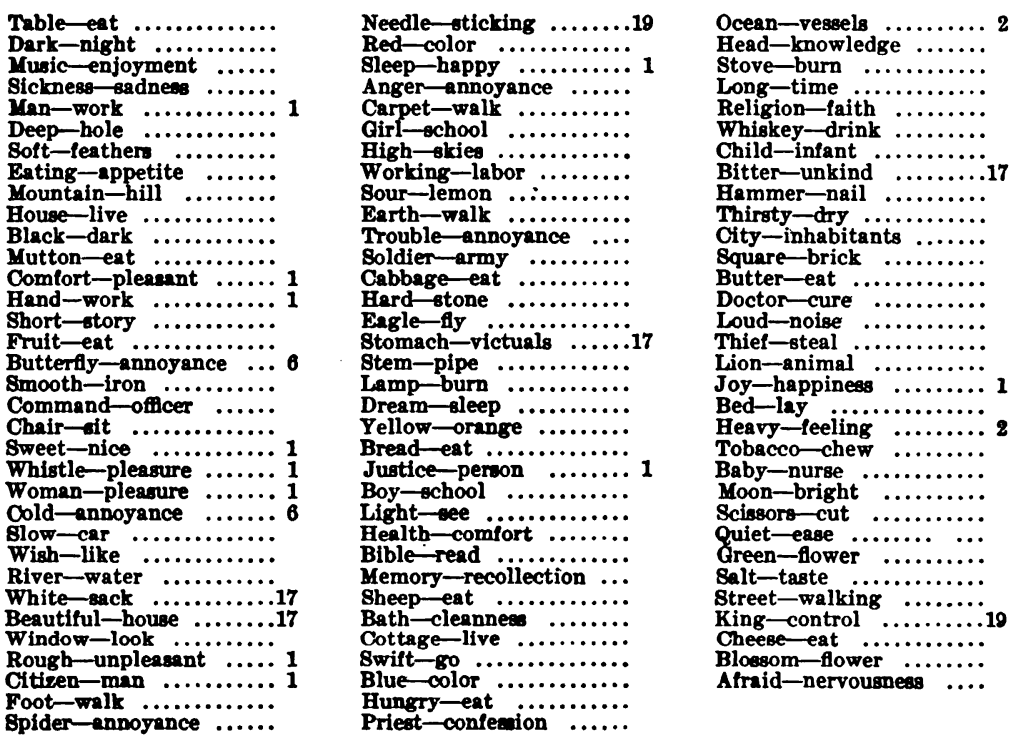

CAse No. 6418.-J. R. Marked deterioration.
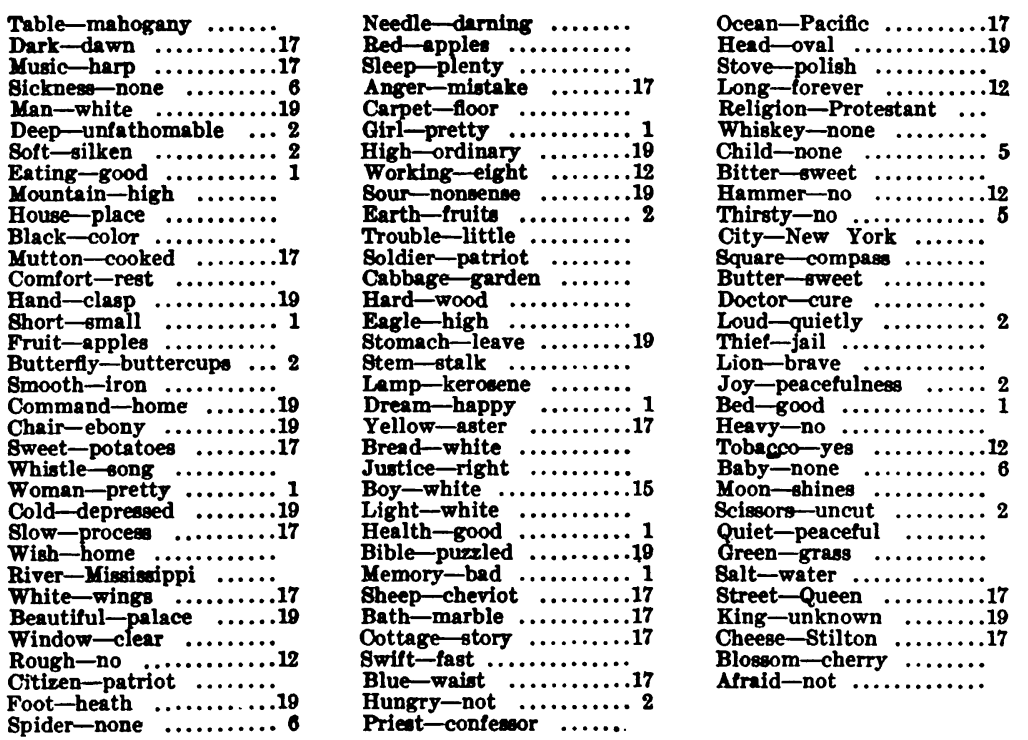
We reproduce in full the record obtained from one of our cases of senile dementia.

Case No. 5788.-E. S.
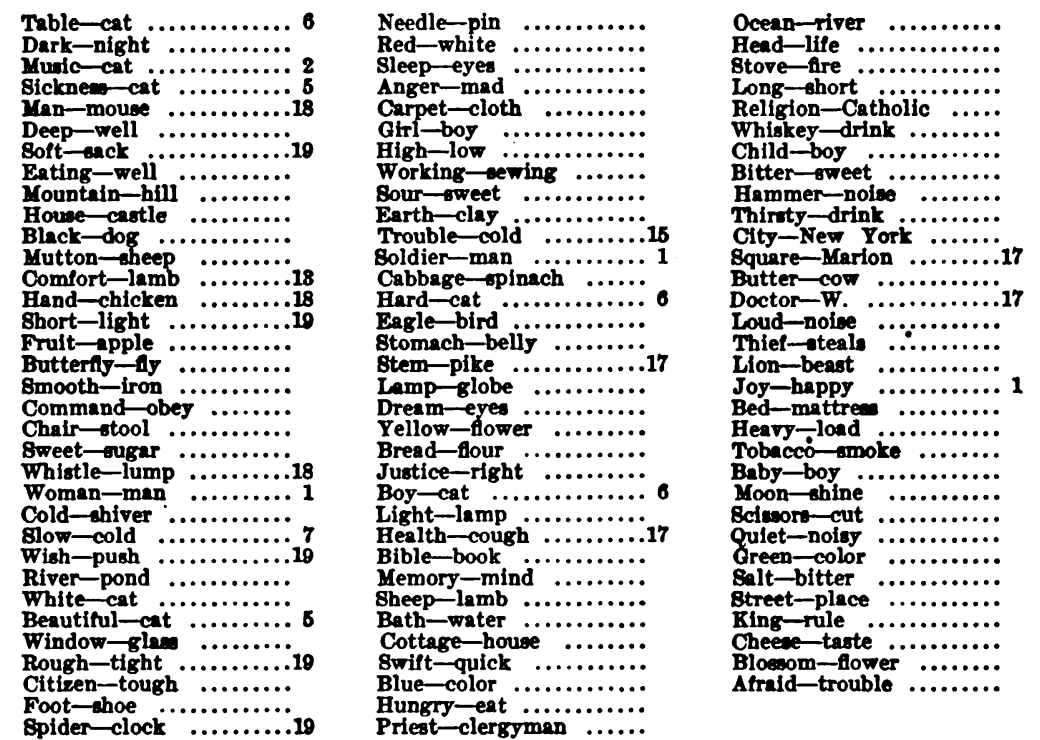

\section{§8. Pathological Reacions from Normal Subjects.}

Mental disorders do not always so manifest themselves as to incapacitate the subject for his work or to necessitate his sequestration in a hospital for the insane. It is, therefore, not surprising that in applying the association test to over a thousand subjects selected at random we have obtained a small number of test records which show various types of abnormal reactions. Among the subjects who furnished such records some are described as eccentric, taciturn, or dull, while others are apparently normal but come of neuropathic stock. A few of them are persons wholly unknown to us.

We reproduce in full several records from the normal series, containing abnormal reactions. 
Consecutrve No. 746.-State hospital attendant. Efficient in his work but is generally regarded to have married very foolishly. Sound reactions; numerous unclassified reactions.
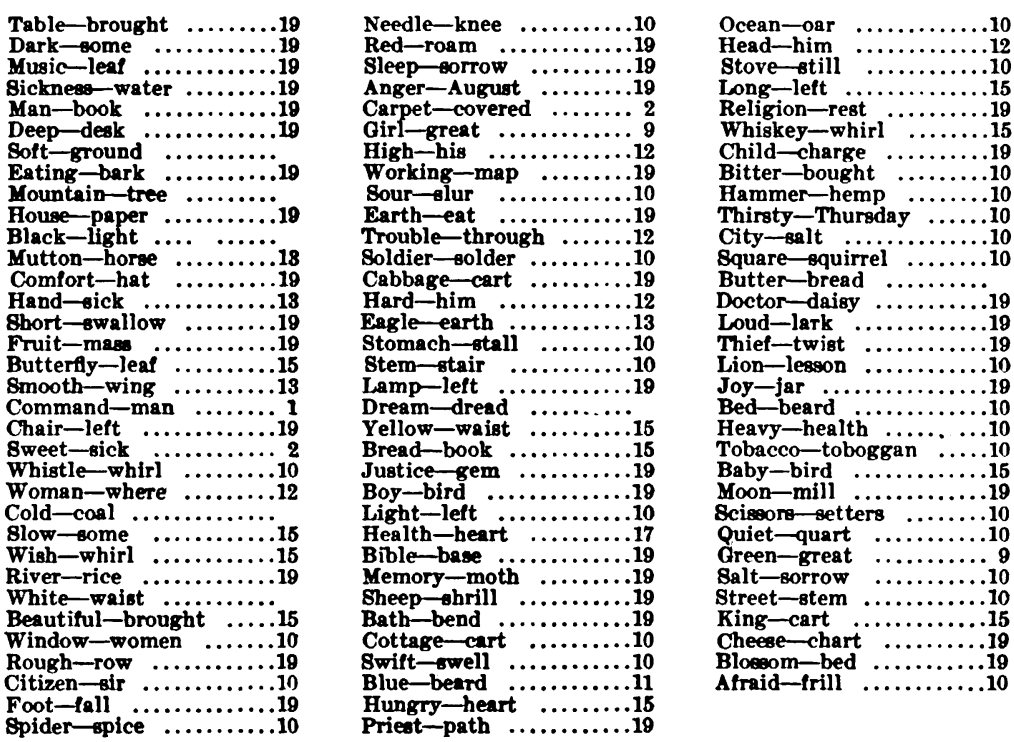

Consecutre No. 220.-Laundryman in State hospital. Nothing abnormal has ever been observed in his case. Numerous perseverations.
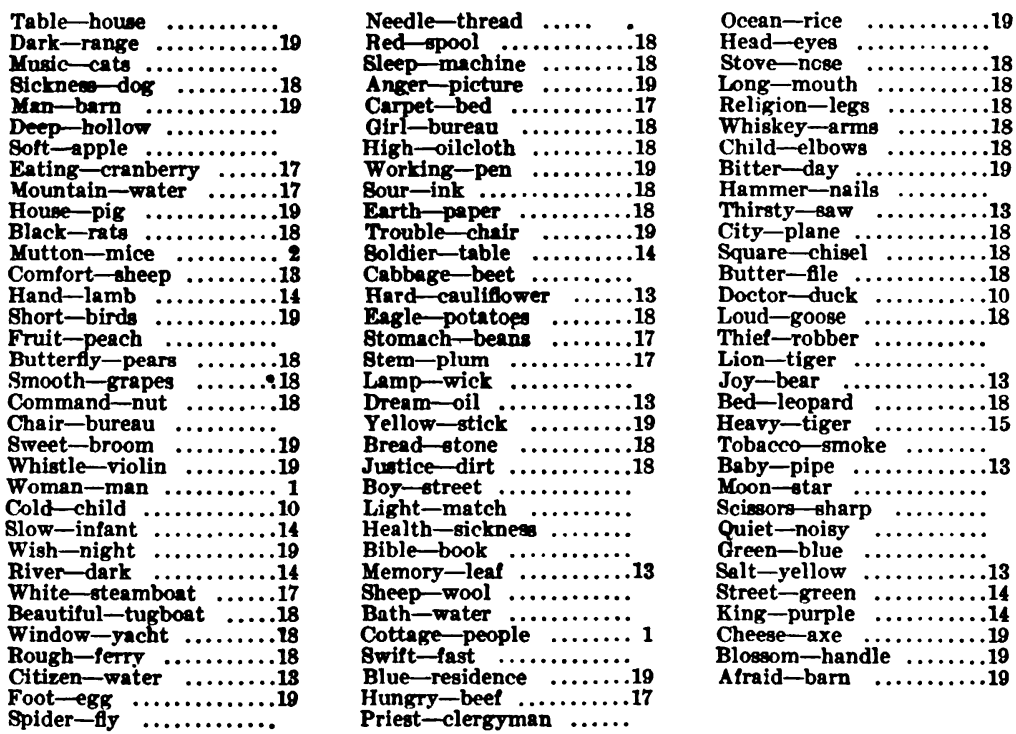
Consecutive No. 255.- State hospital attendant. Efficient, but unusually taciturn and seclusive. Sound reactions.
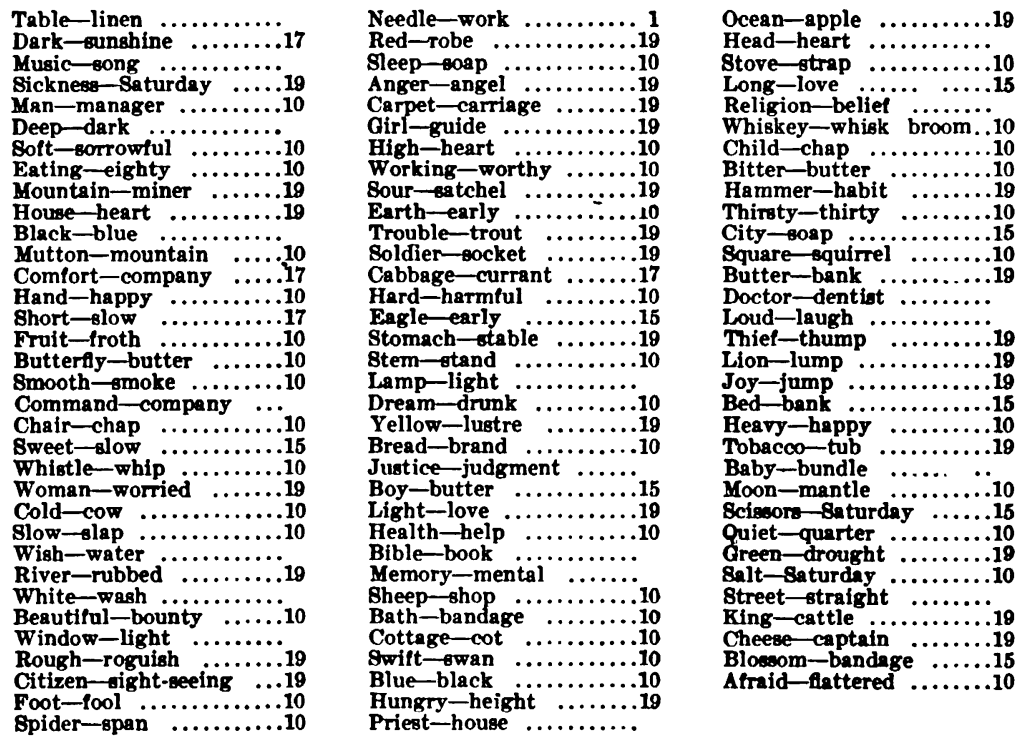

CONSECUTIVE No. 442.- Nothing abnormal has ever been suspected in the case of this subject; mother eccentric; sister insane. Sound reactions.
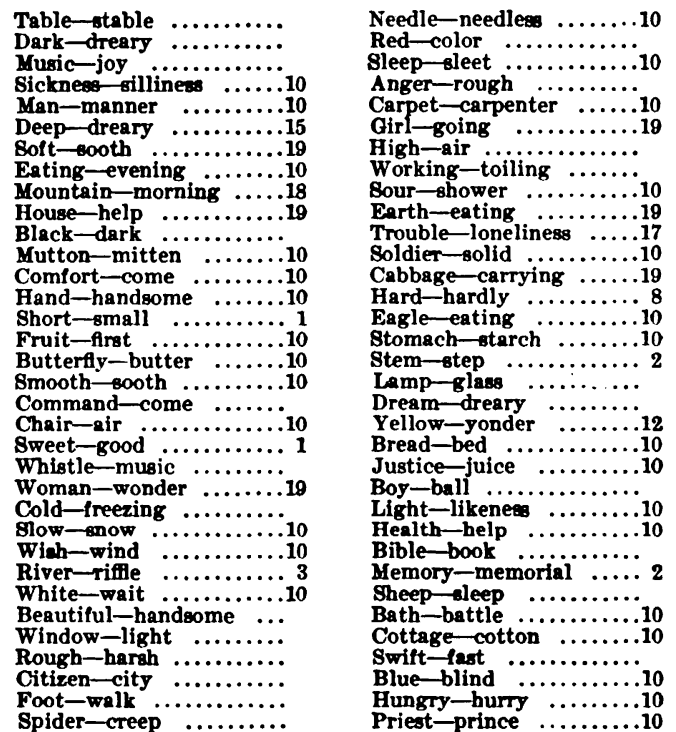

Ocean-over ............12

Head-large

Stove-stone $\ldots \ldots \ldots \ldots 10$

Rong-heavy $\ldots \ldots \ldots \ldots . .19$

Whiskey $\rightarrow$ trong $\ldots . . . . .$.

Whiskey otrong $\ldots . . . \cdots$.

Bitter-butter $\ldots \ldots \ldots \ldots \ldots 10$

Hammer -hard $\ldots . . . \cdots \cdots$

Thirsty-thrifty $\ldots . . \cdots \cdots \cdot 10$

Thirsty-thrifty.$\ldots \ldots \ldots \ldots 10$

Square $\rightarrow$ quirrel $\ldots \ldots \ldots \ldots 10$

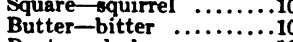

Doctor-dark ............10

Doud-noisy

Thief $\rightarrow$ stegling..........

Lion-esting $\ldots . . . . .$.

Joy $\rightarrow$ joyous $\ldots \ldots \ldots \ldots$,

Bed-sleep

Heary-weighttui...........

Tobacco-cocos

Baby-boys ..............

Moon-moo $\ldots \ldots \ldots \ldots \ldots$ s

Scissors-succeseors .....10

Quiet eany ..............

Green-grass $\cdots \cdots \cdots \cdots$

Street-oteep ............10

King-kingdom ….......

Cheese- -queere ........10

Afraid-Africa ...........10 
[Oct.

Consecutive No. 3I4.-School teacher. Efficient; described as very silent. Unclassified reactions, due mostly to distraction.
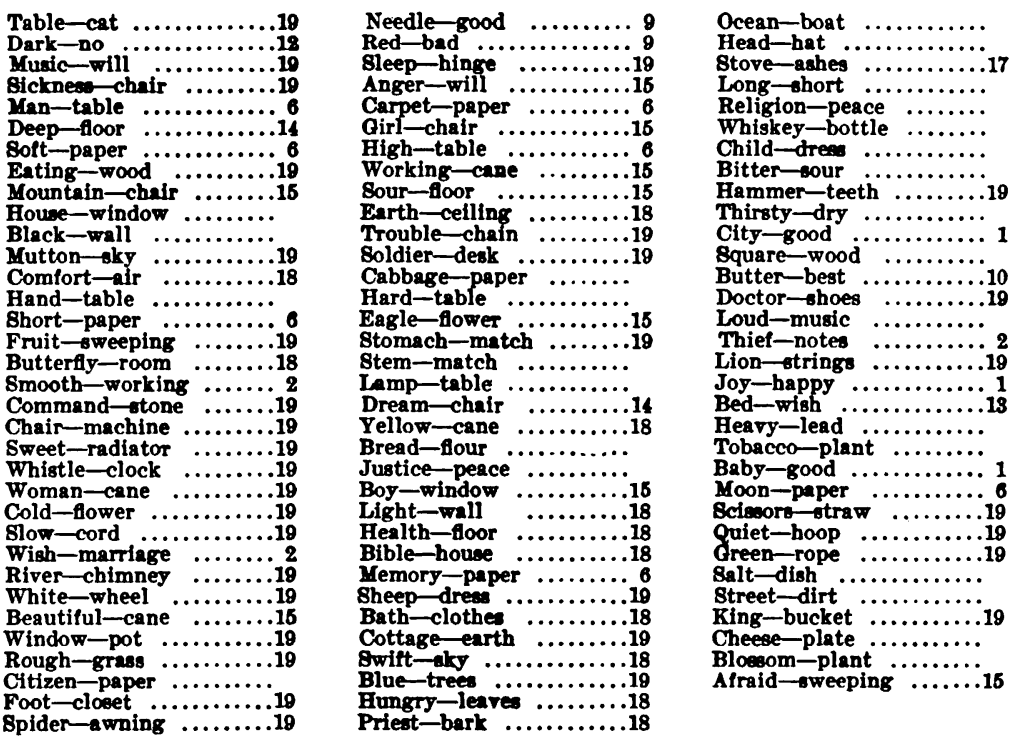

Consecutive No. 216.-State hospital attendant. Incompetent, dull. Numerous non-specific reactions.
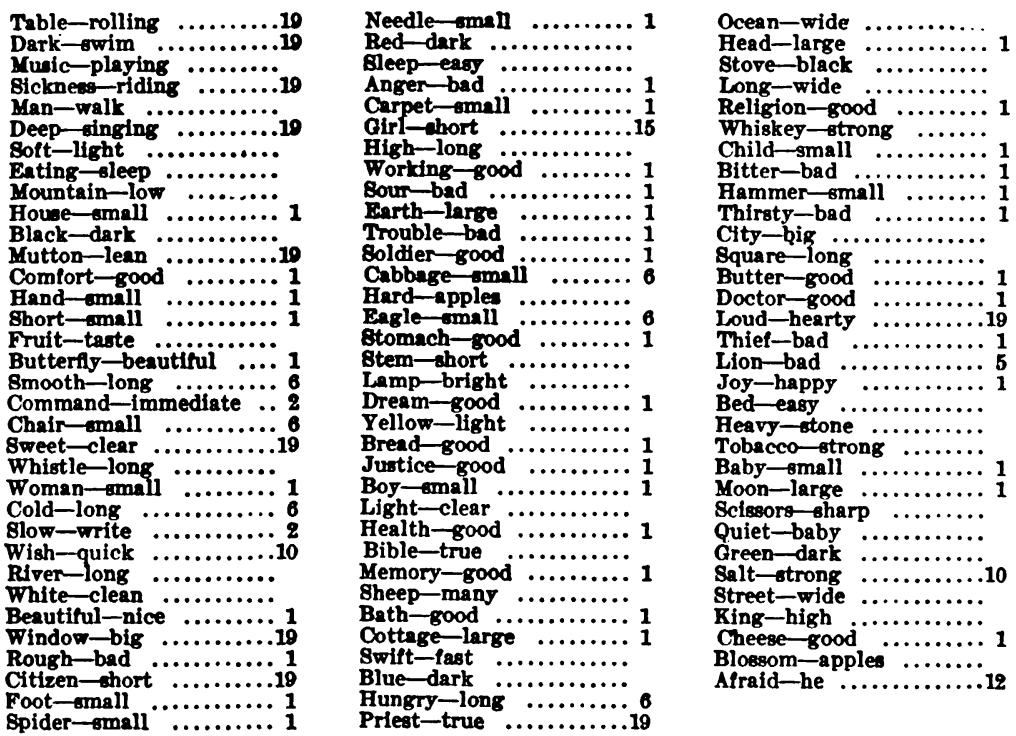
ConsecutTE No. 318.-School boy. Non-specific reactions.
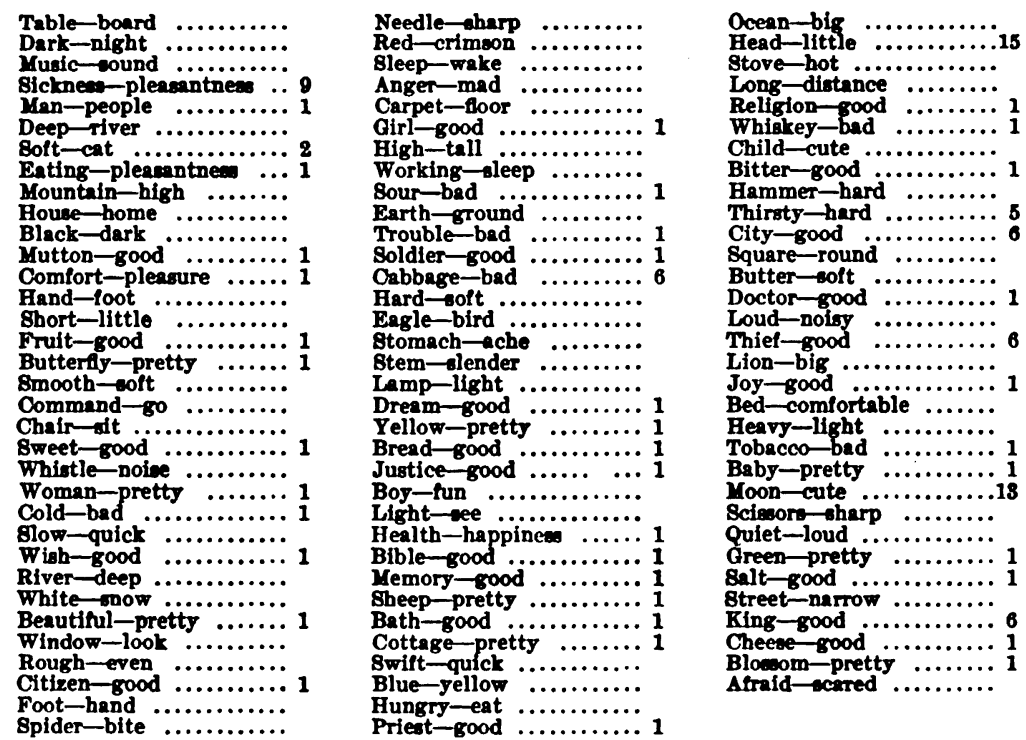

Consecutrve No. 234-School boy. Non-specific reactions.
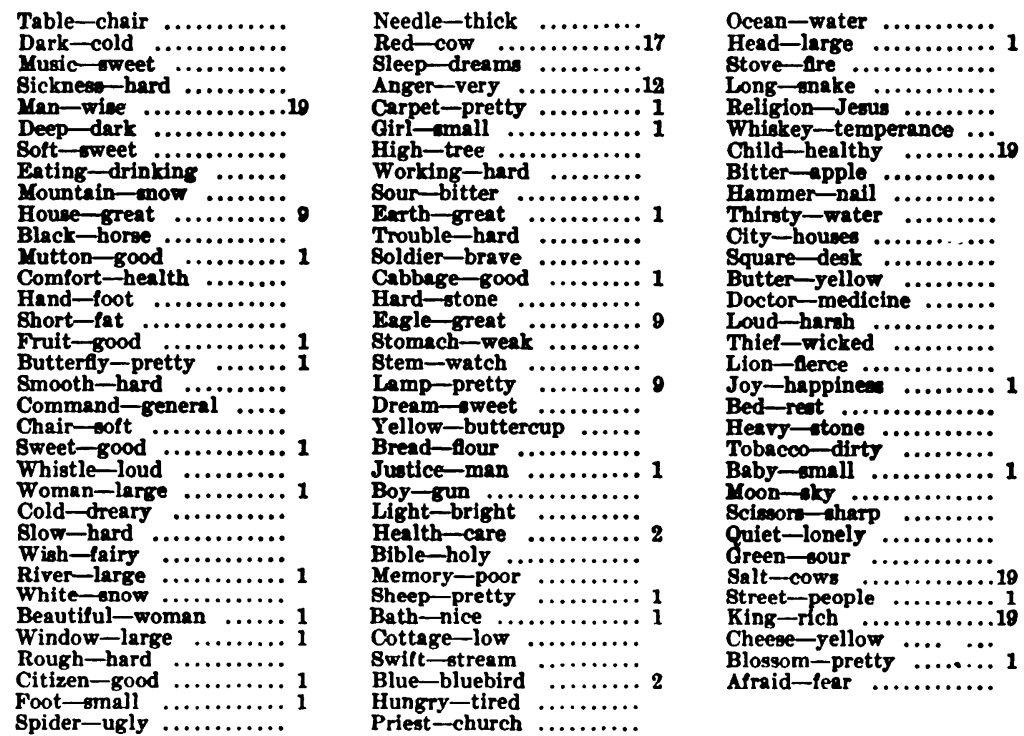

spider-ugly ............

Priest-church...$\ldots \ldots$. 
Consecutrve No. 809.-Lawyer. 28 individual reactions, of which 14 are classed as normal; to are unclassified, most of which are also obviously normal.
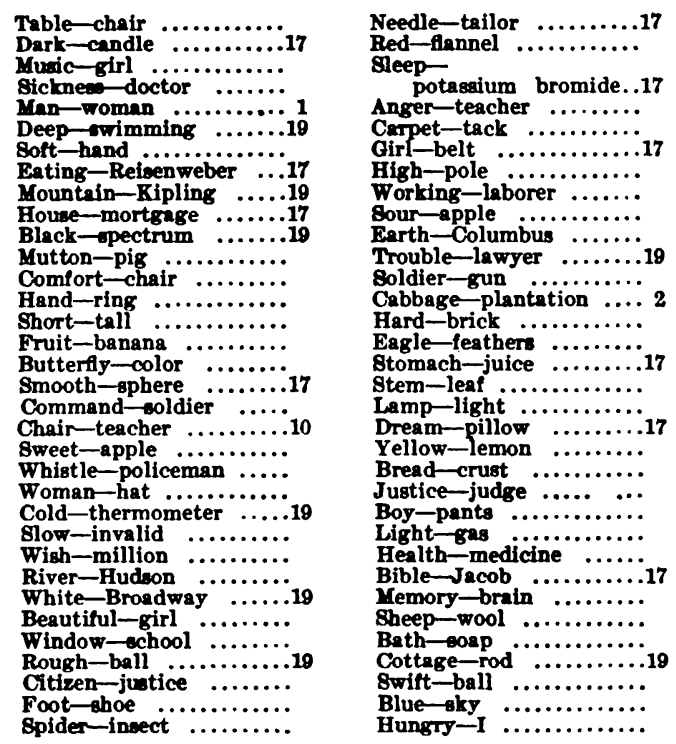

Priest-surplice $\ldots \ldots \ldots$ Ocean-ship Head-hair $\ldots \ldots \ldots \ldots \ldots$. Stove-shovel $\ldots \ldots \ldots \ldots .17$ Long-pole $\ldots \ldots \ldots \ldots . . .10$ Religion-Abraham $\ldots \ldots 19$ Child-baby ............. Bitter-pepper Hammer-nail $\ldots \ldots \ldots \ldots$ Thirsty-lemonade ...... City-Manhattan .......17 Square-Washington .... Butter-malt Doctor-nurse Loud-hammer ......... Thief-jewelry $\ldots \ldots \ldots$ Lion-Androcles $\ldots \ldots \ldots . .10$ Boy-automobile $\ldots \ldots \ldots$. Heavy-Flannigan ........19 Tobacco-pipe $\ldots \ldots \ldots . .$. Baby-wife $\cdots \cdots \cdots \cdots$ Sciseors Ouiet-demure $\cdots \cdots \cdots \cdots$ Quiet-demure $\cdots \cdots \cdots$ Salt-cellar Street-Wall …......... King-Edward Cheese-Roquetort Blosem-fleld Atraid-burglar.

Spider-insect $. . . \ldots \ldots .$.

\section{§ 9. Number of Different Words Given as Reactions.}

It has been suggested by Fuhrmann ${ }^{2}$ that the number of different words given in response to one hundred selected stimulus words may be used as " a fairly reliable measure of the intelligence and degree of education of a patient." The test according to Fuhrmann is applied twice in every case, the interval between the two sittings being at least four weeks. "In very intelligent and well educated persons every 100 stimulus words almost always evokes in the first test 95-100 different associations; in the less intelligent and in the feeble-minded the same associations are more frequently repeated. In the second test with the same stimulus words-which is really much more important than the first, since even persons or inferior intelligence may reach higher numbers in the first test-the difference in the wealth of the stock of representations becomes plainly evident:

'Diagnostik und Prognostik der Geisteskrankheiten, p. 93. Leipzig, 1903. 
the man of intelligence will not need to draw on the associations which he gave in the first test, but will produce new ones; the feeble-minded subject will, on the contrary, repeat to a greater or lesser extent the associations of the first test." "In general the associational capacity of an adult person may be taken to be from 80 per cent to 90 per cent. Should the number sink below 70 per cent the suspicion of a pathological condition must then arise; and the higher the subject's degree of education the stronger is this suspicion. In the case of an associational capacity of 60 per cent or less no doubt of its pathological significance can remain any longer."

Our results are not strictly comparable with Fuhrmann's, because we have obtained but one test record from each subject; it may be said, however, that the results of a single test in each case do not show any considerable differences, corresponding to education or age, in the variety of responses. Further, dementing psychoses, with the exception of epilepsy, show on the whole no diminution in the number of different reactions, although in individual cases this number falls considerably below the general average; and in such cases the diminution may be dependent upon stereotypy or perseveration, and not necessarily upon reduction in the stock of representations.

It would appear from our results that pathological mental states are apt to manifest themselves by a tendency to give reactions belonging to types of inferior values rather than by diminished variety of responses.

We show in Table VI. the numbers of different responses given by our groups of normal and insane subjects, expressed in figures giving for each group the median and the average.

TABLE VI.

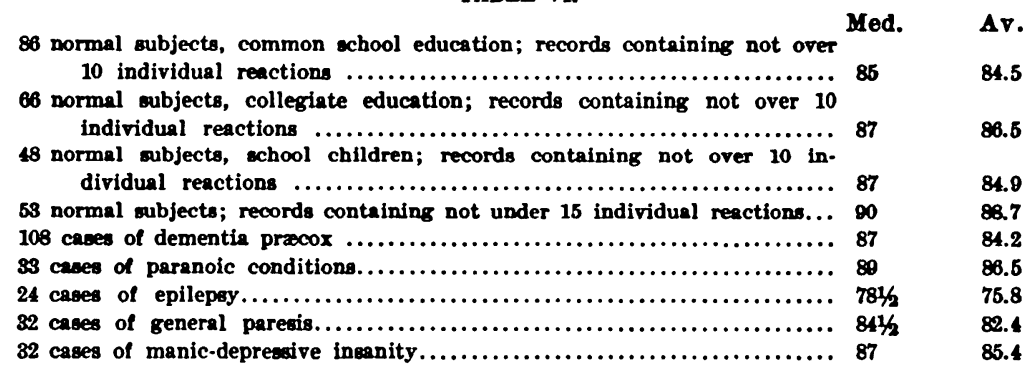




\section{§ io. Co-operation of the Subject.}

In our work with insane subjects we encountered many cases in which we were unable to obtain satisfactory test records owing to lack of proper co-operation. Some subjects seemed to be either too confused or too demented to be capable of understanding and following the instructions given them. Others were for one reason or another unwilling to co-operate. It is important to distinguish inability from unwillingness to co-operate, since the former indicates in itself an abnormal state of the mind, while the latter is quite often shown by normal persons.

A subject may co-operate to the extent of giving a single word in response to each stimulus word, and yet fail to co-operate in some other particulars.

$\mathrm{He}$ may, instead of giving the first word suggested to him by the stimulus, suppress the first word more or less systematically, and give some other word which may seem to him more appropriate. This probably occurs very often, but does not seem to render the results less serviceable for our purpose.

Further, a subject may react by words related not to the stimulus words, but to each other, thus simulating perseveration; or he may react by naming objects within reach of the senses, thus appearing to be distracted; or he may give only sound reactions.

There is, in fact, no type of pathological reactions which a normal person may not be able to produce more or less readily at will, though in the case of incoherent reactions considerable mental effort may be required, and the end may be attained only by regularly rejecting the first and some subsequent words which are suggested by the stimulus.

In view of these considerations we are led to conclude that the association test, as applied by our method, could not be relied upon as a means of detecting simulation of insanity in malingerers, criminals, and the like.

\section{$\S$ II. Sum Mary.}

The normal range of reaction in response to any of our stimulus words is largely confined within narrow limits.

The frequency tables compiled from test records given by one thousand normal subjects comprise over ninety per cent of the normal range in the average case. 
With the aid of the frequency tables and the appendix normal reactions, with a very few exceptions, can be sharply distinguished from pathological ones.

The separation of pathological reactions from normal ones simplifies the task of their analysis, and makes possible the application of a classification based on objective criteria.

By the application of the association test, according to the method here proposed, no sharp distinction can be drawn between mental health and mental disease; a large collection of material shows a gradual and not an abrupt transition from the normal state to pathological states.

In dementia præcox, some paranoic conditions, manic-depressive insanity, general paresis, and epileptic dementia the test reveals some characteristic, though not pathognomonic, associational tendencies.

\section{ACKNOWLEDGMENTS.}

It is with pleasure that we acknowledge our indebtedness to the many persons who have assisted us in collecting the data for this work.

About two hundred tests upon normal subjects were made for us by the following persons: Dr. Frederic Lyman Wells, Dr. Jennie A. Dean, Miss Lillian Rosanoff, and Miss Madeleine Wehle.

Dr. O. M. Dewing, the late superintendent of the Long Island State Hospital, and Dr. Chas. W. Pilgrim, superintendent of the Hudson River State Hospital, have assisted the work by kindly permitting the test to be made upon employees of these institutions, and we are especially indebted to Dr. F. W. Parsons for personal assistance in securing the co-operation of many subjects.

Professor R. S. Woodworth, of Columbia University, extended to us the courtesy of the Psychological Laboratory during several weeks of the summer session of 1909, and gave us much assistance in obtaining interviews with students; we received assistance also from several of the instructors of Teachers' College, especially Mr. Wm. H. Noyes. 
[Oct.

We are indebted to Mr. F. C. Lewis and Mr. W. E. Stark, of the Ethical Culture School, New York, for permitting us to make the test upon the pupils of that school.

In the work of compiling the tables we have been assisted by Dr. N. W. Bartram, Dr. Jennie A. Dean, Mrs. H. M. Kent, and others.

We wish, finally, to express our thanks to Dr. Wm. Austin Macy, superintendent of this hospital, to whom we are indebted for the opportınity of undertaking this work.

\section{APPENDIX TO THE FREQUENCY TABLES}

\section{General Rules.}

I. Any word combination which is to be found in the frequency tables, but only in the reverse order from that in which it occurs in a test record under consideration, is to be classed as a normal reaction.

2. Any reaction word which is a synonym or an antonym of the corresponding stimulus word is to be classed as normal.

\section{TABLE}

Any food or meal.

Any room or apartment.

Any article of table linen, china, silver, or furnishings.

Word designating any special variety of tables.

Any word pertaining to appetite.

\section{DARK}

Any source of illumination.

Any enclosure from which light is wholly or in a large measure excluded. Word referring to physiological pigmentation of tissues exposed to view. Any division of the diurnal cycle.

Any color or coloring material.

Anything which obscures light.

8. MUsic

Any musical instrument.

Name of any composer or musician.

Special or general name of any musical composition.

Term designating rhythm, tempo, loudness, or pitch.

Name of any dance.

Term expressing subjective effect of music. 


\section{SICKNESS}

Term designating any disease, symptom, injury, or physiological function. Any cause of disease.

Any means or measure of treatment of disease.

Any anatomical organ or region.

Word denoting mode of termination, results, consequences, or indirect effects of disease.

Any term of prognostic import.

Common or proper name of any person.

\section{MAN}

Word denoting or implying age of a person.

Any of the well-known male sexual characteristics.

Occupation or profession more or less peculiarly masculine.

Word pertaining to familial relationships or domestic organization.

Word pertaining to sexual relationships; any word denoting the opposite sex.

The proper name of any male person.

Any article of male apparel.

\section{DEEPP}

Any vessel or container.

Any natural or artificial body of water.

Any depression of surface.

Any object naturally situated or often artificially placed at a comparatively great distance below the surface.

Any act of progress from surface to depth.

Any article of food

$$
\text { 7. SOFT }
$$

Any fabric.

\section{EATING}

Any article of table linen, china, or silver.

Any organ of digestion; any function of nutrition.

Any article of food; any meal.

Any private or public eating place.

Word denoting taste.

\section{MoUNTAIN}

Name of any mountain, mountain range, or mountainous country.

Word pertaining to shape, geological composition, fauna, or flora of mountains or mountainous regions.

Any term of physical geography. 


\section{HOUSE}

Any place of house location.

Any part of a house.

Any material used in the construction of a house.

Any part of the process of construction of a house.

Laborer or mechanic having to do with the construction of a house.

Any commercial term pertaining to ownership, taxes, murtgages, sale, renting, or occupancy of a house.

Any article of furniture.

\section{BLACK}

Any object or substance that is always or often black or dark in color. Any color.

Word denoting limitation or obscuration of light.

Any word clearly related to the word Black used as a proper name.

\section{MUTTON}

Any article of food; any meal.

Any animal, or class or group of animals, whose meat is used for human consumption as food.

Any article of table linen, china, silver; any cooking utensil.

Word designating any person engaged in the preparation of meats for consumption.

Word denoting any process employed in the preparation of meats for consumption.

\section{COMFORT}

Any agreeable or disagreeable subjective state.

Any object, act, or condition that contributes to comfort or produces discomfort.

\section{HAND}

Any simple function of the hand; work requiring special manipulation. Word denoting skill or any degree of skill.

Any part or any tissue of the body.

\section{SHORT}

Any word involving the concept of duration. Common or proper name of any person.

Any word denoting shape, relative or absolute dimension, or distance. Any object in which characteristically one dimension exceeds any other. 


\section{FRUIT}

Any article of food; any meal.

Any process employed in the cultivation of fruits or in their preparation for consumption.

Word designating any person engaged in the cultivation of fruits or in their preparation for consumption.

Any article of table linen, china, or silver.

\section{BUTTERFLY}

Any bird, worm, or insect.

Any flower.

Any color.

\section{SMOOTH}

Any object possessing a smooth surface as a characteristic feature. Any fabric.

\section{COMMAND}

Word denoting any means of influence of one mind upon another intended to produce acquiescence.

Word denoting or implying acquiescence or lack of it.

Term applied to any commanding officer or to any person in authority.

\section{CHAIR}

Any article of furniture.

Any room or apartment.

\section{SWEET}

Any substance having a sweet taste.

Common or proper name of a child or woman.

\section{WHISTLE}

Any instrument or any animal producing a shrill musical sound.

\section{WOMAN}

Word denoting or implying age of a person.

Any of the well-known female sexual characteristics.

Occupation or profession more or less peculiarly feminine.

Word pertaining to familial relationships or domestic organization.

Word pertaining to sexual relationships; any word denoting the opposite sex.

Name of any female person.

Any article of female apparel. 


\section{COLD}

Name of any location characterized by low temperature.

Any illness or symptom which may be caused by exposure to cold.

Any division of the annual cycle.

Any food that is always or often served cold.

Any means or measure of protection against cold.

Any state of the natural elements causing a sensation of cold.

Word denoting subjective characterization of or reaction to cold.

\section{5. sLow}

Any means or manner of locomotion.

Any word involving the concept of rate of progress with reference either to time or to intensity of action.

Common or proper name of any person.

\section{Wrsh}

Word implying fulfilment of a wish either by achievement or through acquiescence.

Word implying non-fulfillment of a wish.

Word denoting any state of longing or anticipation.

Word denoting any state free from longing or anticipation.

Word denoting a prayer or request.

Word denoting a state of happiness.

\section{RIVER}

Any body of water.

Any part of a river.

Any plant or animal living in rivers.

Any term of physical geography.

Any vessel or contrivance for navigation.

\section{WHITE}

Any object or substance that is always or often white or very light in color. Any color.

Any word clearly related to the word White used as a proper name.

\section{BEAUTIFUL}

Any word denoting aesthetic pleasure.

Name or any female person.

Any product of the fine arts or of decorative handicraft.

Any decorative plant or flower.

Any article of attire.

Natural scenery.

Any division of the diurnal cycle. 


\section{WINDOW}

Any word pertaining to illumination.

Word pertaining to movements of air.

Any attachment to a window for the control of transmission of light or air. Any building or apartment.

\section{ROUGH}

Any object or substance which is characteristically rough to the touch. Word denoting or implying irregularity of surface.

Any skin lesion which may impart to the skin the quality of roughness.

Any word implying carelessness, lack of consideration, or crudeness; any word used to designate action or conduct which may be characterized as careless, inconsiderate, or crude.

\section{CITIZEN}

Any word pertaining to political organization, or to factors either favorable or unfavorable to it.

Any term or proper name of political geography.

Common or proper name of any male person.

\section{FOOT}

Any means or manner of locomotion involving the use of the feet. Any part or any tissue of the animal body.

Any article of foot-wear.

Any way constructed or used for walking.

Any unit of linear measure.

\section{SPIDER}

Word employed to designate subjective characterization of or reaction to an object of dislike.

Any insect.

Word pertaining to the characteristic habits of spiders, with reference either to location and construction of nest, or to manner of catching prey.

\section{NEEDLE}

Any material used in making clothes.

Any special sewing operation; any occupation in which sewing constitutes part of the work.

Any special kind of needles.

Any instrument which is used in connection with a needle in any operation, or of which a needle forms a part. 
86. RED

Word which may be used to express subjective characterization of the red color.

Any object or substance which is always or often red in color.

Anything which is by convention or common usage connected with the red color.

Any organ, tissue, or lesion, exposed to view, which may have a red color imparted to it by the hlood or by physiological pigment.

Any color or coloring material.

Any word implying light through incandescence.

\section{SLEEL"}

Word denoting somnolence or a state of lowered consciousness; anything which is a cause of somnolence or of lowered consciousness; anything which induces a desire to sleep.

Word denoting a state of active consciousness or a transition from lowered to more active consciousness.

Any division of the diurnal cycle.

Any word more or less commonly used to characterize sleep in any way.

Any article of bedding, bed-linen, or night-clothes.

Any article of furniture used for sitting or lying.

\section{ANGER}

Any affective state; any common demonstration of emotion.

Any common cause or provocation of anger.

Action or conduct caused by anger; word used to characterize such action or conduct.

\section{CARPET}

Any material of which carpets are made.

Any article of house furniture, hangings, or decorations.

Word denoting home, house, or any part of a house.

Word pertaining to the manufacture or care of carpets, or denoting a person engaged in the manufacture, sale, or care of carpets.

Any country especially noted for the manufacture of carpets or rugs.

Any color.

\section{GIRL}

Word denoting or implying age of a person.

Any of the well-known female sexual characteristics.

Occupation or profession more or less peculiarly feminine.

Word pertaining to familial relationships or domestic organization.

Word pertaining to sexual relationships; any word denoting the opposite sex.

Name of any female person.

Any part of a person's body.

Any article of female apparel. 


\section{HIGF}

Any word denoting or implying skill, training, achievement, or position.

Any word denoting or implying valuation.

Any architectural structure.

Any object of which the vertical dimension characteristically exceeds any other.

Any act of progress from a lower to a higher level.

Name of any mountain or mountain range.

Anything characteristically situated at a high level.

Anything characteristically variable in height.

\section{WORKING}

Any occupation, profession, art, or labor. Direct results or consequences of work.

Any place of employment.

Rest, recreation, inaction, or disinclination to work.

Word denoting energy, material, capital, equipment.

\section{SOUR}

Any substance or object which is always or often sour in taste. Any word denoting a taste or flavor quality.

\section{FA RTH}

Any substance which enters into the composition of soil.

Word pertaining to the utilization or cultivation of natural resources; any product of agriculture.

Any term of physical geography, geology, mineralogy, meteorology, or astronomy.

\section{TROUBLF}

Any affective state.

Any general cause of active emotional states.

Any common manifestation of emotion.

Word denoting or implying defeat.

Word denoting or implying caution or lack of it.

Any task.

\section{SOLDIER}

Word pertaining to military organization.

Word pertaining to any military operation.

Word pertaining to military discipline or to military decoration.

Any article of military or naval equipment or attire.

Common or proper name of any male person.

Name of any country.

Word pertaining to political organization. 


\section{CABBAGE}

Any article of food; any meal.

Any article of table linen, china, silver; any cooking utensil.

Any process of cooking.

Word used to designate any person engaged in the cultivation of cabbages or in their preparation for consumption.

\section{HARU}

Any solid article of food.

Word denoting or implying impact.

Any task or labor.

Any substance which is hard or unyielding.

Any agency or process by which a substance is solidified or hardened.

Any article of furniture used for sitting or lying.

Any trait of disposition characterized by lack of readiness to yield or lack of consideration for others.

49. FAGLE

Any bird.

Any piece of currency.

Anything in connection with which the word eagle is used in a symbolic sense.

\section{STOMACE}

Any anatomical organ or region.

Any article of food; any meal.

Word pertaining to ingestion and assimilation of food.

Term denoting health or disease; any medicament.

\section{STERM}

Any object which has a stem.

Any part of a plant.

Any object which is long, slender, and more or less rigid.

62. LAMP

Any means or source of illumination.

Word denoting or implying illumination.

\section{DREAM}

Any product of imagination.

Any psychical phenomenon; any part of the psychical organ.

Word denoting or implying unreality or uselessness.

Word denoting or implying mystery or occultism.

Any division of the diurnal cycle.

Any article of bedding, bed-linen or night-clothes.

Any article of furniture used for sitting or lying.

Any narcotic substance. 


\section{YALLOW}

Word which may be used to denote subjective characterization of the yellow color.

Any object or substance which is always or often yellow in color.

Any color or coloring material.

\section{BREAD}

Any article of food; any meal.

Any article of table linen, china, or silver; any cooking utensil.

Any private or public eating place.

Word pertaining to ingestion and assimilation of food.

Any ceremony in connection with which bread is used.

\section{JUSTICE}

Any word implying crime or tendency to crime, legal trial, retribution or lack of it, or repentance.

Any officer of the law.

Word pertaining to judiciary organization.

Word denoting any kind of ethical relationship.

Any deity.

The name of any justice or judge.

Any function of a judicial authority.

Any word denoting or implying equality.

\section{BOY}

Word denoting or implying age of a person.

Word pertaining to familial relationships or domestic organization.

Word pertaining to sexual relationships; any word denoting the opposite sex.

Common or proper name of any male person.

Any part of a person's body.

Any article of male apparel.

Any common boys' toy or game.

Word pertaining to educational organization.

\section{HGHT}

Any source, apparatus, or means of illumination.

Any color or coloring material.

Word implying light through incandescence.

Any term of optics; any optical phenomenon.

Any object or substance which is characteristically light in weight. 


\section{HEALTH}

Any emotion; any common manifestation of emotion.

Any disease or symptom.

Word pertaining to prevention or treatment of disease.

Word pertaining to any normal bodily function.

Word pertaining to the preservation of health.

Word denoting or implying a state of health.

Any athletic sport or form of exercise.

Any anatomical organ or region.

\section{BIBLE}

Name of any personage mentioned in the Bible.

Any religion or religious denomination.

Any name or attribute employed in reference to the Deity.

Any article or act of religious ritual.

Word denoting or implying belief, disbelief, or doubt.

Any term of theology.

\section{MEMORY}

Word pertaining to operations, faculties, endowment, training, or conditior of the mind.

Word denoting any degree of accuracy.

Word denoting the cranium; any part of the psychical organ.

Word pertaining to the past.

Any word implying transiency.

Any subject of study involving the exercise of memory.

Any method or means for the reinforcement of memory.

Any of the senses.

Word denoting retention.

\section{SHEREP}

Any animal raised or hunted for clothing material, for food, or for its services as a beast of burden.

Any product manufactured from the skin or wool of sheep.

Any of the more or less distinctive characteristics of sheep.

Any food product derived from sheep.

\section{BATH}

Word denoting or implying an effect of bathing on the body. Any body of water.

Any kind of bath; any part of bath, lavatory, or toilet equipment. Any material of which a bathing equipment is largely made. Word denoting a state of partial or complete undress. Any beach or bathing resort.

Any aquatic feat of gymnastics. 


\section{COTTAGE}

Word pertaining to landscape gardening.

Any place of cottage location.

Any part of a house; any color.

Any material used in the construction of a cottage.

Any laborer or mechanic having to do with the construction of a cottage.

Any part of the process of construction of a cottage.

Any commercial term pertaining to ownership, taxes, mortgages, sale, renting, or occupancy of a cottage.

Any article of furniture.

\section{SWIFT}

Any means or manner of locomotion.

Word denoting or implying motion or rate of motion.

Any animal or familiar object characterized by rapid locomotion.

Any word clearly related to the word Swift used as a proper name.

\section{BLUE}

Word which may be used to express subjective characterization of the blue color.

Any object or substance which is always or often blue in color.

Anything which is by convention or common usage connected with the blue color.

Any organ, tissue, or lesion, exposed to view, which may have a blue color imparted to it by the blood or by physiological pigment.

Any color or coloring material.

\section{HUNGRY}

Any animal.

Any article of food; any meal.

Word denoting taste or flavor.

Word denoting or implying privation or torture.

Any article of table linen, china, or silver.

Any private or public eating place.

Any organ of digestion; any function of nutrition.

Word designating any person engaged in the preparation or sale of foods.

\section{Pribst}

Any religion or denomination.

Any article or act of religious ritual.

Any term of theology.

Word denoting or implying sanctity.

Word denoting or implying belief, disbelief, or doubt.

Word pertaining to church organization.

Proper name of any priest.

Any article of clerical attire.

Any profession more or less peculiarly masculine. 
G9. OCEAT

Any body of water.

Any plant or animal living in the ocean.

Any term of physical geography.

Any vessel or contrivance for navigation.

Word pertaining to navigation; any nautical term.

Common or proper name of any place bordering on the ocean.

Any aquatic feat of gymnastics.

\section{HEAD}

Any organization which has a person occupying the highest office. Word denoting or implying the highest office of any organization. Any intellectual faculty, quality, or operation.

Any part of the head.

Any pathological condition affecting the head.

\section{STOVE}

Any part of a stove.

Any kitchen utensil.

Any artificial heating apparatus; any fuel.

Any manner of cooking; any person engaged in cooking food.

Any article of household furniture.

\section{LONG}

Any word involving the concept of duration.

Word denoting shape, relative or absolute dimension, or distance.

Any object in which characteristically one dimension exceeds any other.

\section{RELTGION}

Any religion or denomination; the name of any race or nation.

Any term of theology.

Any branch of metaphysical philosophy.

\section{WHIKEYY}

Any beverage; the name of any brand of whiskey.

Any material of which whiskey is made.

Word denoting taste or flavor.

Any occasion or ceremony commonly associated with the use of alcoholic beverages.

Word denoting a state of lowered consciousness.

Any physiological or pathological effect of alcohol; also any well known indirect effect.

\section{CHIID}

Word denoting or implying age of a person.

Word pertaining to familial relationships or domestic organization.

Name of any person. 
Any part of a person's body.

Any article of a child's apparel.

Any common child's toy or game.

Word pertaining to educational organization.

Any word descriptive of the natural physical or mental make-up of a child, or of the rate or degree of physical or mental development.

Word pertaining to any custom or ceremony connected with the birth or rearing of children.

Any term of obstetrics.

Any word clearly related to the word Child used as a proper name.

\section{BITTER}

Any substance having a bitter, sour, sweet, or salt taste, or a complex taste quality which may be characterized as strong.

Word denoting a taste or flavor quality.

Any organ of taste.

Any word in connection with which the word bitter may be used in the sense of poignant.

\section{HAMMER}

Any tool or weapon.

Any trade involving the use of a hammer.

\section{THIRSTY}

Any beverage.

Any animal.

Word denoting taste or taste quality.

Any part of the upper end of the digestive tract.

Any drinking place; any container of a beverage.

Any fruit; any dessert.

Any food ingredient commonly known to excite thirst.

78. CITY

Name of any division of political geography.

Any architectural structure.

Any part of a city.

Word pertaining to the political organization of a city.

\section{SQUARE}

The name of any city.

The name of any square in a city or town.

Any geometrical figure or part of one.

Any object that is always or often square in shape.

Any device used in the arts for measuring angles, arcs, or distances between points.

Any part of a carpenter's or draughtsman's square.

Any trade involving the use of the square. 


\section{BUTTER}

Any article of food; any meal.

Any article of table linen, china, or silver; any cooking utensil.

Any process of cooking.

\section{DOCTOR}

The name of any physician.

Any medical speciality or practice.

Any medical or surgical procedure.

Any therapeutic remedy or method.

Any organization for the treatment of disease.

Name of any injury or disease.

83. LOUD

Any sound or sound quality.

Any part of the human vocal apparatus.

Any act of vocalization.

Any musical instrument.

Any apparatus for making sound signals.

Word denoting renown or commendation.

\section{THIEF}

Word denoting crime or wrongdoing.

Word denoting any circumstance propitious for theft.

Any common measure for the prevention or punishment of crime.

Any judicial, police, or penal authority.

Any readily portable article of value.

Word denoting renown.

\section{HION}

Word denoting or implying fear.

Any animal.

\section{6. $20 x$}

Word denoting a state, quality, faculty, or function of the mind. Any common manifestation of emotion.

Any occasion, act, or means of recreation or of pleasurable excitement.

\section{BED}

Any article of bedding, bed linen, or night-clothes.

Any article of furniture.

Any living room, apartment, or building.

Any part of a room.

Any division of the diurnal cycle.

Any material of which beds are made.

Word pertaining to sleep or rest. 


\section{HEA V $Y$}

Word denoting or implying weight or lightness.

Any object or substance which characteristically possesses the quality of either great weight or marked lightness.

Any means of support or suspension.

Any fabric; any article of clothing or bedding.

Word denoting something to be carried or transferred.

Any painful emotion.

Word denoting a state of lowered consciousness.

\section{TOBACCO}

The name of any brand or variety of tobacco.

Term denoting any common quality of tobacco.

Any physiological or pathological effect of tobacco.

Any word which expresses subjective characterization of tobacco.

\section{BABY}

Word denoting or implying age or size of a person.

Word pertaining to familial relationships or domestic organization.

Name of any person.

Any part of a person's body.

Any article of a child's apparel.

Any common child's toy or game.

Word pertaining to any custom or ceremony connected with the birth or rearing of children.

Any term of obstetrics.

\section{MOON}

Any term of astronomy.

Word denoting or implying illumination or obscuration of light.

Any division of the diurnal cycle.

\section{SCISSORS}

Any operation or handicraft involving the use of scissors.

Any fabric; any article of clothing.

Any metal of which scissors are made.

Any tool for cutting, piercing, or sharpening.

Any operation of cutting, piercing, or sharpening.

\section{QUIET}

Any place where silence usually prevails or is enforced.

Word denoting or implying a state of lowered psychical activity or of psychical inhibition.

Word denoting heightened psychical activity.

Any word pertaining to the emotions. 


\section{GREEN}

Word which may be used to express subjective characterization of the green color.

Any object or substance which is always or often green in color.

Anything which is by convention or common usage connected with the green color.

Any color or coloring material.

Any plant, collection of plants, or part of a plant.

Any word clearly related to the word Green used as a proper name.

\section{SALT}

Any article of food that is usually seasoned with salt; any seasoning; any relish.

Any article of table linen, china, or silver.

Any process of cooking.

Any term of chemistry.

\section{STREET}

Name of any street or city.

Any part of a street.

Any building.

Any manner or means of locomotion commonly employed in traveling through streets.

\section{KING}

Any name of the Deity.

The proper or common name of any ruler of a nation or of a smaller municipality.

Any nation or ccuntry.

Any title of nobility.

Any word clearly related to the word King used as a proper name.

\section{ChEESE}

Any article of food; any meal.

Word denoting any variety of cheese.

Word pertaining to taste, flavor, or odor.

Word pertaining to appetite.

Any article of table linen, china, or silver.

\section{8. вLOssom}

Any plant, collection of plants, or part of a plant.

Any term of botany.

Any division of the annual cycle.

\section{AFKAID}

Any affective state; any common demonstration of emotion.

Any common object of fear.

Word denoting or implying danger, courage; any means of defense or protection against danger. 